\title{
CORRELATION STRUCTURE OF INTERMITTENCY IN THE PARABOLIC ANDERSON MODEL
}

\author{
J. Gärtner \\ Fachbereich Mathematik \\ Technische Universität Berlin \\ Strasse des 17. Juni 136 \\ D-10623 Berlin, Germany.
}

\author{
F. den Hollander \\ Mathematisch Instituut \\ Universiteit Nijmegen \\ Toernooiveld 1 \\ NL-6525ED Nijmegen, The Netherlands.
}

\begin{abstract}
Consider the Cauchy problem $\partial u(x, t) / \partial t=\mathcal{H} u(x, t)\left(x \in \mathbb{Z}^{d}, t \geq 0\right)$ with initial condition $u(x, 0) \equiv 1$ and with $\mathcal{H}$ the Anderson Hamiltonian $\mathcal{H}=\kappa \Delta+\xi$. Here $\Delta$ is the discrete Laplacian, $\kappa \in(0, \infty)$ is a diffusion constant, and $\xi=\left\{\xi(x): x \in \mathbb{Z}^{d}\right\}$ is an i.i.d. random field taking values in $\mathbb{R}$. Gärtner and Molchanov (1990) have shown that if the law of $\xi(0)$ is nondegenerate, then the solution $u$ is asymptotically intermittent. This means that $\lim _{t \rightarrow \infty}\left\langle u^{2}(0, t)\right\rangle /\langle u(0, t)\rangle^{2}=\infty$, where $\langle\cdot\rangle$ denotes expectation w.r.t. $\xi$, and similarly for the higher moments. Qualitatively their result says that, as $t$ increases, the random field $\left\{u(x, t): x \in \mathbb{Z}^{d}\right\}$ develops sparsely distributed high peaks, which give the dominant contribution to the moments as they become sparser and higher.

In the present paper we study the structure of the intermittent peaks for the special case where the law of $\xi(0)$ is (in the vicinity of) the double exponential $\operatorname{Prob}(\xi(0)>s)=\exp \left[-e^{s / \theta}\right](s \in \mathbb{R})$. Here $\theta \in(0, \infty)$ is a parameter that can be thought of as measuring the degree of disorder in the $\xi$-field. Our main result is that, for fixed $x, y \in \mathbb{Z}^{d}$ and $t \rightarrow \infty$, the correlation coefficient of $u(x, t)$ and $u(y, t)$ converges to $\left\|w_{\rho}\right\|_{\ell^{2}}^{-2} \sum_{z \in \mathbb{Z}^{d}} w_{\rho}(x+z) w_{\rho}(y+z)$. In this expression, $\rho=\theta / \kappa$ while $w_{\rho}: \mathbb{Z}^{d} \rightarrow \mathbb{R}^{+}$is given by $w_{\rho}=\left(v_{\rho}\right)^{\otimes d}$ with $v_{\rho}: \mathbb{Z} \rightarrow \mathbb{R}^{+}$the unique centered ground state of the 1-dimensional nonlinear equation $\Delta v+2 \rho v \log v=0$ (ground state means the solution in $\ell^{2}(\mathbb{Z})$ with minimal $l^{2}$-norm). Qualitatively our result says that the high peaks of $u$ have a shape that is a multiple of $w_{\rho}$ relative to the center of the peak.

It will turn out that if the right tail of the law of $\xi(0)$ is thicker (or thinner) than the double exponential, then the correlation coefficient of $u(x, t)$ and $u(y, t)$ converges to $\delta_{x, y}$ (resp. the constant function 1). Thus, the double exponential family is the critical class exhibiting a nondegenerate correlation structure.
\end{abstract}

1991 Mathematics Subject Classification: 60H25, 82C44 (primary), 60F10, 60J15, 60J55 (secondary).

Key words: random media, intermittency, large deviations, variational problem, nonlinear difference equation.

Running title: The parabolic Anderson model.

Date: February 20, 1997. 


\section{Contents}

$\mathbf{0}$ Introduction $\quad \mathbf{3}$

0.1 The parabolic Anderson model ................. 3

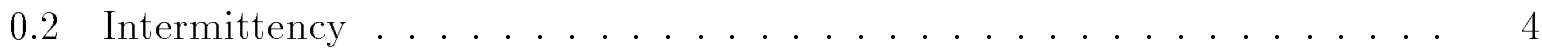

0.3 Correlation structure: $(*)$ and Theorems $1-2 \ldots \ldots \ldots$

0.4 A variational problem: $(* *)$ and Proposition $3 \ldots \ldots 7$

0.5 Asymptotics of the 1 -st and 2 -nd moments: Theorem $3 \ldots \ldots$

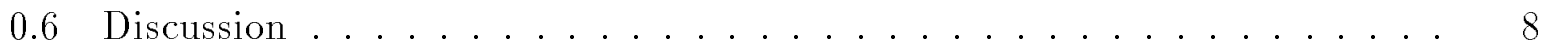

0.7 Numerical study of $(*) \ldots \ldots \ldots \ldots$

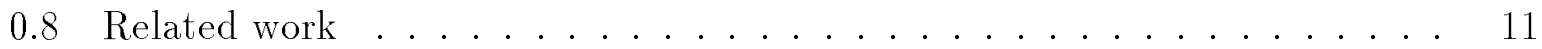

1 Heuristic explanation of Theorem $3 \quad 11$

1.1 Expansion for the 1 -st moment . . . . . . . . . . . . . 11

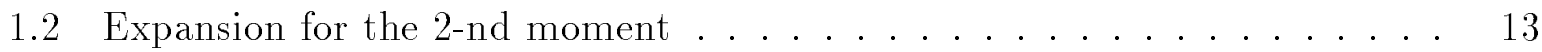

2 Main propositions $\quad \mathbf{1 4}$

2.1 Clumping of the local times: Proposition 4 . . . . . . . . . . . 14

2.2 Centering and truncation of the local times: Proposition 5 . . . . . 15

2.3 Two time scales: Proposition $6 \ldots \ldots \ldots$

2.4 Transformation of the random walk: Proposition $7 \ldots \ldots$. . . . . . . 17

2.5 Separation of the time scales: Proposition $8 \ldots \ldots \ldots$

2.6 Loss of memory: Proof of Proposition 9 . . . . . . . . . . . . 19

2.7 Completion of the proof of Theorem $3 \ldots \ldots \ldots$

3 Proof of Propositions 4-6 21

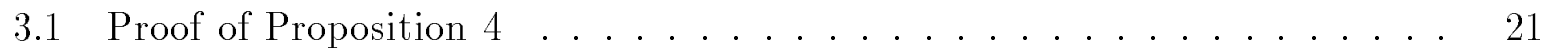

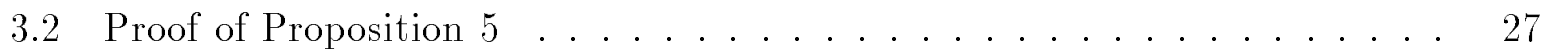

3.3 Proof of Proposition $6 \ldots \ldots \ldots 33$

4 Proof of Propositions 7-9 36

4.1 Proof of Proposition $7 \ldots \ldots \ldots \ldots$

4.2 Proof of Proposition $8 \ldots \ldots \ldots \ldots$

4.3 Proof of Proposition $9 \ldots \ldots \ldots \ldots$

5 Functional analysis $\quad \mathbf{4 2}$

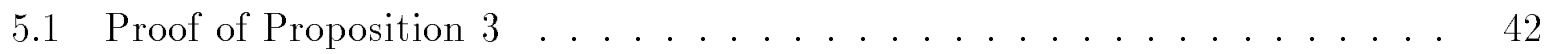

5.1 .1 Analysis of $(* *) \ldots \ldots \ldots \ldots . \ldots \ldots$

5.1 .2 The link between $(* *)$ and $(*) \ldots \ldots \ldots . \ldots 46$

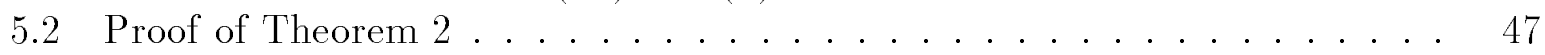

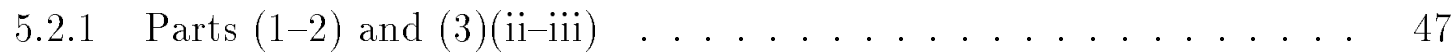

5.2 .2 Parts $(4)$ and $(5) \ldots \ldots \ldots \ldots \ldots \ldots$

5.2 .3 Part $(3)($ iii $) \ldots \ldots \ldots \ldots \ldots \ldots$

5.2 .4 Parts $(6)$ and $(7) \ldots \ldots \ldots \ldots$ 
$5.3 \quad$ Finite approximation of $(* *) \ldots \ldots \ldots \ldots \ldots$

\section{Introduction}

\subsection{The parabolic Anderson model}

Consider the Cauchy problem

$$
\begin{aligned}
& \frac{\partial}{\partial t} u(x, t)=\mathcal{H} u(x, t) \quad\left(x \in \mathbb{Z}^{d}, t \geq 0\right) \\
& u(x, 0) \equiv 1
\end{aligned}
$$

with $\mathcal{H}$ the Anderson Hamiltonian

$$
\mathcal{H}=\kappa \Delta+\xi
$$

Here $\Delta$ is the discrete Laplacian, $\kappa \in(0, \infty)$ is a diffusion constant, and

$$
\xi=\left\{\xi(x): x \in \mathbb{Z}^{d}\right\}
$$

is an i.i.d. random field taking values in $\mathbb{R}$. As an operator, $\mathcal{H}$ only acts on the spatial variable:

$$
\begin{aligned}
(\Delta u)(x, t) & =\sum_{y:|y-x|=1}[u(y, t)-u(x, t)] \\
(\xi u)(x, t) & =\xi(x) u(x, t) .
\end{aligned}
$$

Note that $\mathcal{H}$ has two competing parts:

(1) a diffusive part $\kappa \Delta$, which tends to make $u$ spatially flat;

(2) a multiplicative part $\xi$, which tends to make $u$ spatially irregular.

$(\mathcal{H}$ is the so-called 'tight-binding Hamiltonian with diagonal disorder' considered in Anderson (1958).)

Depending on $\kappa$ and on the marginal law of $\xi$, the equation in $(0.1)$ can be used to model various physical and chemical phenomena. For instance, $t \rightarrow\left\{u(x, t): x \in \mathbb{Z}^{d}\right\}$ may describe the evolution of the density field of a chemical component in a catalytic reaction (Zel'dovich (1984)) or the average occupation field in a system of particles that branch and migrate (Dawson and Ivanoff (1978)). In these examples the role of $\xi$ is to act as a spatially inhomogeneous local rate of catalysis resp. branching. Other applications are: Fisher-Eigen equation in Darwinian evolution (Ebeling et al. (1984)); Burgers' equation with a random force in hydrodynamics (Carmona and Molchanov (1994)).

The following result gives a sufficient condition on $\xi$ to ensure that $(0.1)$ is actually applicable to such concrete situations. Let $\langle\cdot\rangle$ denote expectation w.r.t. the $\xi$-field. Let $Z=\{Z(t): t \geq 0\}$ denote simple random walk on $\mathbb{Z}^{d}$ jumping at rate $2 d \kappa$ (i.e., the Markov process with generator $\kappa \Delta)$. Write $P_{x}, E_{x}$ to denote probability and expectation on path space given $Z(0)=x$. 
Proposition 1 (Gärtner and Molchanov (1990)) If

$$
\left\langle\left[\frac{\xi_{+}(0)}{\log \xi_{+}(0)}\right]^{d}\right\rangle<\infty \quad \text { with } \xi_{+}(0)=\xi(0) \vee e,
$$

then (0.1) has a unique nonnegative solution $\xi$-a.s. This solution admits the Feynman-Kac representation

$$
u(x, t)=E_{x}\left(\exp \left[\int_{0}^{t} \xi(Z(s)) d s\right]\right) .
$$

Moreover, for all $t \geq 0$ the random field $\left\{u(x, t): x \in \mathbb{Z}^{d}\right\}$ is stationary and ergodic under translations.

The proof of Proposition 1, which is based on ideas from percolation, shows that in dimension $d \geq 2$ condition (0.5) is in fact necessary: if (0.5) fails then a.s. there is no nonnegative solution to $(0.1)$.

\subsection{Intermittency}

A discussion of some mathematical problems related to $(0.1)$ can be found in the recent memoir by Carmona and Molchanov (1994). In the present paper we shall be concerned with one particular aspect of (0.1), namely the occurrence of intermittency.

We shall henceforth assume that the cumulant generating function of the $\xi$-field is finite on the positive half axis:

$$
H(t)=\log \left\langle e^{t \xi(0)}\right\rangle<\infty \quad \text { for all } t \geq 0 .
$$

It is easily seen from the representation in (0.6) that assumption (0.7) is equivalent to all moments and correlations of the $u$-field being finite for all times (see also Lemmas 1 and 2 in Section 1).

Definition Let

$$
\Lambda_{k}(t)=\log \left\langle u^{k}(0, t)\right\rangle \quad(k=1,2, \ldots) .
$$

The system (0.1) is said to be intermittent if 1

$$
\lim _{t \rightarrow \infty}\left\{\frac{\Lambda_{l}(t)}{l}-\frac{\Lambda_{k}(t)}{k}\right\}=\infty \quad \text { for all } l>k \geq 1 .
$$

Qualitatively, (0.9) means that the $u$-field develops sparsely distributed high peaks as $t$ increases. These peaks give the dominant contribution to the moments as they become sparser and higher. Thus the landscape formed by $u$ is so irregular that the a.s. growth at a fixed site differs from the average growth in a large box.

\footnotetext{
${ }^{1}$ It is easily checked that (0.9) holds for all $l>k \geq 1$ iff it holds for $k=1, l=2$ (Gärtner and Molchanov (1990) Section 1.1)
} 
As is evident from $(0.1-0.2)$, peaks tend to grow in the vicinity of where the $\xi$-field is large (at a rate proportional to the field), but tend to be flattened out by the diffusion. By analogy with the theory of Anderson localization (see e.g. Fröhlich et al. (1985)), one may expect to find from a spectral analysis of the operator in (0.2) that the effect of the randomness in the $\xi$-field qualitatively dominates the effect of the diffusion term $\kappa \Delta$. This is indeed the case, as expressed by the following result.

Proposition 2 (Gärtner and Molchanov (1990)) If

$$
\xi(0) \neq \text { constant }
$$

then (0.1) is intermittent.

\subsection{Correlation structure: $(*)$ and Theorems 1-2}

Our goal in this paper is to show that there is a qualitative change in the structure of the intermittent peaks when the law of $\xi(0)$ is (in the vicinity of) the double exponential

$$
\operatorname{Prob}(\xi(0)>s)=\exp \left[-e^{s / \theta}\right] \quad(s \in \mathbb{R}) .
$$

Here $\theta \in(0, \infty)$ is a parameter that can be thought of as measuring the degree of disorder in the $\xi$-field, because the density associated with $(0.11)$ rapidly drops to zero outside the interval $[-\theta, \theta]$. Our main result, Theorem 1 below, gives the correlation coefficient of $u(x, t)$ and $u(y, t)$ for $x, y \in \mathbb{Z}^{d}$ fixed and $t \rightarrow \infty$. We shall see that what this result says is that the intermittent peaks have a particular asymptotic shape that depends on the ratio $\theta / \kappa$ (see Section 0.6).

To formulate Theorem 1 we introduce the following 1-dimensional nonlinear difference equation:

$$
\begin{aligned}
& \text { (*) } \Delta v+2 \rho v \log v=0 \text {, } \\
& v: \mathbb{Z} \rightarrow \mathbb{R}^{+}=(0, \infty), \rho=\theta / \kappa \text {. }
\end{aligned}
$$

We shall be interested in the ground states of $(*)$, i.e., the solutions in $l^{2}(\mathbb{Z})$ with minimal $l^{2}$-norm.

Theorem 1 Fix $\kappa, \theta \in(0, \infty)$ and put $\rho=\theta / \kappa$. Suppose that the law of $\xi(0)$ is given by (0.11). If there exists a $v_{\rho}: \mathbb{Z} \rightarrow \mathbb{R}^{+}$such that

A1. $v_{\rho}$ is a ground state of $(*)$,

A2. all other ground states are translations of $v_{p}$,

then for any $x, y \in \mathbb{Z}^{d}$

$$
\lim _{t \rightarrow \infty} \frac{\langle u(x, t) u(y, t)\rangle}{\left\langle u^{2}(0, t)\right\rangle}=\frac{1}{\left\|w_{\rho}\right\|_{\ell^{2}}^{2}} \sum_{z \in \mathbb{Z}^{d}} w_{\rho}(x+z) w_{\rho}(y+z),
$$

where $w_{\rho}: \mathbb{Z}^{d} \rightarrow \mathbb{R}^{+}$is given by

$$
w_{\rho}=\left(v_{\rho}\right)^{\otimes d} \text {. }
$$


Theorem 1, which will follow from Theorem 3 in Section 0.5, gives us a precise description of the correlation structure of the intermittent peaks provided assumptions A1-A2 are met. However, the verification of these assumptions is a nontrivial problem, due to the discrete nature of $(*)$. As a partial result we can offer the following theorem, which will be proved in Section 5.

Theorem 2 Let $\mathcal{V}_{\rho}=\left\{v_{\rho}: \mathbb{Z} \rightarrow \mathbb{R}^{+}: v_{\rho}\right.$ is a ground state of $\left.(*)\right\}$.

I. For all $\rho \in(0, \infty)$ :

(1) A1 holds, i.e., $\mathcal{V}_{\rho} \neq \emptyset$.

(2) $\mathcal{V}_{\rho}$ is compact in the $\ell^{2}$-metric modulo shifts. ${ }^{2}$

(3) For every centered $v_{\rho} \in \mathcal{V}_{\rho}:{ }^{3}$

(i) either $v_{\rho}(x)<v_{\rho}(0)$ for all $x \neq 0$ (single-point maximum) or $v_{\rho}(x)<$ $v_{\rho}(0)=v_{\rho}(1)$ for all $x \neq 0,1$ (double-point maximum);

(ii) $v_{p}$ is strictly unimodal, i.e., strictly monotone left and right of its maximum;

(iii) $v_{\rho}(x+1) / v_{\rho}(x) \sim 1 /(2 \rho x \log x)(x \rightarrow \infty)$, and similarly for $x \rightarrow-\infty$.

II. For $\rho$ sufficiently large:

(4) A2 holds, i.e., $\mathcal{V}_{p}$ is a singleton modulo shifts.

(5) The centered $v_{\rho}$ has a single-point maximum and is symmetric.

III. For any centered family $\left(v_{\rho}\right)_{\rho \in(0, \infty)}$ with $v_{\rho} \in \mathcal{V}_{\rho}$ :

(6) $\lim _{\rho \rightarrow \infty} v_{\rho}=\delta_{0}$ pointwise.

(7) $\lim _{\rho \rightarrow 0} v_{\rho}(\lfloor x / \sqrt{\rho}\rfloor)=\exp \left[\frac{1}{2}\left(1-x^{2}\right)\right]$ in $L^{2}(\mathbb{R})$ and uniformly on compacts in $\mathbb{R}$ (where $\lfloor\cdot\rfloor$ denotes the integer part).

Our estimates in Section 5 show that Theorem 2II holds when $\rho \geq 2 / \log \left(1+e^{-2}\right)$. Possibly it holds for all $\rho>0$, but we are unable to prove this. See Section 0.7 for a description of numerical work. ${ }^{4}$

Note that Theorem 2I(3)(iii) implies

$$
v_{\rho}(x)=\exp [-(1+o(1))|x| \log |x|] \quad(|x| \rightarrow \infty) .
$$

\footnotetext{
${ }^{2}$ For $v \in l^{2}(\mathbb{Z})$, let $[v]=\{v(\cdot+x): x \in \mathbb{Z}\}$ be the equivalence class given by the translations of $v$. For $\mathcal{V} \subseteq l^{2}(\mathbb{Z})$, let $[\mathcal{V}]=\{[v]: v \in \mathcal{V}\}$ be the set of equivalence classes of $\mathcal{V}$. We equip $\left[l^{2}(\mathbb{Z})\right]$ with the metric $\|[u]-[v]\|_{\ell^{2}}=\inf _{x \in \mathbb{Z}}\|u(\cdot)-v(\cdot+x)\|_{\ell^{2}}$. The statement in Theorem 2I(2) means that $\left[\mathcal{V}_{\rho}\right]$ is compact in the topology induced by this metric.

${ }^{3}$ We call $v \in l^{2}(\mathbb{Z})$ centered if $v(0)=\max _{x} v(x)$ and $v(x)<v(0)$ for $x<0$.

${ }^{4}$ The continuous version of (*) is trivial. In fact, $v^{\prime \prime}+2 \rho v \log v=0$ for $v: \mathbb{R} \rightarrow \mathbb{R}^{+}$has only one solution in $L^{2}(\mathbb{R})$ (modulo translations), namely $v_{\rho}(x)=\exp \left[\frac{1}{2}\left(1-\rho x^{2}\right)\right]$. Indeed, multiply by $v^{\prime}$ to see that any solution satisfies $\frac{1}{2}\left(v^{\prime}\right)^{2}+\rho v^{2}\left(\log v-\frac{1}{2}\right) \equiv A(A \in \mathbb{R})$. If $v \in L^{2}(\mathbb{R})$, then necessarily $A=0$ (compatible with $v(x), v^{\prime}(x) \rightarrow 0$ as $\left.|x| \rightarrow \infty\right)$. Substitute $v=\exp (f)$ to get $\frac{1}{2}\left(f^{\prime}\right)^{2}+\rho\left(f-\frac{1}{2}\right)=0$. The (twice continuously differentiable) solution is $f(x)=\frac{1}{2}-\frac{1}{2} \rho(x-B)^{2}(B \in \mathbb{R})$.
} 
So, in particular, $w_{\rho}$ defined in $(0.13)$ is an element of $\ell^{1}\left(\mathbb{Z}^{d}\right) \subseteq \ell^{2}\left(\mathbb{Z}^{d}\right)$.

\section{Remarks}

(A) The proof in Sections 2-4 will show that we do not require the law of $\xi(0)$ to be given precisely by $(0.11)$. What we actually need is that $H(t)$ defined in $(0.7)$ has the following asymptotic property:

$$
\lim _{t \rightarrow \infty} t H^{\prime \prime}(t)=\theta \quad \text { for some } \theta \in(0, \infty)
$$

The parameter $\theta$ in $(0.15)$ takes over the role of $\theta$ in $(0.11)$. For the double exponential in $(0.11)$ we have $H(t)=\log \Gamma(\theta t+1)$, which indeed satisfies $(0.15)$.

(B) The proof in Sections $2-4$ will also show that if $\lim _{t \rightarrow \infty} t H^{\prime \prime}(t)=0$ or $\infty$, then the 1.h.s. of $(0.12)$ is the constant function 1 resp. $\delta_{x, y}$ (compatible with Theorem 2III). Thus, the distributions characterized by (0.15) form the critical class with an interesting correlation structure.

\subsection{A variational problem: (**) and Proposition 3}

In view of (0.6), it is no surprise that the proof of Theorem 1 uses large deviation theory and that the nonlinear equation $(*)$ comes from an associated variational problem. We shall formulate this variational problem here. In Section 0.5 it will reappear in Theorem 3 , which describes the asymptotic behavior of the 1-st and 2-nd moments of the field $\left\{u(x, t): x \in \mathbb{Z}^{d}\right\}$ and which is a refinement of Theorem 1 .

Let $\mathcal{P}_{d}=\mathcal{P}\left(\mathbb{Z}^{d}\right)$ denote the set of probability measures on $\mathbb{Z}^{d}$. On $\mathcal{P}_{d}$ define the functionals

$$
\begin{aligned}
& I_{d}(p)=\sum_{\{x, y\}:|x-y|=1}(\sqrt{p(x)}-\sqrt{p(y)})^{2} \\
& J_{d}(p)=-\sum_{x} p(x) \log p(x) .
\end{aligned}
$$

Define

$(* *) \quad \chi(\rho)=\frac{1}{2 d} \inf _{p \in \mathcal{P}_{d}}\left\{I_{d}(p)+\rho J_{d}(p)\right\}$.

We have $0 \leq \chi(\rho) \leq 1$ (because $I_{d}, J_{d} \geq 0$ resp. $I_{d}\left(\delta_{0}\right)=2 d, J_{d}\left(\delta_{0}\right)=0$ ). Moreover, $\rho \rightarrow \chi(\rho)$ is nondecreasing and concave with limits $\lim _{\rho \rightarrow 0} \chi(\rho)=0$ resp. $\lim _{\rho \rightarrow \infty} \chi(\rho)=1$.

The following proposition will be proved in Section 5.1 and provides the link between $(*)$ and $(* *)$.

Proposition 3 For all $\rho \in(0, \infty)$ :

(1) (**) has a minimum.

(2) $p$ is a minimizer of $(* *)$ iff $p=\otimes_{i=1}^{d}\left(v_{i}^{2} /\left\|v_{i}\right\|_{\ell^{2}}^{2}\right)$ with $v_{i}$ any ground state of $(*)$.

(3) $\chi(\rho)=\rho \log \|v\|_{\ell^{2}}$ with $v$ any ground state of $(*)$. 
Note that $\chi(\rho)$ does not depend on the dimension $d$. Theorem 2III(7) and Proposition $3(3)$ imply that $\chi(\rho)=\frac{\rho}{4}\left[\log (1 / \rho)+\log \left(\pi e^{2}\right)+o(1)\right](\rho \rightarrow 0)$. Thus $\chi$ has infinite slope at $\rho=0$.

\subsection{Asymptotics of the 1-st and 2-nd moments: Theorem 3}

The $\chi$-function appears in the following asymptotic expansions. Recall the definition of $H$ in $(0.7)$ and of $w_{p}$ in $(0.13)$.

Theorem 3 Fix $\kappa, \theta \in(0, \infty)$ and put $\rho=\theta / \kappa$. Suppose that the law of $\xi(0)$ satisfies (0.15) and suppose that $A 1-A 2$ in Theorem 1 hold. Then for $x, y \in \mathbb{Z}^{d}$ fixed and $t \rightarrow \infty$

$$
\begin{aligned}
\langle u(x, t)\rangle= & \left\{\sum_{z \in \mathbb{Z}^{d}} w_{\rho}(x+z)\right\} \\
& \times \exp \left[H(t)-\chi(\rho) 2 d \kappa t+C_{1}(\rho, \kappa t)+o(1)\right] \\
\langle u(x, t) u(y, t)\rangle= & \left\{\sum_{z \in \mathbb{Z}^{d}} w_{\rho}(x+z) w_{\rho}(y+z)\right\} \\
& \times \exp \left[H(2 t)-\chi(\rho) 4 d \kappa t+C_{2}(\rho, \kappa t)+o(1)\right],
\end{aligned}
$$

where $C_{1}(\rho, \kappa t), C_{2}(\rho, \kappa t)$ are functions of order o(t) that are independent (!) of $x, y$.

Theorem 3, which will be proved in Sections 2-4, obviously implies Theorem 1. It is crucial that the expansions in $(0.18-0.19)$ are independent of $x, y$ up to the error term $o(1)$. The dependence on $x, y$ sits solely in the prefactors. We shall see in Section 2 that the functions $C_{1}, C_{2}$ are in fact very sensitive to the precise form of the function $H$, but that the prefactors only depend on the asymptotic behavior of $H$ assumed in (0.15). It is beyond the scope of the present paper to identify $C_{1}, C_{2}$.

\subsection{Discussion}

The double exponential is nondegenerate and so, according to Proposition 2, the $u$-field is intermittent. This means that the $k$-th moment is controlled by a different class of peaks for each $k$. Moreover, as $k$ increases the peaks in the ' $k$-class' become sparser but higher (recall $(0.8-0.9))$.

For $t$ large but fixed, the ergodic theorem tells us that the ratio of 2 -nd moments appearing in the 1.h.s. of (0.12) essentially counts how often two peaks in the class $k=2$ are seen at a relative distance $y-x$ resp. 0 in a large box. In other words, if we think of the peaks as located on random islands, then the ratio essentially counts the pairs of sites in a large box that are at distance $y-x$ resp. 0 and both belong to an island. It is in this sense that the correlation structure established in Theorem 1 is related to the typical size of the islands. 
Peaks grow in the vicinity of where the $\xi$-field is large, but are not fully localized on the local maxima of $\xi$ because the diffusion term $\kappa \Delta$ has a tendency to spread them out. Now, the double exponential defined in (0.11) makes a sharp drop beyond the value $\theta$. Therefore, the larger $\theta$ the larger the local maxima of $\xi$ and hence the more localized the peaks. On the other hand, the larger $\kappa$ the faster the diffusion and hence the less localized the peaks. Theorem 1 shows that, apparently, it is the parameter $\rho=\theta / \kappa$ that controls the size of the islands. More specifically, if $c_{\rho}(x, y)$ denotes the r.h.s. of $(0.12)$, then we see from Theorem 2III that

$$
\begin{array}{lll}
\lim _{\rho \rightarrow \infty} c_{\rho}(x, y) & =\delta_{x, y} & \left(x, y \in \mathbb{Z}^{d}\right) \\
\lim _{\rho \rightarrow 0} c_{\rho}(\lfloor x / \sqrt{\rho}\rfloor,\lfloor y / \sqrt{\rho}\rfloor) & =e^{-\frac{1}{4}|x-y|^{2}} & \left(x, y \in \mathbb{R}^{d}\right) .
\end{array}
$$

The second statement says that an island in the class $k=2$ has widths in the $d$ lattice directions that are of order $1 / \sqrt{\rho}$ for small $\rho$. In other words, the long-time correlation length of the $u$-field is of order $1 / \sqrt{\rho}$ for small $\rho$.

The result in Theorem 3 should be interpreted as follows. Let the highest peaks in the islands corresponding to the classes $k=1,2$ have heights $h_{1}(t), h_{2}(t)$ and densities $d_{1}(t), d_{2}(t)$. If $x_{1}(t), x_{2}(t)$ denote the centers of some randomly chosen peaks, then $(0.18-$ $0.19)$ tell us that

$$
\begin{aligned}
& k=1: \quad u\left(x_{1}(t)+x, t\right)=\frac{w_{\rho}(x)}{w_{\rho}(0)} h_{1}(t) \\
& k=2: \quad u\left(x_{2}(t)+x, t\right)=\frac{w_{\rho}(x)}{w_{\rho}(0)} h_{2}(t)
\end{aligned}
$$

and

$$
\begin{aligned}
& d_{1}(t) h_{1}(t)=w_{\rho}(0) \exp \left[H(t)-\chi(\rho) 2 d \kappa t+C_{1}(\rho, \kappa t)+o(1)\right] \\
& d_{2}(t) h_{2}^{2}(t)=w_{\rho}^{2}(0) \exp \left[H(2 t)-\chi(\rho) 4 d \kappa t+C_{2}(\rho, \kappa t)+o(1)\right] .
\end{aligned}
$$

In other words, modulo an unknown height and an unknown density, the peaks have a non-random shape that is given by $w_{\rho}$ for both classes. (The same result holds for the classes $k \geq 3$, but these will not be considered in the present paper.)

Thus, the results in Theorems 1-3 give us a picture of the correlation structure of the $u$-field that is much more detailed than the notion of intermittency. Indeed, while intermittency tells us that the peaks occur on sparse islands, our result tells us that the peaks

(1) contract to single points when $\rho=\infty$;

(2) grow unboundedly when $\rho=0$;

(3) develop an interesting finite structure when $\rho \in(0, \infty)$. 


\section{$0.7 \quad$ Numerical study of $(*)$}

For each $\rho \in(0, \infty)$ there are two centered symmetric solutions of $(*)$, one with a singlepoint maximum and one with a double-point maximum. Let $v^{(1)}$ and $v^{(2)}$ denote these solutions, respectively. Then

$$
\begin{array}{ll}
v^{(1)}(0)>v^{(1)}(1)>v^{(1)}(2)>\ldots & v^{(1)}(-x)=v^{(1)}(x) \quad(x \in \mathbb{Z}) \\
v^{(2)}(0)=v^{(2)}(1)>v^{(2)}(2)>\ldots & v^{(2)}(-x)=v^{(2)}(x+1) \quad(x \in \mathbb{Z}) .
\end{array}
$$

Now, we may ask which of these two solutions has the smaller $l^{2}$-norm and whether there exist values of the parameter $\rho$ for which the norms coincide. We have done high precision computations with the package Mathematica. These strongly indicate that always $\left\|v^{(2)}\right\|_{\ell^{2}}>\left\|v^{(1)}\right\|_{\ell^{2}}$, although for small values of $\rho$ the difference $\delta^{2}=\left\|v^{(2)}\right\|_{\ell^{2}}^{2}-\left\|v^{(1)}\right\|_{\ell^{2}}^{2}$ is extremely small:

\begin{tabular}{c|cccccc}
$\rho$ & 2 & 1 & 0.5 & 0.25 & 0.1 & 0.05 \\
\hline$\left\|v^{(1)}\right\|_{\ell^{2}}^{2}$ & 2.49 & 4.38 & 6.58 & 9.48 & 15.1 & 21.5 \\
$\delta^{2}$ & $6.8110^{-1}$ & $9.5810^{-2}$ & $1.2310^{-4}$ & $6.7510^{-11}$ & $2.4710^{-30}$ & $3.6910^{-63}$
\end{tabular}

If there would be no other candidates for the centered solution of $(*)$ with minimal $l^{2}$ norm (which we do not know!), then these numerics would lead us to the conclusion that for all $\rho \in(0, \infty)$ the minimal $l^{2}$-solution of $(*)$ is uniquely given by $v^{(1)}$ modulo shifts (i.e., Theorem 2II would hold for all $\rho \in(0, \infty))$. Therefore, theoretically, the high peaks of the $u$-field contributing to the moments have a unique shape determined by $v^{(1)}$, as explained in Section 0.6. However, practically, for small $\rho$ also the peaks with shape $v^{(2)}$ have to be taken into account, unless the time is extremely large.

Let us briefly explain our numerical algorithm, which is based on the following observation. The symmetric solutions of $(*)$ corresponding to an initial datum $v(0)$ are: (i) not strictly decreasing when $v(0)$ is small, (ii) not everywhere strictly positive when $v(0)$ is large. The algorithm varies $v(0)$ until both of these failures are removed (as is required by Theorem $2 \mathrm{I}(3)(\mathrm{i}-\mathrm{ii})$ ). Given an initial datum $v(0)$, we compute $v(1), \ldots, v(N)$ (with $N$ ranging from 25 to 75 depending on $\rho$ ) by the following rules:

$$
\begin{aligned}
v(1) & :=v(0)[1-\rho \log v(0)] \quad & \text { for the single-point maximum, } \\
v(1) & :=v(0) & \text { for the double-point maximum, } \\
v(n+1) & :=v(n)[2-2 \rho \log v(n)]-v(n-1), & \text { if } v(n)>0, \\
v(n+1) & :=v(n), & \text { if } v(n) \leq 0,
\end{aligned}
$$

for $n=1, \ldots, N-1$. The correct initial datum $v(0)$ is then computed by using the following interval approximation. We start with the interval $\left[a_{0}, b_{0}\right]:=[1,2]$ and take $v(0):=\left(a_{0}+b_{0}\right) / 2$. Then we compute $v(1), \ldots, v(N)$ in accordance with the above rules. If this sequence of numbers is not strictly decreasing or if $v(N)>0$, then we put $a_{1}:=$ $\left(a_{0}+b_{0}\right) / 2$ and $b_{1}:=b_{0}$. Otherwise we put $a_{1}:=a_{0}$ and $b_{1}:=\left(a_{0}+b_{0}\right) / 2$. We then take $v(0):=\left(a_{1}+b_{1}\right) / 2$, etc. This process is iterated $m$ times until $b_{m}-a_{m}$ becomes less than $10^{-100}$. 


\subsection{Related work}

As a further reference to intermittency we mention the following papers. Antal (1995) studies the survival of simple random walk on $\mathbb{Z}^{d}$ in a random field of traps with density $c \in(0,1)$. This model is equivalent to $(0.1)$ when $\xi(0)$ takes the values $-\infty$ and 0 with

probability $c$ resp. $1-c$ (as can be seen from $(0.6)$ ). His analysis shows that at time $t$ the 'islands' have a size of order $t^{1 /(d+2)}$. Greven and den Hollander (1992) and Sznitman (1994) study models related to (0.1) when a drift is added to the diffusive part $\kappa \Delta$ and the $\xi$-field is bounded. It turns out that in this situation there is a critical value for the drift, below which the a.s. exponential growth rate and the box-averaged exponential growth rate are the same but above which they are not. This fact indicates that for a bounded $\xi$-field the occurrence of intermittency depends on the strength of the drift.

Finally, Bolthausen and Schmock (preprint 1994) study simple random walk on $\mathbb{Z}^{d}$ with a self-attractive interaction inversely proportional to time, which technically leads to similar questions. They show that this process is localized and has a limit law that can be identified in terms of a variational problem and an associated nonlinear difference equation similar in nature to our $(* *)$ and $(*)$. We have picked up several ideas from their paper, although the functionals arising in our context require a modified approach.

The outline of the rest of this paper is as follows. In Section 1 we give a heuristic explanation of Theorem 3. In Section 2 we formulate the main steps in the proof of Theorem 3 by listing six key propositions. These propositions are proved in Sections $3-4$. In Section 5 we prove Theorem 2 and Proposition 3. Theorem 1 is implied by Theorem 3, as was pointed out above.

\section{Heuristic explanation of Theorem 3}

In this section we explain where (0.18-0.19) come from. We give a heuristic argument showing how the quantity $\chi(\rho)$ arises from large deviations of local times associated with our simple random walk $Z=\{Z(t): t \geq 0\}$, and how the higher order terms in the expansions require an analysis of the corrections to large deviations.

\subsection{Expansion for the 1-st moment}

Return to the Feynman-Kac representation (0.6). Define the local times

$$
\ell_{t}(z)=\int_{0}^{t} 1_{\{Z(s)=z\}} d s \quad\left(z \in \mathbb{Z}^{d}, t \geq 0\right)
$$

Lemma 1 For all $x \in \mathbb{Z}^{d}$ and $t \geq 0$

$$
\langle u(x, t)\rangle=E_{x}\left(\exp \left[\sum_{z \in \mathbb{Z}^{d}} H\left(\ell_{t}(z)\right)\right]\right) .
$$


Proof. Use (1.1) to rewrite $(0.6)$ as $u(x, t)=E_{x}\left(\exp \left[\sum_{z} \xi(z) \ell_{t}(z)\right]\right)$. Take the expectation over $\xi$, use Fubini's theorem, and use $(0.7)$ in combination with the i.i.d. property of $\xi$. $\square$

Since $\sum_{z} \ell_{t}(z)=t$, the exponent in (1.2) may be rewritten as

$$
\sum_{z} H\left(\ell_{t}(z)\right)=H(t)+t \sum_{z} \frac{1}{t}\left[H\left(\frac{\ell_{t}(z)}{t} t\right)-\frac{\ell_{t}(z)}{t} H(t)\right] .
$$

Now, $H$ has the following scaling property (which is implied by $(0.15)$ ):

$$
\lim _{t \rightarrow \infty} \frac{1}{t}[H(c t)-c H(t)]=\theta c \log c \quad \text { uniformly in } c \in[0,1] .
$$

It therefore seems plausible from (1.3) that as $t \rightarrow \infty$

$$
\sum_{z} H\left(\ell_{t}(z)\right)=H(t)+t \theta \sum_{z} \frac{\ell_{t}(z)}{t} \log \left(\frac{\ell_{t}(z)}{t}\right)+o(t)
$$

Let $L_{t}$ denote the occupation time measure associated with $Z$, i.e.,

$$
L_{t}(\cdot)=\frac{\ell_{t}(\cdot)}{t}
$$

Then, recalling the definition of the functional $J_{d}$ in $(0.17)$, we see that the sum in the r.h.s. of (1.5) equals $-J_{d}\left(L_{t}\right)$. Substituting (1.5) into (1.2) we thus get

$$
\langle u(x, t)\rangle=E_{x}\left(\exp \left[H(t)-t \theta J_{d}\left(L_{t}\right)+o(t)\right]\right) .
$$

Next, according to the Donsker-Varadhan large deviation theory, $L_{t}$ satisfies the weak large deviation principle on $\mathcal{P}_{d}$ with rate function $\kappa I_{d}$, where $I_{d}$ is the functional in $(0.16)$ (Deuschel and Stroock (1989), Theorem 3.2.17). Thus it seems plausible from (1.7) that as $t \rightarrow \infty$

$$
\langle u(x, t)\rangle=\exp \left[H(t)-t \inf _{p \in \mathcal{P}_{d}}\left\{\kappa I_{d}(p)+\theta J_{d}(p)\right\}+o(t)\right] .
$$

The infimum in the exponent is precisely $\chi(\theta / \kappa) 2 d \kappa$, with $\chi$ defined in $(* *)$. So this explains the first two terms of the expansion in (0.18).

A rigorous proof of (1.8) is given in Gärtner and Molchanov (preprint 1996). The proof uses a standard compactification method:

(i) Pick a large box $T_{N}=(-N, N]^{d} \cap \mathbb{Z}^{d}$.

(ii) Get an upper bound on $\langle u(x, t)\rangle$ by wrapping the random walk around $T_{N}$, i.e., define $\ell_{t}^{N}(z)=\sum_{z^{\prime} \in 2 N \mathbb{Z}^{d}} \ell_{t}\left(z+z^{\prime}\right)\left(z \in T_{N}\right)$ and use that $\sum_{z \in \mathbb{Z}^{d}} H\left(\ell_{t}(z)\right) \leq \sum_{z \in T_{N}} H\left(\ell_{t}^{N}(z)\right)$ (because $H(0)=0$ and $t \rightarrow H(t)$ is convex).

(iii) Get a lower bound on $\langle u(x, t)\rangle$ by killing the random walk at the boundary of $T_{N}$, i.e., add the indicator of the event that $\ell_{t}(z)=0$ for all $z \in\left(T_{N}^{c} \cup \partial T_{N}\right)$. 
(iv) Use the full large deviation principle for $L_{t}^{N}(\cdot)=\ell_{t}^{N}(\cdot) / t$ on $T_{N}$. This leads to an expansion as in (1.8), but with an $N$-dependent upper resp. lower variational problem. In these variational problems the same functionals as in $(0.16-0.17)$ appear, but now defined for $p \in \mathcal{P}_{d}\left(T_{N}\right)$ with periodic resp. Dirichlet boundary condition.

(v) Let $N \rightarrow \infty$ and show that both variational problems converge to (**).

To get the full expansion in (0.18) we need to go one step further and show that the term $\exp [o(t)]$ in (1.8) is actually $\left\{\sum_{z} w_{\rho}(x+z)\right\} \exp \left[C_{1}(\rho, \kappa t)+o(1)\right]$. To achieve this we must analyze the corrections to the large deviation behavior of $L_{t}$. This will be done in Sections 2-4 and amounts to studying the local times of a transformed random walk, chosen in such a way that its occupation time measure performs random fluctuations around the minimizer $w_{\rho}^{2} /\left\|w_{\rho}\right\|_{\ell^{2}}^{2}$ of our variational problem (**) (modulo shifts). More precisely, we consider the random walk

$$
Z_{\rho}=\left\{Z_{\rho}(s): s \geq 0\right\}
$$

whose generator $G_{\rho}$ is

$$
\left(G_{\rho} f\right)(x)=\kappa \sum_{y:|y-x|=1} \frac{w_{\rho}(y)}{w_{\rho}(x)}[f(y)-f(x)]
$$

considered as a self-adjoint operator on $\ell^{2}\left(\mathbb{Z}^{d} ; w_{\rho}^{2} /\left\|w_{\rho}\right\|_{\ell^{2}}^{2}\right)$. The crucial point is that the invariant probability measure of $Z_{\rho}$ is precisely $w_{\rho}^{2} /\left\|w_{\rho}\right\|_{\ell^{2}}^{2}$. The absolute continuous transformation from $Z$ to $Z_{\rho}$ gives rise to the prefactor in (0.18) and to the first two terms in the expansion. The higher order terms in the expansion are therefore determined by the fluctuations of $L_{t}$ under the law of $Z_{\rho}$. The details are worked out in Sections 2-4.

Note that $Z_{\rho}$ has a drift towards 0 that increases rapidly with the distance to 0 (see (0.13) and Theorem 2I(3)(iii)). Thus it has strong ergodic properties.

\subsection{Expansion for the 2-nd moment}

The heuristic explanation of $(0.19)$ is in the same spirit. This time the starting point is the following analogue of Lemma 1.

Lemma 2 For all $x, y \in \mathbb{Z}^{d}$ and $t \geq 0$

$$
\langle u(x, t) u(y, t)\rangle=E_{x, y}\left(\exp \left[\sum_{z \in \mathbb{Z}^{d}} H\left(\hat{\ell}_{t}(z)\right)\right]\right),
$$

where $E_{x, y}=E_{x} \otimes E_{y}$ and

$$
\hat{\ell}_{t}(\cdot)=\ell_{t}^{1}(\cdot)+\ell_{t}^{2}(\cdot)
$$

is the sum of the local times of two independent copies of $Z$ starting at $x$ resp. $y$. 
Proof. Same as for Lemma 1. Use (0.6).

An argument similar to (1.3-1.8) produces the first two terms of the expansion in (0.19). Namely, the analogue of (1.8) reads

$$
\begin{aligned}
& \langle u(x, t) u(y, t)\rangle= \\
& \exp \left[H(2 t)-2 t \inf _{p^{1}, p^{2} \in \mathcal{P}_{d}}\left\{\kappa \frac{1}{2}\left(I_{d}\left(p^{1}\right)+I_{d}\left(p^{2}\right)\right)+\theta J_{d}\left(\frac{1}{2}\left(p^{1}+p^{2}\right)\right)\right\}+o(t)\right] .
\end{aligned}
$$

Because $p \rightarrow J_{d}(p)$ is strictly concave, the infimum reduces to $p^{1}=p^{2}=p$ with $p \in \mathcal{P}_{d}$, which again equals $\chi(\kappa / \theta) 2 d \kappa$ (see Gärtner and Molchanov (preprint 1996) for a rigorous proof). To get the full expansion will amount to studying the occupation time measure

$$
\hat{L}_{t}(\cdot)=\frac{1}{2 t} \hat{\ell}_{t}(\cdot)
$$

associated with two independent copies of the transformed random walk $Z_{\rho}$ defined in (1.91.10). The details are worked out in Sections 2-4. Again, the prefactor and the first two terms in (0.19) arise through the absolute continuous transformation from $Z$ to $Z_{\rho}$, the higher order terms through the fluctuations of $\hat{L}_{t}$ under the law of the two copies of $Z_{\rho}$.

\section{Main propositions}

In this section we outline the main steps in the proof of $(0.19)$ in Theorem 3 . These steps are formulated as Propositions 4-9 in Sections 2.1-2.6 below. The proof of these propositions will be given in Sections $3-4$, the proof of (0.19) subject to these propositions in Section 2.7. It will become clear from the whole construction that $(0.18)$ in Theorem 3 holds too, namely, via a straightforward simplification of the arguments given below to one instead of two random walks (compare Lemmas 1 and 2).

Our starting point is Lemma 2, which gives us a representation for $\langle u(x, t) u(y, t)\rangle$ in terms of $H$, the cumulant generating function of the $\xi$-field, and $\hat{\ell}_{t}=\ell_{t}^{1}+\ell_{t}^{2}$, the sum of the local time functions of two independent simple random walks with step rate $2 d \kappa$. Throughout the sequel it will be assumed that $H$ satisfies the condition in (0.15). For ease of notation we shall abbreviate

$$
\sum_{z \in \mathbb{Z}^{d}} H\left(\hat{\ell}_{t}(z)\right)=H \circ \hat{\ell}_{t}
$$

Throughout Sections 2-4 assumptions A1-A2 in Theorem 1 are in force.

\subsection{Clumping of the local times: Proposition 4}

Proposition 4 below states that the asymptotic behavior of the 2 -nd moments is controlled by paths whose occupation time measure $\hat{L}_{t}=\hat{\ell}_{t} / 2 t$ is close to a minimizer of (**). This property will allow us in Section 2.2 to truncate $\mathbb{Z}^{d}$. 
Let $\mathcal{M}$ denote the class of minimizers of (**). By assumptions A1-A2 in Theorem 1 in combination with Proposition $3(2), \mathcal{M}$ is a singleton modulo shifts.

For $\epsilon>0$, define

$$
\mathcal{U}_{\epsilon}=\left\{\mu \in \mathcal{P}\left(\mathbb{Z}^{d}\right):\|\mu-\nu\|_{\ell^{1}}<\epsilon \text { for some } \nu \in \mathcal{M}\right\}
$$

Proposition 4 Fix $x, y \in \mathbb{Z}^{d}$. For every $\epsilon>0$ there exists a $\delta>0$ such that

$$
E_{x, y}\left(\exp \left[H \circ \hat{\ell}_{t}\right] 1\left\{\frac{1}{2 t} \hat{\ell}_{t} \in \mathcal{U}_{\epsilon}\right\}\right) \geq\left(1-e^{-\delta t}\right) E_{x, y}\left(\exp \left[H \circ \hat{\ell}_{t}\right]\right)
$$

for all $t \geq 0$.

The proof of Proposition 4 is in Section 3.1 and is difficult for the following reason. From the full large deviation principle on the box $T_{N}=(-N, N]^{d} \cap \mathbb{Z}^{d}$ we know that for large $t$ the periodized occupation time measure, defined by $\hat{L}_{t}^{N}(z)=\sum_{z^{\prime} \in 2 N \mathbb{Z}^{d}} \hat{L}_{t}\left(z+z^{\prime}\right)\left(z \in T_{N}\right)$, is close to a minimizer of the periodized variational problem (see Section 1.1). However, this does not imply that $\hat{L}_{t}$ is close to a minimizer of $(* *)$. Essentially, what we must show is that the main contribution comes from paths whose local times are concentrated in one large box and not in two or more boxes separated by some distance. Namely, this precisely guarantees that $\hat{L}_{t}$ is close to $\hat{L}_{t}^{N}$ modulo a shift. We can then use the full large deviation principle on $T_{N}$, and Proposition 4 will follow by showing that the minimizers of the periodized variational problem are close to the minimizers of $(* *)$ when $N$ is large.

\subsection{Centering and truncation of the local times: Proposition 5}

For $\epsilon>0$ and $z \in \mathbb{Z}^{d}$, define (see footnote 3 )

$$
\mathcal{U}_{\epsilon}(z)=\left\{\mu \in \mathcal{P}\left(\mathbb{Z}^{d}\right):\|\mu-\nu\|_{\ell^{1}}<\epsilon \text { for some } \nu \in \mathcal{M} \text { centered at } z\right\} .
$$

By Theorems $2 \mathrm{I}(2)$ and $2 \mathrm{I}(3)(\mathrm{i})$, the $\mathcal{U}_{\epsilon}(z)$ 's for different $z$ 's are disjoint when $\epsilon$ is small enough. Write out

$$
\begin{aligned}
& E_{x, y}\left(\exp \left[H \circ \hat{\ell}_{t}\right] 1\left\{\frac{1}{2 t} \hat{\ell}_{t} \in \mathcal{U}_{\epsilon}\right\}\right) \\
& \quad=\sum_{z \in \mathbb{Z}^{d}} E_{x, y}\left(\exp \left[H \circ \hat{\ell}_{t}\right] 1\left\{\frac{1}{2 t} \hat{\ell}_{t} \in \mathcal{U}_{\epsilon}(z)\right\}\right) \\
& \quad=\sum_{z \in \mathbb{Z}^{d}} E_{x-z, y-z}\left(\exp \left[H \circ \hat{\ell}_{t}\right] 1\left\{\frac{1}{2 t} \hat{\ell}_{t} \in \mathcal{U}_{\epsilon}(0)\right\}\right) .
\end{aligned}
$$

Proposition 5 below is an estimate on the $x, y$-dependence of the summand in the r.h.s. of (2.5). This estimate implies that the summation over $z$ and the limit $t \rightarrow \infty$ may be interchanged. This will allow us in Sections 2.3-2.7 to first compute the asymptotics of the summand for fixed $x^{\prime}=x-z, y^{\prime}=y-z$ and $t \rightarrow \infty$ and afterwards carry out the summation over $z$. 
Proposition 5 There exist $A, \alpha>0$ and $t_{0}, \epsilon_{0}, R_{0}>0$ such that

$$
E_{x, y}\left(\exp \left[H \circ \hat{\ell}_{t}\right] 1\left\{\frac{1}{2 t} \hat{\ell}_{t} \in \mathcal{U}_{\epsilon}(0)\right\}\right) \leq A e^{-\alpha(|x|+|y|)} E_{0,0}\left(\exp \left[H \circ \hat{\ell}_{t}\right]\right)
$$

for all $t \geq t_{0}$, all $0<\epsilon \leq \epsilon_{0}$ and all $x, y \notin T_{R_{0}}$ (with $|x|$ the lattice norm of $x$ ).

The idea behind this estimate is that when the two random walks are forced to build up their local times in the neighborhood of the origin, then this will be harder to do when they start far away from the origin then when they start at the origin.

The prefactor in the r.h.s. of (2.6) is summable over $x, y \notin T_{R_{0}}$, showing that the remote terms in the r.h.s. of (2.5) are negligible uniformly in $t$.

Let $v_{\rho}: \mathbb{Z} \rightarrow \mathbb{R}^{+}$be the unique centered ground state of $(*)$. Let $w_{\rho}: \mathbb{Z}^{d} \rightarrow \mathbb{R}^{+}$be the product function $w_{\rho}=\left(v_{\rho}\right)^{\otimes d}$ in $(0.13)$ and define $p_{\rho}=w_{\rho}^{2} /\left\|w_{\rho}\right\|_{\ell^{2}}^{2}$. Then, by assumptions A1-A2 in Theorem 1 in combination with Proposition 3(2), $p_{\rho} \in \mathcal{P}_{d}$ is the unique centered minimizer of $(* *)$. Henceforth, instead of $\mathcal{U}_{\epsilon}(0)$ we shall write $\mathcal{U}_{\epsilon}\left(p_{\rho}\right)$, the $\epsilon$-neighborhood of $p_{\rho}$. In Sections 2.5-2.6 we shall be able to use Propositions 4 and 5 to expand $H \circ \hat{\ell}_{t}$ around $H \circ\left(2 t p_{\rho}\right)$. But before that we need some preparations.

\subsection{Two time scales: Proposition 6}

In order to do the expansion we shall need an estimate in the spirit of Proposition 5 but with two times $0 \leq t \leq T$. For $R>0$ define

$$
\hat{\sigma}_{R}=\inf \left\{s \geq 0: Z^{1}(s) \notin T_{R} \text { or } Z^{2}(s) \notin T_{R}\right\} .
$$

Proposition 6 Fix $x, y \in \mathbb{Z}^{d}$. There exist $A, \alpha>0$ and $T_{0}, \delta_{0}, \epsilon_{0}, R_{0}>0$ such that

$$
\begin{gathered}
E_{x, y}\left(\exp \left[H \circ \hat{\ell}_{T}\right] 1\left\{\frac{1}{2 T} \hat{\ell}_{T} \in \mathcal{U}_{\epsilon}\left(p_{\rho}\right)\right\} 1\left\{\hat{\sigma}_{R} \leq t\right\}\right) \\
\leq A t R^{d-1} e^{-\alpha R} E_{x, y}\left(\exp \left[H \circ \hat{\ell}_{T}\right]\right)
\end{gathered}
$$

for all $T \geq T_{0}$, all $t \geq 0$ with $t / T \leq \delta_{0}$, all $0<\epsilon \leq \epsilon_{0}$ and all $R \geq R_{0}$.

Note that $T$ takes over the role that $t$ was playing in the previous propositions, and that $t$ is now used as an auxiliary time. We shall henceforth stick to this notation.

Proposition 6 states that the main contribution comes from paths that do not move out of a large box before time $t$ uniformly in the length $T$ of the path.

Incidentally, the restrictions on $t, \epsilon, x, y$ in Proposition 5 resp. $T, t, \epsilon, R$ in Proposition 6 are partly an artefact of our proofs in Sections 3.2-3.3. However, these restrictions will not bother us in what follows. 


\subsection{Transformation of the random walk: Proposition 7}

In order to exploit Propositions 4-6 we shall make an absolute continuous transformation from our reference random walk with generator $\kappa \Delta$ to a new random walk whose generator $G_{\rho}$ is chosen as in (1.10). The point is that $G_{\rho}$ has precisely $p_{\rho}=w_{\rho}^{2} /\left\|w_{\rho}\right\|_{\ell^{2}}^{2}$ as its unique invariant probability measure (see Section 4.1). Thus, under the law of the random walk driven by $G_{\rho}$ and for large $T$, we have that $L_{T}^{i}=\ell_{T}^{i} / T(i=1,2)$ are close to $p_{\rho}$ with probability close to 1 , and hence so is $\hat{L}_{T}=\hat{\ell}_{T} / 2 T=\left(L_{T}^{1}+L_{T}^{2}\right) / 2$. Write $P_{x, y}^{\rho}=P_{x}^{\rho} \otimes P_{y}^{\rho}$ and $E_{x, y}^{\rho}=E_{x}^{\rho} \otimes E_{y}^{\rho}$ to denote the joint probability and expectation for two independent random walks driven by $G_{\rho}$ and starting at $x$ resp. $y$.

Proposition 7 For all $0 \leq t \leq T$, all $\epsilon, R>0$ and all $x, y \in \mathbb{Z}^{d}$

$$
\begin{aligned}
& E_{x, y}\left(\exp \left[H \circ \hat{\ell}_{T}\right] 1\left\{\frac{1}{2 T} \hat{\ell}_{T} \in \mathcal{U}_{\epsilon}\left(p_{\rho}\right)\right\} 1\left\{\hat{\sigma}_{R}>t\right\}\right) \\
& =\sqrt{p_{\rho}(x) p_{\rho}(y)} \exp [H(2 T)-\chi(\rho) 4 d \kappa T] \\
& \quad \times E_{x, y}^{\rho}\left(\exp \left[F_{T}\left(\hat{L}_{T}\right)\right] \frac{1}{\sqrt{p_{\rho}\left(Z^{1}(T)\right) p_{\rho}\left(Z^{2}(T)\right)}} 1\left\{\hat{L}_{T} \in \mathcal{U}_{\epsilon}\left(p_{\rho}\right)\right\} 1\left\{\hat{\sigma}_{R}>t\right\}\right),
\end{aligned}
$$

where $\hat{\sigma}_{R}$ is defined in (2.7) and

$$
F_{T}\left(\hat{L}_{T}\right)=\sum_{z}\left\{H\left(2 T \hat{L}_{T}(z)\right)-\hat{L}_{T}(z) H(2 T)-2 T \theta \hat{L}_{T}(z) \log p_{\rho}(z)\right\} .
$$

The proof of Proposition 7 is in Section 4.1. Think of $F_{T}$ as a fluctuation functional: $F_{T}\left(p_{\rho}\right)=o(T)$ as $T \rightarrow \infty$ because of (1.4), so in the r.h.s. of (2.9) the contribution of the expectation is of higher order than the prefactor. The point of Proposition 7 is that the prefactor has precisely the form we are looking for in (0.19). To complete the proof of (0.19), we must show that as $T \rightarrow \infty$ the expectation in (2.9) becomes independent of $x, y$ up to and including order 1 . This will be described in Sections 2.5-2.6.

\subsection{Separation of the time scales: Proposition 8}

Pick $0 \ll t \ll T$ and split the occupation time measure as

$$
\hat{L}_{T}=\frac{t}{T} \hat{L}_{t}+\frac{T-t}{T} \hat{L}_{t, T}
$$

where $\hat{L}_{t, T}$ is the occupation time measure over the time interval $[t, T)$. Later we shall let $T \rightarrow \infty$ followed by $t \rightarrow \infty$. The first limit will allow us to get $\hat{L}_{t, T}$ close to $p_{\rho}$, the second limit will allow us to get rid of the $x, y$-dependence.

Proposition 8 below separates the contributions from $\hat{L}_{t}$ and $\hat{L}_{t, T}$. We expand

$$
\begin{aligned}
F_{T}\left(\hat{L}_{T}\right) & =F_{T}\left(\frac{T-t}{T} \hat{L}_{t, T}+\frac{t}{T} \hat{L}_{t}\right) \\
& =F_{T}\left(\frac{T-t}{T} \hat{L}_{t, T}\right)+\int_{0}^{1} d \xi\left\langle\frac{t}{T} \hat{L}_{t}, D F_{T}\left[\frac{T-t}{T} \hat{L}_{t, T}+\xi \frac{t}{T} \hat{L}_{t}\right]\right\rangle .
\end{aligned}
$$


Here, $\langle\cdot, \cdot\rangle$ is the standard inner product and $D F_{T}$ is the Fréchet derivative of $F_{T}$ given by (see $(2.10)$ )

$$
D F_{T}[\lambda](z)=2 T H^{\prime}(2 T \lambda(z))-H(2 T)-2 T \theta \log p_{\rho}(z) .
$$

Using the identity $\sum_{z} \hat{L}_{t}(z)=1$, we may write

$$
\left\langle\frac{t}{T} \hat{L}_{t}, D F_{T}[\lambda]\right\rangle=2 t\left(H^{\prime}(2 T)-\frac{1}{2 T} H(2 T)+\left\langle\hat{L}_{t}, V_{T} \cdot \lambda+\theta \log \frac{\lambda}{p_{\rho}}\right\rangle\right)
$$

with $V_{T}: \mathbb{R}^{+} \rightarrow \mathbb{R}$ the potential

$$
V_{T}(\zeta)=H^{\prime}(2 T \zeta)-H^{\prime}(2 T)-\theta \log \zeta=\int_{2 T \zeta}^{2 T} \frac{d u}{u}\left[\theta-u H^{\prime \prime}(u)\right]
$$

and $V_{T} \cdot \lambda$ the composition of $V_{T}$ with $\lambda$. (The reason for splitting terms as in (2.14) is that $V_{T}$ is small for large $T$ (see $(0.15)$ ). Together with the trivial inclusions

$$
\begin{aligned}
& \left\{\hat{L}_{t, T} \in \mathcal{U}_{\epsilon_{1}}\left(p_{\rho}\right)\right\} \subseteq\left\{\hat{L}_{T} \in \mathcal{U}_{\epsilon}\left(p_{\rho}\right)\right\} \subseteq\left\{\hat{L}_{t, T} \in \mathcal{U}_{\epsilon_{2}}\left(p_{\rho}\right)\right\} \\
& \text { for } \epsilon_{1}=\frac{\epsilon-2 \delta}{1-\delta}, \epsilon_{2}=\frac{\epsilon+2 \delta}{1-\delta} \text { and } 0 \leq \frac{t}{T} \leq \delta
\end{aligned}
$$

valid when $0<\delta<\frac{\epsilon}{2}$, we obtain the following lower resp. upper bound for the expectation in the r.h.s. of (2.9).

Proposition 8 Fix $0<\delta<\frac{\epsilon}{2}$. Let $0 \leq t \leq T$ and $\epsilon_{i}(\delta, \epsilon)(i=1,2)$ be as in (2.16). Then for all $R>0$ and all $x, y \in \mathbb{Z}^{d}$

$$
\begin{aligned}
& E_{x, y}^{\rho}\left(\exp \left[F_{T}\left(\hat{L}_{T}\right)\right] \frac{1}{\sqrt{p_{\rho}\left(Z^{1}(T)\right) p_{\rho}\left(Z^{2}(T)\right)}} 1\left\{\hat{L}_{T} \in \mathcal{U}_{\epsilon}\left(p_{\rho}\right)\right\} 1\left\{\hat{\sigma}_{R}>t\right\}\right) \\
& \geq^{\geq^{(i=2)}} \quad \sum_{\tilde{x}, \tilde{y} \in T_{R}} P_{t}^{\rho}(x, \tilde{x}) P_{t}^{\rho}(y, \tilde{y}) E_{\tilde{x}, \tilde{y}}^{\rho}\left(\psi_{R}\left(x, y ; \tilde{x}, \tilde{y} ; \hat{L}_{T-t} ; t, T\right)\right. \\
& \left.\times \phi\left(Z^{1}(T-t), Z^{2}(T-t) ; \hat{L}_{T-t} ; t, T\right) 1\left\{\hat{L}_{T-t} \in \mathcal{U}_{\epsilon_{i}(\delta, \epsilon)}\left(p_{\rho}\right)\right\}\right) .
\end{aligned}
$$

Here $P_{t}^{\rho}(\cdot, \cdot)$ is the transition kernel of the random walk driven by $G_{\rho}$ in $(1.10)$, while $\psi_{R}$ and $\phi$ are the functions given by

$$
\begin{aligned}
& \psi_{R}(x, y ; \tilde{x}, \tilde{y} ; \mu ; t, T) \\
& =E_{x, y}^{\rho}\left(\exp \left[2 t \int_{0}^{1} d \xi\left\langle\hat{L}_{t}, V_{T} \cdot\left(\frac{T-t}{T} \mu+\xi \frac{t}{T} \hat{L}_{t}\right)+\theta \log \frac{\frac{T-t}{T} \mu+\xi \frac{t}{T} \hat{L}_{t}}{p_{\rho}}\right\rangle\right]\right. \\
& \left.\quad \times 1\left\{\operatorname{supp}\left(\hat{L}_{t}\right) \subseteq T_{R}\right\} \mid Z^{1}(t)=\tilde{x}, Z^{2}(t)=\tilde{y}\right)
\end{aligned}
$$

$\phi(\hat{x}, \hat{y} ; \mu ; t, T)$

$$
=\exp \left[2 t\left(H^{\prime}(2 T)-\frac{1}{2 T} H(2 T)\right)\right] \exp \left[F_{T}\left(\frac{T-t}{T} \mu\right)\right] \frac{1}{\sqrt{p_{\rho}(\hat{x}) p_{\rho}(\hat{y})}}
$$


for $0 \leq t \leq T, \mu \in \mathcal{P}\left(\mathbb{Z}^{d}\right)$ and $x, y, \tilde{x}, \tilde{y}, \hat{x}, \hat{y} \in \mathbb{Z}^{d}$.

The proof of Proposition 8 is in Section 4.2. The point of Proposition 8 is that the $x, y$-dependence sits all in the first three factors of the summand in (2.17).

\subsection{Loss of memory: Proof of Proposition 9}

Our last proposition shows that the first three factors of the summand in (2.17) become independent of $x, y$ for $T \rightarrow \infty$, and hence so does the expectation in the 1.h.s. of (2.17). The reason for this is that the transformed random walk has a drift towards 0 that increases rapidly with the distance to 0 , so it has strong ergodic properties.

Proposition 9 (1) For all $t \geq 0$, all $R>0$, all $0<\epsilon<\epsilon_{R}=\inf _{z \in T_{R}} p_{\rho}(z)$ and all $x, y \in \mathbb{Z}^{d}$

$$
\begin{aligned}
& \liminf _{T \rightarrow \infty} \inf _{\tilde{x}, \tilde{y} \in T_{R}} \inf _{\mu \in \mathcal{U}_{\epsilon}\left(p_{\rho}\right)} \psi_{R}(x, y ; \tilde{x}, \tilde{y} ; \mu ; t, T) \\
& \quad \geq\left(1-\frac{\epsilon}{\epsilon_{R}}\right)^{2 t \theta} \inf _{\tilde{x}, \tilde{y} \in T_{R}} P_{x, y}^{\rho}\left(\operatorname{supp}\left(\hat{L}_{t}\right) \subseteq T_{R} \mid Z^{1}(t)=\tilde{x}, Z^{2}(t)=\tilde{y}\right) \\
& \limsup _{T \rightarrow \infty} \sup _{\tilde{x}, \tilde{y} \in T_{R}} \sup _{\mu \in \mathcal{U}_{\epsilon}\left(p_{\rho}\right)} \psi_{R}(x, y ; \tilde{x}, \tilde{y} ; \mu ; t, T) \\
& \quad \leq\left(1+\frac{\epsilon}{\epsilon_{R}}\right)^{2 t \theta} .
\end{aligned}
$$

(2) For all $x \in \mathbb{Z}^{d}$

$$
\lim _{t \rightarrow \infty} \sup _{|\tilde{x}|=o(t / \log t)}\left|\frac{P_{t}^{\rho}(x, \tilde{x})}{P_{t}^{\rho}(0, \tilde{x})}-1\right|=0 .
$$

(3) For all $x, y \in \mathbb{Z}^{d}$

$$
\lim _{t \rightarrow \infty} \inf _{\substack{t / \log \log t=o(R) \\ R=o(t / \log t)}} \inf _{\tilde{x}, \tilde{y} \in T_{R}} P_{x, y}^{\rho}\left(\operatorname{supp}\left(\hat{L}_{t}\right) \subseteq T_{R} \mid Z^{1}(t)=\tilde{x}, Z^{2}(t)=\tilde{y}\right)=1 .
$$

The proof of Proposition 9 is in Section 4.3. We have now completed our list of key propositions.

\subsection{Completion of the proof of Theorem 3}

Let us finally collect Propositions 4-9 and explain why they prove Theorem 3. For this we take limits in the following order:

$$
T \rightarrow \infty, \delta \rightarrow 0, \epsilon \rightarrow 0, R=\sqrt{t}, t \rightarrow \infty .
$$


The summation in (2.5) is restricted to the box $T_{N}$ and the limit $N \rightarrow \infty$ is taken last. The proof comes in 4 steps.

1. Propositions 4-6 and (2.5) can be summarized as follows (the lower indices indicate the choice of the variables):

$$
\begin{aligned}
E_{x, y}\left(\exp \left[H \circ \hat{\ell}_{T}\right]\right)= & \left(1+a_{x, y, T, \epsilon}\right)\{\text { l.h.s. }(2.3)\}_{x, y, T, \epsilon} \\
\{\text { l.h.s. }(2.3)\}_{x, y, T, \epsilon}= & \sum_{z \in T_{N}}\{\text { l.h.s. }(2.6)\}_{x-z, y-z, T, \epsilon} \\
& +b_{N, x, y, T, \epsilon} E_{0,0}\left(\exp \left[H \circ \hat{\ell}_{T}\right]\right) \\
\{\text { l.h.s.(2.6) }\}_{x-z, y-z, T, \epsilon}= & \{\text { l.h.s. }(2.9)\}_{x-z, y-z, T, \epsilon, R, t} \\
& +c_{x-z, y-z, T, \epsilon, R, t} E_{x-z, y-z}\left(\exp \left[H \circ \hat{\ell}_{T}\right]\right)
\end{aligned}
$$

with

$$
\begin{array}{ll}
\lim _{T \rightarrow \infty} a_{x, y, T, \epsilon} & 0 \quad \begin{array}{l}
\text { for all } \epsilon>0 \text { and all } x, y \in \mathbb{Z}^{d} \\
\lim _{N \rightarrow \infty} b_{N, x, y, T, \epsilon}
\end{array}=0 \quad \begin{array}{l}
\text { uniformly in } T \geq t_{0} \text { and } 0<\epsilon \leq \epsilon_{0} \\
\text { for all } x, y \in \mathbb{Z}^{d}
\end{array}
\end{array}
$$

$\lim _{t \rightarrow \infty} \lim _{T \rightarrow \infty} c_{x, y, T, \epsilon, R=\sqrt{t}, t}=0 \quad$ uniformly in $0<\epsilon \leq \epsilon_{0}$ for all $x, y \in \mathbb{Z}^{d}$.

2. Propositions $7-9$ can be summarized as follows:

$$
\begin{aligned}
& \{\text { l.h.s. }(2.9)\}_{x-z, y-z, T, \epsilon, R, t} \quad=\quad \sqrt{p_{\rho}(x-z) p_{\rho}(y-z)} \\
& \times \exp [H(2 T)-\chi(\rho) 4 d \kappa T] \\
& \times\{\text { l.h.s. }(2.17)\}_{x-z, y-z, T, \epsilon, R, t} \\
& \{\text { l.h.s.(2.17) }\}_{x-z, y-z, T, \epsilon, R, t} \quad \geq^{(i=1)} \quad\{\text { r.h.s. }(2.17)\}_{x-z, y-z, T, \epsilon_{i}(\delta, \epsilon), R, t} \\
& \{\text { r.h.s.(2.17) }\}_{x-z, y-z, T, \epsilon_{i}(\delta, \epsilon), R, t}=\quad\left(1+d_{x-z, y-z, T, \delta, \epsilon, R, t}\right) \\
& \times\{\text { r.h.s. }(2.17)\}_{0,0, T, \epsilon_{i}(\delta, \epsilon), R, t}
\end{aligned}
$$

with

$\lim _{t \rightarrow \infty} \lim _{\epsilon \rightarrow 0} \lim _{\delta \rightarrow 0} \lim _{T \rightarrow \infty} d_{x, y, T, \delta, \epsilon, R=\sqrt{t, t}}=0 \quad$ for all $x, y \in \mathbb{Z}^{d}$.

3. Now first pick $x=y=0$. Then (2.23-2.24) and (2.25-2.26), together with the identity $E_{-z,-z}\left(\exp \left[H \circ \hat{\ell}_{T}\right]\right)=E_{0,0}\left(\exp \left[H \circ \hat{\ell}_{T}\right]\right)\left(z \in \mathbb{Z}^{d}\right)$ and the fact that $\lim _{\delta \rightarrow 0} \epsilon_{i}(\epsilon, \delta)=\epsilon$ $(i=1,2)$, yield a closed set of equations for $E_{0,0}\left(\exp \left[H \circ \hat{\ell}_{T}\right]\right)$ from which the expansion in (0.19) for $x=y=0$ easily follows. 
4. Finally, pick $x, y$ arbitrary. Then (2.23-2.24) and (2.25-2.26), together with the identity $E_{x-z, y-z}\left(\exp \left[H \circ \hat{\ell}_{T}\right]\right)=E_{x, y}\left(\exp \left[H \circ \hat{\ell}_{T}\right]\right)\left(z \in \mathbb{Z}^{d}\right)$ and the result in step 3 , yield the expansion in (0.19).

Note that the precise form of the higher order term $C_{2}(\rho, \kappa T)=o(T)$ in the exponent in (0.19) does not come out of the analysis. Clearly, it is sensitive to the precise form of $H$ beyond the asymptotics assumed in (0.15) (and remains hidden in the last factor in the r.h.s. of (2.25) after the limits in (2.22) are taken).

\section{Proof of Propositions 4-6}

\subsection{Proof of Proposition 4}

The difficulty behind the proof was explained in Section 2.1, as well as the route that is to be followed. We shall use several ideas from Bolthausen and Schmock (preprint 1994), where a similar problem is handled.

A key role will be played by the variational problem $(* *)$ and its restriction to $T_{N}=$ $(-N, N]^{d}$ with periodic boundary conditions (see Sections 0.4 and 5.3$)$. Let $\mathcal{M}$ resp. $\mathcal{M}^{N}$ denote the sets of minimizers of these variational problems. For $\epsilon>0$, define

$$
\begin{aligned}
& \mathcal{U}_{\epsilon}=\left\{\mu \in \mathcal{P}\left(\mathbb{Z}^{d}\right):\|\mu-\nu\|_{\ell^{1}}<\epsilon \text { for some } \nu \in \mathcal{M}\right\} \\
& \mathcal{U}_{\epsilon}^{N}=\left\{\mu \in \mathcal{P}\left(T_{N}\right):\|\mu-\nu\|_{\ell^{1}}<\epsilon \text { for some } \nu \in \mathcal{M}^{N}\right\}
\end{aligned}
$$

(see also (2.2)). We shall abbreviate

$$
\hat{P}_{x, y ; t}(\cdot)=\frac{E_{x, y}\left(1\{\cdot\} \exp \left[H \circ \hat{\ell}_{t}\right]\right)}{E_{x, y}\left(\exp \left[H \circ \hat{\ell}_{t}\right]\right)}
$$

and

$$
\begin{array}{lll}
\hat{L}_{t}(B) & =\sum_{z \in B} \hat{L}_{t}(z) & \left(B \subseteq \mathbb{Z}^{d}\right) \\
\hat{L}_{t}^{N}(B) & =\sum_{z \in B} \hat{L}_{t}^{N}(z) & \left(B \subseteq T_{N}\right),
\end{array}
$$

where $\hat{L}_{t}=\hat{\ell}_{t} / 2 t$ is the occupation time measure of the two random walks and $\hat{L}_{t}^{N}$ is its periodized version. The goal of this section is to prove that

$$
\limsup _{t \rightarrow \infty} \frac{1}{t} \log \hat{P}_{x, y ; t}\left(\hat{L}_{t} \notin \mathcal{U}_{\epsilon}\right)<0 \text { for all } \epsilon>0 \text { and } x, y \in \mathbb{Z}^{d} .
$$

This implies Proposition 4.

For ease of notation we shall drop the superscript. Keep in mind though that $P_{x, y ; t}$ and $L_{t}, L_{t}^{N}$ refer to two random walks. We now start the proof of (3.4). 
Proof. Fix $\epsilon>0$ and $x, y \in \mathbb{Z}^{d}$. Throughout the proof, $N$ is so large that $x, y \in T_{N}$. Define the event

$$
A_{t}^{N, \epsilon}=\bigcap_{z \in \mathbb{Z}^{d}}\left\{L_{t}\left(T_{N}+z\right) \leq 1-\frac{1}{4} \epsilon\right\}
$$

i.e., no translate of $T_{N}$ contains more than mass $1-\frac{1}{4} \epsilon$. We may then split

$$
\begin{aligned}
& P_{x, y ; t}\left(L_{t} \notin \mathcal{U}_{\epsilon}\right) \\
& \leq P_{x, y ; t}\left(L_{t} \notin \mathcal{U}_{\epsilon}, L_{t}^{N} \in \mathcal{U}_{\frac{1}{32 d} \epsilon}^{N}\right)+P_{x, y ; t}\left(L_{t}^{N} \notin \mathcal{U}_{\frac{1}{32 d} \epsilon}^{N}\right) \\
& \leq P_{x, y ; t}\left(L_{t} \notin \mathcal{U}_{\epsilon},\left[A_{t}^{N, \epsilon}\right]^{c}\right)+P_{x, y ; t}\left(L_{t}^{N} \in \mathcal{U}_{\frac{1}{32 d} \epsilon}^{N}, A_{t}^{N, \epsilon}\right)+P_{x, y ; t}\left(L_{t}^{N} \notin \mathcal{U}_{\frac{1}{32 d} \epsilon}^{N}\right) .
\end{aligned}
$$

In what follows we shall show that all three terms are exponentially small, which will prove (3.4). The proof comes in 7 steps.

1. Third term: By the full large deviation principle on $T_{N}$, there exists a $N_{0} \geq 1$ (depending on $\epsilon$ ) such that

$$
\limsup _{t \rightarrow \infty} \frac{1}{t} \log P_{x, y ; t}\left(L_{t}^{N} \notin \mathcal{U}_{\frac{1}{32 d} \epsilon}^{N}\right)<0 \text { for } N \geq N_{0} .
$$

Indeed, because of (3.2), this is a statement about a quotient of two terms, which behave resp. as

$$
\begin{gathered}
\exp \left[H(2 t)-\chi_{\frac{1}{32 d} \epsilon}^{N}(\rho) 4 d \kappa t+o(t)\right] \\
\exp [H(2 t)-\chi(\rho) 4 d \kappa t+o(t)] .
\end{gathered}
$$

Here $\chi(\rho)$ is given by $(* *)$, while

$$
\chi_{\epsilon}^{N}(\rho)=\frac{1}{2 d} \min _{p \notin \mathcal{U}_{\epsilon}^{N}} F_{d}(p) \quad\left(F_{d}=I_{d}+\rho J_{d}\right)
$$

(compare with (1.11-1.13) in Section 1.2). By Lemmas 16(f-g) in Section 5.3, we have $\chi_{\epsilon}^{N}(\rho)>\chi(\rho)$ for all $\epsilon>0$ and $N$ sufficiently large (depending on $\epsilon$ ). Together with (3.83.9) this implies (3.7).

2. First term: Note that

$$
\begin{aligned}
{\left[A_{t}^{N, \epsilon}\right]^{c} } & =\bigcup_{z \in \mathbb{Z}^{d}}\left\{L_{t}\left(T_{N}+z\right)>1-\frac{1}{4} \epsilon\right\} \\
& \subseteq \bigcup_{z \in \mathbb{Z}^{d}}\left\{\left\|L_{t}(\cdot+z)-L_{t}^{N}(\cdot)\right\|_{\ell^{1}}<\frac{1}{2} \epsilon\right\}
\end{aligned}
$$

(where elements of $\mathcal{P}\left(T_{N}\right)$ are viewed as elements of $\mathcal{P}\left(\mathbb{Z}^{d}\right)$ via the canonical embedding). Hence

$$
\left\{L_{t} \notin \mathcal{U}_{\epsilon},\left[A_{t}^{N, \epsilon}\right]^{c}\right\} \subseteq\left\{L_{t}^{N} \notin \mathcal{U}_{\frac{1}{2} \epsilon}\right\} \text { for all } N \geq 1 .
$$


Next, by Lemma $16(\mathrm{c})$ in Section 5.3 there exists a $N_{1} \geq 1$ (depending on $\epsilon$ ) such that

$$
\mathcal{U}_{\frac{1}{32 d} \epsilon}^{N} \subseteq \mathcal{U}_{\frac{1}{2} \epsilon} \quad \text { for all } N \geq N_{1}
$$

and hence

$$
\left\{L_{t}^{N} \notin \mathcal{U}_{\frac{1}{2} \epsilon}\right\} \subseteq\left\{L_{t}^{N} \notin \mathcal{U}_{\frac{1}{32 d} \epsilon}^{N}\right\} .
$$

So, combining (3.7), (3.12) and (3.14) we get

$$
\limsup _{t \rightarrow \infty} \frac{1}{t} \log P_{x, y ; t}\left(L_{t} \notin \mathcal{U}_{\epsilon},\left[A_{t}^{N, \epsilon}\right]^{c}\right)<0 \text { for all } N \geq N_{0} \vee N_{1} \text {. }
$$

3. Second term: We first write

$$
\begin{aligned}
P_{x, y ; t}\left(L_{t}^{N} \in \mathcal{U}_{\frac{1}{32 d} \epsilon}^{N}, A_{t}^{N, \epsilon}\right) & \leq \sum_{z \in T_{N}} P_{x, y ; t}\left(L_{t}^{N} \in \mathcal{U}_{\frac{1}{32 d} \epsilon}^{N}(z), A_{t}^{N, \epsilon}\right) \\
& =\sum_{z \in T_{N}} P_{x-z, y-z ; t}\left(L_{t}^{N} \in \mathcal{U}_{\frac{1}{32 d} \epsilon}^{N}(0), A_{t}^{N, \epsilon}\right) \\
& \leq\left|T_{N}\right| \max _{\substack{u, v \in T_{2 N} \\
u-v=x-y}} P_{u, v ; t}\left(L_{t}^{N} \in \mathcal{U}_{\frac{1}{32 d} \epsilon}^{N}(0), A_{t}^{N, \epsilon}\right),
\end{aligned}
$$

where $\mathcal{U}_{\epsilon}^{N}(z)$ denotes the $\epsilon$-neighborhood of the elements in $\mathcal{M}^{N}$ that are centered at $z$ (recall (3.1)). In the second line of (3.16) we have used that $A_{t}^{N, \epsilon}$ is shift invariant (recall (3.5)) and in the third line that $x, y \in T_{N}$. Next, put $N=5 M$ and define

$$
B_{t}^{5 M, \epsilon}=\bigcap_{z \in \mathbb{Z}^{d}}\left\{L_{t}\left(T_{5 M}+10 M z\right) \leq 1-\frac{1}{4} \epsilon\right\} \supseteq A_{t}^{5 M, \epsilon} .
$$

The proof of (3.4) will be complete once we show that

$$
\limsup _{t \rightarrow \infty} \frac{1}{t} \log \left[\max _{\substack{u, v \in T_{10 M} \\ u-v=x-y}} P_{u, v ; t}\left(L_{t}^{5 M} \in \mathcal{U}_{\frac{1}{32 d} \epsilon}^{5 M}(0), B_{t}^{5 M, \epsilon}\right)\right]<0 \text { for some } M \geq 1 .
$$

This will be done in steps $4-7$ below.

4. We begin with a combinatorial lemma. Define the halfspaces

$$
\begin{aligned}
& h_{k}^{i,+}=\left\{z \in \mathbb{Z}^{d}: z_{i}>(5+10 k) M\right\} \\
& h_{k}^{i,-}=\left\{z \in \mathbb{Z}^{d}: z_{i} \leq(5+10 k) M\right\} \quad(k \in \mathbb{Z}, i=1, \ldots, d) .
\end{aligned}
$$

Lemma $3 B_{t}^{5 M, \epsilon} \subseteq \bigcup_{k \in \mathbb{Z}} \bigcup_{i=1}^{d}\left\{L_{t}\left(h_{k}^{i,+}\right) \geq \frac{1}{8 d} \epsilon, L_{t}\left(h_{k}^{i,-}\right) \geq \frac{1}{8 d} \epsilon\right\}$

Proof. Put $\delta=\epsilon / 8 d$. We prove the inverse inclusion for the complements. Suppose that there is no $(k, i)$ such that $L_{t}\left(h_{k}^{i,+}\right) \geq \delta, L_{t}\left(h_{k}^{i,-}\right) \geq \delta$. Since for every $i$ there exists a $k(i)$ such that

$$
L_{t}\left(h_{k(i)-1}^{i,-}\right)<\delta \leq L_{t}\left(h_{k(i)}^{i,-}\right)
$$


it must be that $L_{t}\left(h_{k(i)}^{i,+}\right)<\delta$, and hence

$$
L_{t}\left(h_{k(i)-1}^{i,+} \cap h_{k(i)}^{i,-}\right)>1-2 \delta .
$$

Since $\cap_{i=1}^{d}\left[h_{z_{i}-1}^{i,+} \cap h_{z_{i}}^{i,-}\right]=T_{5 M}+10 M z$, it follows that

$$
L_{t}\left(T_{5 M}+10 M z\right)>1-2 d \delta \quad \text { for } z=(k(1), \ldots, k(d)) .
$$

5. Next, the random walks $Z^{1}, Z^{2}$ whose local times we are monitoring cannot move far away in time $t$, namely

$$
\lim _{t \rightarrow \infty} \frac{1}{t^{2}} \log \left[\max _{\substack{u, v \in T_{10 M} \\ u-v=x-y}} P_{u, v ; t}\left(Z^{i}(s) \notin T_{\left\lfloor t^{2}\right\rfloor} \text { for some } 0 \leq s \leq t\right)\right]<0 \quad(i=1,2) .
$$

Indeed, since $H \circ \hat{\ell}_{t} \leq H(2 t)=O(t \log t)=o\left(t^{2}\right)$, it suffices to prove the claim under the free random walk measure, i.e., without the exponential weight factor in (3.2). But the latter follows from a rough large deviation estimate because the jump times of the random walk are i.i.d. exponentially distributed with finite mean. The details are omitted. Combining (3.23) with Lemma 3, we see that in order to prove (3.18) it suffices to show that

$$
\begin{aligned}
\limsup _{t \rightarrow \infty} \frac{1}{t} \log \left[\max _{\substack{u, v \in T_{10 M} \\
u-v=x-y}} \sum_{|k| \leq \frac{t^{2}}{10 M}} \sum_{i=1}^{d}\right. \\
\left.P_{u, v ; t}\left(L_{t}^{5 M} \in \mathcal{U}_{\frac{1}{32 d} \epsilon}^{5 M}(0), L_{t}\left(h_{k}^{i,+}\right) \geq \frac{1}{8 d} \epsilon, L_{t}\left(h_{k}^{i,-}\right) \geq \frac{1}{8 d} \epsilon\right)\right]<0,
\end{aligned}
$$

which in turn is implied by

$$
\begin{aligned}
& \limsup _{t \rightarrow \infty} \frac{1}{t} \log \left[\sup _{\substack{u, v \in \mathbb{Z}^{d} \\
u-v=x-y}}\right. \\
& \left.\quad P_{u, v ; t}\left(L_{t}^{5 M} \in \mathcal{U}_{\frac{1}{32 d} \epsilon}^{5 M}(0), L_{t}\left(h^{+}\right) \geq \frac{1}{8 d} \epsilon, L_{t}\left(h^{-}\right) \geq \frac{1}{8 d} \epsilon\right)\right]<0
\end{aligned}
$$

with $h^{+}=h_{0}^{1,+}, h^{-}=h_{0}^{1,-}$. To go from (3.24) to (3.25) we have used that we may pick $k=0, i=1$ because of the shift-invariance and isotropy of the random walk and the shift-invariance of $H \circ \hat{\ell}_{t}$; the polynomial factor coming from counting the sum over $k, i$ is harmless.

6. Now, by Lemma $16(\mathrm{~b})$ in Section 5.3 there exists a $M_{0} \geq 1$ such that

$$
\left\{L_{t}^{5 M} \in \mathcal{U}_{\frac{1}{32 d} \epsilon}^{5 M}(0)\right\} \subseteq\left\{L_{t}^{5 M}\left(\text { int } T_{M}\right) \geq 1-\frac{1}{16 d} \epsilon\right\} \text { for } M \geq M_{0} .
$$


Hence to prove (3.25) it suffices to show that

$$
\begin{aligned}
& \limsup _{t \rightarrow \infty} \frac{1}{t} \log \left[\sup _{\substack{u, v \in \mathbb{Z}^{d} \\
u-v=x-y}}\right. \\
& \left.P_{u, v ; t}\left(L_{t}\left(h^{+}\right) \geq \frac{1}{8 d} \epsilon, L_{t}\left(h^{-}-4 M e_{1}\right) \geq \frac{1}{16 d} \epsilon, L_{t}^{5 M}\left(\operatorname{int} T_{M}\right) \geq 1-\frac{1}{16 d} \epsilon\right)\right]<0 .
\end{aligned}
$$

$\left(e_{1}=(1,0, \ldots, 0)\right)$. Indeed, by periodization with period $5 M$ the slab between $h^{+}$and $h^{-}-4 M e_{1}$ is mapped entirely inside $T_{5 M} \backslash T_{M}$. On the event in the r.h.s. of (3.26) this slab therefore carries mass at most $\frac{1}{16 d} \epsilon$. Consequently, on the event $\left\{L_{t}\left(h^{-}\right) \geq \frac{1}{8 d} \epsilon\right\}$ the half space $h^{-}-4 M e_{1}$ carries mass at least $\frac{1}{16 d} \epsilon$. What (3.27) says is that it is exponentially unlikely to have substantial local times in two halfspaces separated by a slab.

7. To prove (3.27) we shall do a reflection of the random walks w.r.t. the grid of size $2 M$. The object of this argument (see below) is to transfer the problem to the finite box $T_{5 M}$. Define

$$
\begin{aligned}
& g_{M} \quad=\bigcup_{k \in \mathbb{Z}} \bigcup_{i=1}^{d}\left\{z \in \mathbb{Z}^{d}: z_{i}=(2 k+1) M\right\} \\
& \sharp_{t}^{i}\left(g_{M}\right)=\frac{1}{t}\left|\left\{0 \leq s \leq t: Z^{i}(s) \in g_{M}, Z(s-) \notin g_{M}\right\}\right| \quad(i=1,2) \\
& \sharp_{t}\left(g_{M}\right)=\sharp_{t}^{1}\left(g_{M}\right)+\sharp_{t}^{2}\left(g_{M}\right),
\end{aligned}
$$

i.e., $t \sharp_{t}\left(g_{M}\right)$ counts the number of times the random walks hit $g_{M}$ during the time interval $[0, t]$. We may then bound the probability in (3.27) by the sum of two parts, namely for any $\delta>0$

$$
\begin{aligned}
& \text { (1) } P_{u, v ; t}\left(\sharp_{t}\left(g_{M}\right)>\delta, L_{t}\left(g_{M}\right) \leq \frac{1}{16 d} \epsilon\right) \\
& \text { (2) } P_{u, v ; t}\left(\sharp_{t}\left(g_{M}\right) \leq \delta, L_{t}\left(h^{+}\right) \geq \frac{1}{8 d} \epsilon, L_{t}\left(h^{-}-4 M e_{1}\right) \geq \frac{1}{16 d} \epsilon\right),
\end{aligned}
$$

where we use that $\left\{L_{t}^{5 M}\right.$ (int $\left.\left.T_{M}\right) \geq 1-\frac{1}{16 d} \epsilon\right\} \subseteq\left\{L_{t}\left(g_{M}\right) \leq \frac{1}{16 d} \epsilon\right\}$ because by periodization with period $5 M$ the grid $g_{M}$ is mapped entirely outside int $T_{M}$. Thus (3.27) will follow once we have proved Lemmas $4-5$ below.

Lemma 4 There exists a $C_{1}>0$ such that for all $\epsilon<C_{1} \delta$ and all $M \geq 1$

$$
\limsup _{t \rightarrow \infty} \frac{1}{t} \log \left[\sup _{\substack{u, v \in \mathbb{Z}^{d} \\ u-v=x-y}}(3.29)(1)\right]<0 .
$$

Proof. By shift-invariance and periodization with period $M$

$$
\sup _{\substack{u, v \in \mathbb{Z}^{d} \\ u-v=x-y}}(3.29)(1)=\max _{z \in T_{M}} P_{x-z, y-z, t}\left(\sharp_{t}\left(\partial T_{M}\right)>\delta, L_{t}^{M}\left(\partial T_{M}\right) \leq \frac{1}{16 d} \epsilon\right) .
$$


Therefore, similarly as in (3.7) and (3.8-3.9), the r.h.s. of (3.31) is a quotient of two terms. The denominator is the same as (3.9). Because $H \circ \hat{\ell}_{t} \leq H(2 t)$, the numerator can be bounded above by

$$
\exp [H(2 t)] \max _{z \in T_{M}} P_{x-z, y-z}\left(\sharp_{t}\left(\partial T_{M}\right)>\delta, L_{t}^{M}\left(\partial T_{M}\right) \leq \frac{1}{16 d} \epsilon\right),
$$

where in the r.h.s. of (3.32) appears the free random walk measure. Now, the latter probability equals

$$
\exp \left[-\zeta_{\delta, \epsilon}^{M}(\rho) 4 d \kappa t+o(t)\right]
$$

where $\zeta_{\delta, \epsilon}^{M}(\rho)$ can be made arbitrarily large by picking $\epsilon / \delta$ sufficiently small, uniformly in $M$. The reason is that it is unlikely for the random walks to spend a local time on $\partial T_{M}$ that is much smaller than $1 / 2 d \kappa$ times the number of times they hit $\partial T_{M}$. The details are omitted. Pick $\epsilon / \delta$ so small that $\zeta_{\delta, \epsilon}^{M}(\rho)>\chi(\rho)$ to get the claim.

Lemma 5 There exists a $C_{2}>0$ such that for all $\delta<C_{2} \epsilon \log (1 / \epsilon)$ and all $M$ sufficiently large (depending on $\delta, \epsilon$ )

$$
\limsup _{t \rightarrow \infty} \frac{1}{t} \log \left[\sup _{\substack{u, v \in \mathbb{Z}^{d} \\ u-v=x-y}}(3.29)(2)\right]<0 .
$$

Proof. The proof comes in 2 steps.

1. Consider the paths of the random walks up to time $t$. We can fold these paths inside $T_{5 M}$ by doing a number a reflections w.r.t. the hypersurfaces of dimension $d-1$ that lie on the grid $g_{M}$, starting from the outside and working our way inwards to $T_{5 M}$. With each reflection $H \circ \hat{\ell}_{t}$ increases, because $H$ is convex and because the local times of the paths are stacked on top of each other. Each piece of the paths that is thus folded adds a factor 2 to the counting. Hence we have

$$
\begin{aligned}
& \sup _{\substack{u, v \in \mathbb{Z} d \\
u-v=x-y}}(3.29)(2) \\
\leq & 2^{\delta t} \max _{z \in T_{5 M}} P_{x-z, y-z ; t}\left(L_{t}\left(T_{M}+4 M \epsilon_{1}\right) \geq \frac{1}{8 d} \epsilon, L_{t}\left(T_{M}\right) \geq \frac{1}{16 d}, L_{t}\left(T_{5 M}\right)=1\right) .
\end{aligned}
$$

Indeed, we can fold all the local time in $h^{+}$into the box $T_{M}+4 M e_{1}$, all the local time in $h^{-}-4 M e_{1}$ into the box $T_{M}$, and all the remaining local time in the box $T_{M}+2 M e_{1}$.

2. We now have an event inside the finite box $T_{5 M}$ where substantial local times are carried by two subboxes separated by a third box. The probability in (3.35) is the quotient of two terms, which behave resp. as (compare with (3.8-3.9))

$$
\begin{aligned}
& \exp \left[H(2 t)-\zeta_{\epsilon}^{M}(\rho) 4 d \kappa t+o(t)\right] \\
& \exp [H(2 t)-\chi(\rho) 4 d \kappa t+o(t)],
\end{aligned}
$$


where

$$
\zeta_{\epsilon}^{M}(\rho)=\min _{p \in \mathcal{C}(M, \epsilon)} F_{d}(p)
$$

with $\mathcal{C}(M, \epsilon)$ the set fitting the event in (3.35). Now, Lemma 16(h) in Section 5.3 shows that $\zeta_{\epsilon}^{M}(\rho)-\chi(\rho)>C_{2} \epsilon \log (1 / \epsilon)$ for some $C_{2}>0$ and $M$ sufficiently large (depending on $\epsilon$ ). Thus it suffices to pick $\delta$ smaller than this difference and the claim follows from (3.35).

By combining Lemmas $4-5$, picking $\epsilon$ so small that $\epsilon / C_{1}<C_{2} \epsilon \log (1 / \epsilon)$, and picking $\delta$ somewhere in the middle, we get (3.27). This completes the proof of Proposition 4.

\subsection{Proof of Proposition 5}

For $s \geq 0$ and $\Lambda \subseteq \mathbb{Z}^{d}$, let $\mathcal{P}_{s}(\Lambda)$ denote the set of all measures concentrated on $\Lambda$ with total mass $s$. For an arbitrary measure $\mu$ on $\mathbb{Z}^{d}$, write the abbreviation

$$
H \circ \mu=\sum_{z \in \mathbb{Z}^{d}} H(\mu(z)) .
$$

We recall that $(0.15)$ implies

$$
\lim _{t \rightarrow \infty}\left[H^{\prime}(\beta t)-H^{\prime}(\gamma t)\right]=\theta \log \left(\frac{\beta}{\gamma}\right) \text { for all } \beta>\gamma>0 .
$$

The following lemma, which is an estimate for one random walk, is the key to Proposition 5.

Lemma 6 Fix $\alpha>0$ arbitrarily and let $1>\beta>\gamma>0$ be such that

$$
\theta \log \left(\frac{\beta}{\gamma}\right)>4 d \kappa e^{\alpha}
$$

Let $\Lambda$ be a finite connected subset of $\mathbb{Z}^{d}$ containing 0 . Define

$$
\mathcal{A}=\mathcal{A}_{\beta, \gamma}(\Lambda)=\left\{\nu \in \mathcal{P}_{1}\left(\mathbb{Z}^{d}\right): \nu(0) \geq \beta, \min _{z \in \Lambda} \nu(z) \geq \gamma>\max _{z \in \Lambda^{c}} \nu(z)\right\} .
$$

(a) There exist $A>0$ and $T_{0}, R_{0}>0$ such that

$$
E_{x}\left(e^{H \circ \ell_{T}} 1\left\{\frac{1}{T} \ell_{T} \in \mathcal{A}\right\}\right) \leq A e^{-\alpha|x|} E_{0}\left(e^{H \circ \ell_{T}} 1\left\{\frac{1}{T} \ell_{T} \in \mathcal{A}\right\}\right)
$$

for all $T \geq T_{0}$ and all $x \notin T_{R_{0}}$.

(b) Let $\sigma=\inf \{s \geq 0: Z(s) \in \Lambda\}$ denote the first hitting time of $\Lambda$. Then there exist $A>0$ and $T_{0}, R_{0}>0$ such that

$$
\begin{aligned}
& E_{x}\left(e^{H \circ\left(\ell_{t}+\nu\right)} 1\left\{\frac{1}{T}\left(\ell_{t}+\nu\right) \in \mathcal{A}\right\} 1\{\sigma \leq t\} f\left(Z(t), \frac{1}{t} \ell_{t}\right)\right) \\
& \leq A e^{-\alpha|x|} E_{0}\left(e^{H \circ\left(\ell_{t}+\nu\right)} 1\left\{\frac{1}{T}\left(\ell_{t}+\nu\right) \in \mathcal{A}\right\} f\left(Z(t), \frac{1}{t} \ell_{t}\right)\right)
\end{aligned}
$$


for all $T \geq T_{0}$, all $0 \leq t \leq T_{0}$, all $x \notin T_{R_{0}}$, all $\nu \in \mathcal{P}_{T-t}\left(\mathbb{Z}^{d}\right)$, and all measurable functions $f: \mathbb{Z}^{d} \times \mathcal{P}_{1}\left(\mathbb{Z}^{d}\right) \rightarrow \mathbb{R}_{+}$satisfying

$$
f(z, p) \geq f(z, q) \text { whenever } p \geq q \text { on } \Lambda \text { and } p \leq q \text { on } \Lambda^{c} .
$$

Before presenting the proof of Lemma 6, let us give an heuristic explanation for (3.42). Let $Z$ be our random walk, starting at $x \notin \Lambda$ and hitting $\Lambda$ for the first time at time $\sigma$. The basic idea is to replace $(Z(s): s \in[0, \sigma])$ by a path that starts at 0 , stays at 0 during the time interval $[0, \sigma / 2]$ and moves to $Z(\sigma)$ during the time interval $(\sigma / 2, \sigma]$ without leaving $\Lambda$. In this way we switch from paths starting at $x$ to paths starting at 0 . In terms of local times this switch means that mass $\sigma / 2$ is moved from $\Lambda^{c}$ to 0 and another mass $\sigma / 2$ from $\Lambda^{c}$ to $\Lambda$. This moving obviously increases the event $\left\{\ell_{T} / T \in \mathcal{A}\right\}$. Moreover, we shall see that $H \circ \ell_{T}$ increases by at least $2 d \kappa e^{\alpha} \sigma$ because of (3.39-3.40). Hence we gain a factor $\exp \left[2 d \kappa e^{\alpha} \sigma\right]$ under the expectation. However, it will turn out that by the restriction to the new class of paths we loose a factor $C_{1} \exp [2 d \kappa \sigma]$. Altogether, we therefore gain a factor $\exp \left[2 d \kappa\left(e^{\alpha}-1\right) \sigma\right] / C_{1}$. But we shall see that

$$
C_{1} E_{x}\left(\exp \left[-2 d \kappa\left(e^{\alpha}-1\right) \sigma\right]\right) \leq C_{1} C_{2} e^{-\alpha|x|},
$$

which yields the desired prefactor in the r.h.s. of the first part of (3.42). The argument for (3.43) is essentially the same.

Proof. The proof of assertion (a) comes in 7 steps.

1. Choose $T_{0}$ so large that

$$
H^{\prime}(\beta T)-H^{\prime}(\gamma T) \geq 4 d \kappa e^{\alpha} \quad \text { for } T \geq T_{0} .
$$

This is possible because of (3.39-3.40). Throughout the proof, $T \geq T_{0}$ and $x \in \mathbb{Z}^{d}$ are fixed arbitrarily.

2. The monotonicity of $t \rightarrow H^{\prime}(t)$ obviously implies the following two inequalities:

$$
\begin{aligned}
& {[H(a+\Delta)+H(b)]-[H(a)+H(b+\Delta)]} \\
& \begin{cases}\geq 0 & \text { for } \Delta \geq 0, a \geq b \\
\geq \Delta\left[H^{\prime}(a)-H^{\prime}(b+\Delta)\right] & \text { for } \Delta \geq 0, a \geq b+\Delta .\end{cases}
\end{aligned}
$$

Using these inequalities we next prove the following statement:

$$
H \circ\left(\mu_{1}+\mu_{2}+\mu\right) \leq H \circ\left(\frac{s}{2} \delta_{0}+\mu_{3}+\mu\right)-4 d \kappa e^{\alpha} \frac{s}{2}
$$

for all

$$
0 \leq s \leq T, \mu_{1} \in \mathcal{P}_{\frac{s}{2}}\left(\Lambda^{c}\right), \mu_{2} \in \mathcal{P}_{\frac{s}{2}}\left(\Lambda^{c}\right), \mu_{3} \in \mathcal{P}_{\frac{s}{2}}(\Lambda), \mu \in \mathcal{P}_{T-s}\left(\mathbb{Z}^{d}\right)
$$

such that

$$
\frac{1}{T}\left(\mu_{1}+\mu_{2}+\mu\right) \in \mathcal{A} \text {. }
$$


Indeed, it follows from (3.50) and the definition of $\mathcal{A}$ in (3.41) that

$$
\max _{z \in \Lambda^{c}}\left(\mu_{1}+\mu_{2}+\mu\right)(z) \leq \min _{z \in \Lambda}\left(\mu_{1}+\mu_{2}+\mu\right)(z) .
$$

Hence, moving mass distribution $\mu_{2}$ from $\Lambda^{c}$ into $\Lambda$ and distributing it according to $\mu_{3}$, we can use the first part of (3.47) to estimate

$$
H \circ\left(\mu_{1}+\mu_{2}+\mu\right) \leq H \circ\left(\mu_{1}+\mu_{3}+\mu\right) .
$$

Moreover, after the move we obviously have

$$
\frac{1}{T}\left(\mu_{1}+\mu_{3}+\mu\right) \in \mathcal{A}
$$

SO

$$
\begin{aligned}
& \mu_{1}(0)+\mu_{3}(0)+\mu(0) \geq \beta T \\
& \max _{z \in \Lambda^{c}}\left(\mu_{1}+\mu_{3}+\mu\right)(z)<\gamma T .
\end{aligned}
$$

Therefore, now using the second part of (3.47), (3.54) and the monotonicity of $t \rightarrow H^{\prime}(t)$, we may move mass distribution $\mu_{1}$ from $\Lambda^{c}$ onto 0 , to obtain

$$
H \circ\left(\mu_{1}+\mu_{3}+\mu\right) \leq H \circ\left(\frac{s}{2} \delta_{0}+\mu_{3}+\mu\right)-\frac{s}{2}\left[H^{\prime}(\beta T)-H^{\prime}(\gamma T)\right] .
$$

Note that also after the last move

$$
\frac{1}{T}\left(\frac{s}{2} \delta_{0}+\mu_{3}+\mu\right) \in \mathcal{A} \text {. }
$$

Combining (3.46), (3.52) and (3.55), we arrive at (3.48).

3. We next use $(3.48-3.50)$ to move local times. Let

$$
\sigma=\inf \{u \geq 0: Z(u) \in \Lambda\}
$$

be the first hitting time of $\Lambda$. Clearly, $\ell_{T} / T \in \mathcal{A}$ implies $\sigma \leq T$ because $\beta>0$. To estimate the expectation in the 1.h.s. of (3.42) we proceed as follows. Applying the strong Markov property at time $\sigma$, we have

$$
E_{x}\left(e^{H \circ \ell_{T}} 1\left\{\frac{1}{T} \ell_{T} \in \mathcal{A}\right\}\right)=E_{x}\left(\psi\left(\sigma, Z(\sigma), \ell_{0, \sigma / 2}, \ell_{\sigma / 2, \sigma}\right) 1\{\sigma \leq T\}\right),
$$

where $\ell_{a, b}$ denotes the local time over the time interval $[a, b]$, and we define

$$
\psi\left(s, y, \mu_{1}, \mu_{2}\right)=E_{y}\left(e^{H \circ\left(\mu_{1}+\mu_{2}+\ell_{T-s}\right)} 1\left\{\frac{1}{T}\left(\mu_{1}+\mu_{2}+\ell_{T-s}\right) \in \mathcal{A}\right\}\right)
$$

for

$$
0 \leq s \leq T, y \in \Lambda, \mu_{1} \in \mathcal{P}_{\frac{s}{2}}\left(\Lambda^{c}\right), \mu_{2} \in \mathcal{P}_{\frac{s}{2}}\left(\Lambda^{c}\right)
$$


Since $\ell_{T-s} \in \mathcal{P}_{t-s}\left(\mathbb{Z}^{d}\right)$, we may now recall $(3.48-3.50)$ and (3.56) (for $\mu=\ell_{T-s}$ ) to estimate

$$
\psi\left(s, y, \mu_{1}, \mu_{2}\right) \leq \exp \left[-4 d \kappa e^{\alpha} \frac{s}{2}\right] \phi\left(s, y, \mu_{3}\right) \quad \text { for all } \mu_{3} \in \mathcal{P}_{\frac{s}{2}}(\Lambda)
$$

where we define

$$
\phi\left(s, y, \mu_{3}\right)=E_{y}\left(e^{H \circ\left(\frac{s}{2} \delta_{0}+\mu_{3}+\ell_{T-s}\right)} 1\left\{\frac{1}{T}\left(\frac{s}{2} \delta_{0}+\mu_{3}+\ell_{T-s}\right) \in \mathcal{A}\right\}\right) .
$$

Combining (3.58-3.62) we arrive at the bound

$$
\begin{aligned}
& E_{x}\left(e^{H \circ \ell_{T}} 1\left\{\frac{1}{T} \ell_{T} \in \mathcal{A}\right\}\right) \\
& \leq E_{x}\left(\exp \left[-4 d \kappa e^{\alpha} \frac{\sigma}{2}\right]\left(\min _{\nu \in \mathcal{P}_{\frac{\sigma}{2}}(\Lambda)} \phi(\sigma, Z(\sigma), \nu)\right) 1\{\sigma \leq T\}\right) .
\end{aligned}
$$

4. The 1.h.s. of (3.63) equals the 1.h.s. of (3.42). We next derive a lower bound for the r.h.s. of (3.42) that will be combined with (3.63) to yield (3.42). Let

$$
\tau=\inf \{u \geq 0: Z(u) \neq 0\}
$$

be the first exit time from 0 . For $y \in \Lambda$, define the set of paths

$$
B_{y}^{\frac{s}{2}}=\left\{Z(\cdot): Z(0)=0, Z\left(\frac{s}{2}\right)=y, Z(u) \in \Lambda \text { for } u \in\left[0, \frac{s}{2}\right]\right\} .
$$

Fix $0 \leq s \leq T$ and $y \in \Lambda$ arbitrarily. We may then apply the Markov property at time $s$ to write

$$
\begin{aligned}
& E_{0}\left(e^{H \circ \ell_{T}} 1\left\{\frac{1}{T} \ell_{T} \in \mathcal{A}\right\}\right) \geq E_{0}\left(1\left\{\tau>\frac{s}{2}, Z\left(\frac{s}{2}+\cdot\right) \in B_{y}^{\frac{s}{2}}\right\} e^{H \circ \ell_{T}} 1\left\{\frac{1}{T} \ell_{T} \in \mathcal{A}\right\}\right) \\
& =E_{0}\left(1\left\{\tau>\frac{s}{2}, Z\left(\frac{s}{2}+\cdot\right) \in B_{y}^{\frac{s}{2}}\right\} \phi\left(s, y, \ell_{\frac{s}{2}, s}\right)\right) .
\end{aligned}
$$

Here we have used that $\ell_{0, \frac{s}{2}}=\frac{s}{2} \delta_{0}$ on the event $\left\{\tau>\frac{s}{2}\right\}$ and $\ell_{\frac{s}{2}, s} \in \mathcal{P}_{\frac{s}{2}}(\Lambda)$ on the event $\left\{Z\left(\frac{s}{2}+\cdot\right) \in B_{y}^{\frac{s}{2}}\right\}$ (recall $\left.(3.62)\right)$. Since $P_{0}\left(\tau>\frac{s}{2}\right)=\exp [-d \kappa s]$, we thus find that

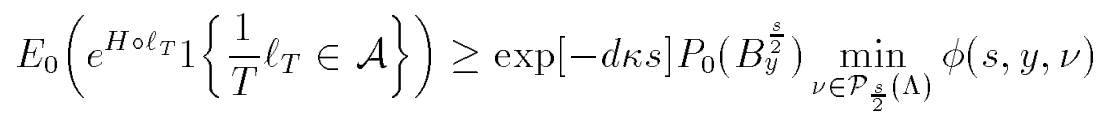

for all $0 \leq s \leq T$ and $y \in \Lambda$. Combining (3.63) and (3.67) we arrive at

$$
E_{x}\left(e^{H \circ \ell_{T}} 1\left\{\frac{1}{T} \ell_{T} \in \mathcal{A}\right\}\right) \leq K(x) E_{0}\left(e^{H \circ \ell_{T}} 1\left\{\frac{1}{T} \ell_{T} \in \mathcal{A}\right\}\right) .
$$

with

$$
K(x)=E_{x}\left(\left[\min _{y \in \partial \Lambda} P_{0}\left(B_{y}^{\frac{\sigma}{2}}\right)\right]^{-1} \exp \left[-2 d \kappa\left(2 e^{\alpha}-1\right) \frac{\sigma}{2}\right]\right) .
$$


Thus, to complete the proof of (3.42) we must show that $K(x) \leq A \exp (-\alpha|x|)$ for $x \notin T_{R_{0}}$ for some $A, R_{0}>0$.

5. We next estimate $\min _{y \in \partial \Lambda} P_{0}\left(B_{y}^{\frac{\sigma}{2}}\right)$ from below. Let $\tau_{1}, \tau_{2}, \ldots$ be the jump times of the random walk: i.i.d. exponentially distributed with mean $1 / 2 d \kappa$. Fix $y \in \partial \Lambda$ and let $D=D_{y}$ be the length of the shortest path from 0 to $y$ inside $\Lambda$. Obviously,

$$
\begin{aligned}
P_{0}\left(B_{y}^{s}\right) & \geq \frac{1}{(2 d)^{D}} P\left(\tau_{1}+\cdots+\tau_{D} \leq s<\tau_{1}+\cdots+\tau_{D}+\tau_{D+1}\right) \\
& =\frac{1}{(2 d)^{D}} \frac{(2 d \kappa s)^{D}}{D !} \exp [-2 d \kappa s] .
\end{aligned}
$$

From (3.70) it follows that there exists a $C_{1}>0$ such that

$$
\left[\min _{y \in \partial \Lambda} P_{0}\left(B_{y}^{s}\right)\right]^{-1} \leq C_{1} \exp [2 d \kappa s]\left\{1+(2 s)^{-D^{\prime}}\right\} \quad(s \geq 0)
$$

where $D^{\prime}=\sup _{y \in \Lambda} D_{y}$. Substitution into (3.69) gives

$$
K(x) \leq C_{1} E_{x}\left(\left\{1+\sigma^{-D^{\prime}}\right\} \exp \left[-2 d \kappa\left(e^{\alpha}-1\right) \sigma\right]\right) .
$$

We shall estimate the two terms in (3.72) separately.

6. Second term: To reach $\Lambda$ from $x$, the random walk $Z$ has to make at least $D^{\prime \prime}=\operatorname{dist}(x, \Lambda)$ jumps. Hence $\sigma \geq \tau_{1}+\cdots+\tau_{D^{\prime \prime}}$. Since $2 d \kappa\left(\tau_{1}+\cdots+\tau_{D^{\prime \prime}}\right)$ has a Gamma distribution with parameter $D^{\prime \prime}$, we can estimate for $D^{\prime \prime}>D^{\prime}$

$$
\begin{aligned}
& E_{x}\left(\sigma^{-D^{\prime}} \exp \left[-2 d \kappa\left(e^{\alpha}-1\right) \sigma\right]\right) \\
& \leq(2 d \kappa)^{D^{\prime}} \frac{1}{\left(D^{\prime \prime}-1\right) !} \int_{0}^{\infty} u^{D^{\prime \prime}-1-D^{\prime}} \exp \left[-e^{\alpha} u\right] d u \\
& =(2 d \kappa)^{D^{\prime}} \frac{\left(D^{\prime \prime}-1-D^{\prime}\right) !}{\left(D^{\prime \prime}-1\right) !} \exp \left[-\alpha\left(D^{\prime \prime}-D^{\prime}\right)\right] \\
& \leq C_{2} \exp \left[-\alpha\left(D^{\prime \prime}-D^{\prime}\right)\right]
\end{aligned}
$$

for some $C_{2}<\infty$. Clearly, $D^{\prime \prime} \geq|x|-C_{3}$ for some $C_{3}<\infty$.

7. First term: The same estimate with $D^{\prime}$ replaced by 0 . Combine steps 6 and 7 to get the bound on $K(x)$ claimed below (3.69). This completes the proof of assertion (a).

The proof of assertion (b) goes along the same lines. All we have to do is replace $\mu$ by $\mu+\nu \in \mathcal{P}_{T-s}\left(\mathbb{Z}^{d}\right)$ and $\ell_{T-s}$ by $\ell_{t-s}+\nu \in \mathcal{P}_{T-s}\left(\mathbb{Z}^{d}\right)$. Since $\frac{1}{T}\left(\ell_{t}+\nu\right) \in \mathcal{A}$ does not automatically imply $\sigma \leq t$, we need to include the indicator of the latter in the 1.h.s. of (3.43). The property of the function $f$ stated in (3.44) ensures that $f\left(Z(t), \frac{1}{t} \ell_{t}\right)$ can only increase when the path $(Z(s): s \in[0, \sigma])$ is redistributed inside $\Lambda$.

The next lemma is the analogue of Lemma 6 for two random walks. 
Lemma 7 Let the assumptions of Lemma 6 hold. Let $\sigma^{1}, \sigma^{2}$ denote the first hitting times of $\Lambda$. Then there exist $A>0$ and $T_{0}, R_{0}>0$ such that

$$
\begin{gathered}
E_{x, y}\left(e^{H \circ\left(\ell_{T}^{1}+\ell_{T}^{2}\right)} 1\left\{\frac{1}{2 T}\left(\ell_{T}^{1}+\ell_{T}^{2}\right) \in \mathcal{A}\right\} 1\left\{\sigma^{1} \leq t\right\} 1\left\{\sigma^{2} \leq t\right\}\right) \\
\leq A^{2} e^{-\alpha(|x|+|y|)} E_{0,0}\left(e^{H \circ\left(\ell_{T}^{1}+\ell_{T}^{2}\right)} 1\left\{\frac{1}{2 T}\left(\ell_{T}^{1}+\ell_{T}^{2}\right) \in \mathcal{A}\right\}\right)
\end{gathered}
$$

for all $T \geq T_{0}$ and all $x, y \notin T_{R_{0}}$.

Proof. This is an easy consequence of (3.43). Namely, first condition on $Z^{2}(\cdot)$, take the expectation over $Z^{1}(\cdot)$ by applying (3.43) with $\ell_{t}=\ell_{t}^{1}$ and $\nu=\ell_{T}^{2}$, and then take the expectation over $Z^{2}(\cdot)$. After that, interchange the order of the expectations (Fubini) and apply (3.43) with $\ell_{t}=\ell_{t}^{2}$ and $\nu=\ell_{T}^{1}$. Recall that $E_{x, y}=E_{x} \otimes E_{y}$.

We can now formulate the tightness result that implies Proposition 5. For $\mu \in \mathcal{P}_{1}\left(\mathbb{Z}^{d}\right)$, let

$$
\mathcal{U}_{\epsilon}(\mu)=\left\{\nu \in \mathcal{P}_{1}\left(\mathbb{Z}^{d}\right):\|\nu-\mu\|_{\ell^{1}}<\epsilon\right\}
$$

be the $\epsilon$-neighborhood of $\mu$ in the $\ell^{1}$-metric.

Lemma 8 Let $\mu \in \mathcal{P}_{1}\left(\mathbb{Z}^{d}\right)$ be such that

(i) $\mu(0)=\max _{z \in \mathbb{Z}^{d}} \mu(z)$

(ii) $\Lambda_{\gamma}=\left\{z \in \mathbb{Z}^{d}: \mu(z) \geq \gamma\right\}$ is connected for all $\gamma$ sufficiently small.

Fix $\alpha>0$ arbitrarily. Then there exist $A>0$ and $\epsilon_{0}, T_{0}, R_{0}>0$ (depending on $\mu, \alpha$ ) such that

$$
E_{x, y}\left(e^{H \circ\left(\ell_{T}^{1}+\ell_{T}^{2}\right)} 1\left\{\frac{1}{2 T}\left(\ell_{T}^{1}+\ell_{T}^{2}\right) \in \mathcal{U}_{\epsilon}(\mu)\right\}\right) \leq A^{2} e^{-\alpha(|x|+|y|)} E_{0,0}\left(e^{H \circ\left(\ell_{T}^{1}+\ell_{T}^{2}\right)}\right)
$$

for all $0<\epsilon \leq \epsilon_{0}$, all $T \geq T_{0}$ and all $x, y \notin T_{R_{0}}$.

Proof. Choose $\gamma_{0}>0$ so small that $\mu\left(\Lambda_{\gamma_{0}}\right)>\frac{1}{2}$ and

$$
\begin{aligned}
& \left(i^{\prime}\right) \theta \log \left(\frac{\mu(0)}{\gamma_{0}}\right)>4 d \kappa e^{\alpha} \\
& \left(i i^{\prime}\right) \Lambda=\Lambda_{\gamma} \text { is connected and contains } 0 \text { for all } 0<\gamma \leq \gamma_{0} .
\end{aligned}
$$

Next choose $1>\beta>\gamma>0$ such that assumption (3.40) of Lemma 6 is satisfied and

$$
\mu(0)>\beta, \min _{z \in \Lambda} \mu(z)>\gamma>\max _{z \in \Lambda^{c}} \mu(z) .
$$

(Because of (3.76)(i-ii), the latter can be done by picking $\beta<\mu(0)$ close to $\mu(0)$ and $\gamma<\gamma_{0}$ close to $\gamma_{0}$.) Now, because of (3.79) there exists $\epsilon_{0}>0$ such that for all $0<\epsilon \leq \epsilon_{0}$ and all $\tilde{\mu} \in \mathcal{U}_{\epsilon}(\mu)$

$$
\tilde{\mu}(0)>\beta, \min _{z \in \Lambda} \tilde{\mu}(z)>\gamma>\max _{z \in \Lambda^{c}} \tilde{\mu}(z) \text { and } \tilde{\mu}\left(\Lambda_{\gamma_{0}}\right)>\frac{1}{2} .
$$


Hence $\mathcal{U}_{\epsilon}(\mu) \subseteq \mathcal{A}$ for $0<\epsilon \leq \epsilon_{0}$, where $\mathcal{A}=\mathcal{A}_{\beta, \gamma}(\Lambda)$ with $\Lambda=\Lambda_{\gamma}$ the set defined in Lemma 6. Moreover, $\frac{1}{2 T}\left(\ell_{T}^{1}+\ell_{T}^{2}\right) \in \mathcal{U}_{\epsilon}(\mu)$ implies $\frac{1}{2 T}\left(\ell_{T}^{1}(\Lambda)+\ell_{T}^{2}(\Lambda)\right)>\frac{1}{2}$, which in turn implies $\ell_{T}^{1}(\Lambda)>0$ and $\ell_{T}^{2}(\Lambda)>0$, hence $\sigma^{1} \leq T$ and $\sigma^{2} \leq T$. We may therefore apply Lemma 7 (compare (3.41) with $(3.80)$ ) to obtain (3.77).

The proof of Proposition 5 is now complete. Indeed, we know from Theorem 2I(3)(ii) that the minimizer of $(* *)$ centered at 0 is unimodal in all directions, which guarantees that conditions $(3.76)(\mathrm{i}-\mathrm{ii})$ in Lemma 8 are fulfilled for $\mu=p_{\rho}=w_{\rho}^{2} /\left\|w_{\rho}\right\|_{\ell^{2}}^{2}$ (recall Section $0.5)$.

\subsection{Proof of Proposition 6}

The proof uses ideas from Section 3.2. The following lemma is an estimate for one random walk. Define

$$
\sigma_{R}=\inf \left\{s \geq 0: Z(s) \notin T_{R}\right\} .
$$

Let $\partial^{+} T_{R}$ denote the exterior boundary of $T_{R}$.

Lemma 9 Fix $x \in \mathbb{Z}^{d}$. Let the assumptions of Lemma 6 hold with $x \in \Lambda$. Let $\tau_{R}$ denote the first hitting time of $\Lambda$ after $\sigma_{R}$. Then there exist $A>0$ and $T_{0}, R_{0}, \delta_{0}>0$ such that

$$
E_{x}\left(e^{H \circ \ell_{T}} 1\left\{\frac{1}{T} \ell_{T} \in \mathcal{A}\right\} 1\left\{\sigma_{R} \leq t\right\}\right) \leq A^{2} e^{-2 \alpha R}\left|\partial^{+} T_{R}\right| t E_{x}\left(e^{H \circ \ell_{T}} 1\left\{\frac{1}{T} \ell_{T} \in \mathcal{A}\right\}\right)
$$

and

$$
\begin{array}{r}
E_{x}\left(e^{H \circ\left(\ell_{T}+\nu\right)} 1\left\{\frac{1}{2 T}\left(\ell_{T}+\nu\right) \in \mathcal{A}\right\} 1\left\{\sigma_{R} \leq t\right\} 1\left\{\tau_{R} \leq T\right\}\right) \\
\leq A^{2} e^{-2 \alpha R}\left|\partial^{+} T_{R}\right| t E_{x}\left(e^{H \circ\left(\ell_{T}+\nu\right)} 1\left\{\frac{1}{2 T}\left(\ell_{T}+\nu\right) \in \mathcal{A}\right\}\right)
\end{array}
$$

for all $t \geq 0$, all $R \geq R_{0}$, all $T \geq t \vee T_{0}$ with $t / T \leq \delta_{0}$ and all $\nu \in \mathcal{P}_{T}\left(\mathbb{Z}^{d}\right)$.

Proof. Throughout the proof we pick $R$ so large that $\Lambda \subseteq T_{R}$ and $x \in T_{R}$. We also pick $\delta_{0}=\beta$ and $t / T \leq \delta_{0}$. If $\ell_{T} / T \in \mathcal{A}$ and $\sigma_{R} \leq t$, then the latter guarantees that the random walk must hit 0 in the time interval $\left(\sigma_{R}, T\right)$ (recall (3.41)). We choose $T_{0}$ to be the same as in Lemma 6 . The proof of (3.82) comes in 8 steps.

1. First we use the strong Markov property at time $s$ write

$$
\begin{aligned}
& E_{x}\left(e^{H \circ \ell_{T}} 1\left\{\frac{1}{T} \ell_{T} \in \mathcal{A}\right\} 1\left\{\sigma_{R} \leq t\right\}\right) \\
& \quad=\sum_{z \in \partial+T_{R}} \int_{0}^{t} P_{x}\left(\sigma_{R} \in d s, Z(s)=z\right) E_{x}\left(\psi\left(s, z, \ell_{s}\right) \mid \sigma_{R}=s, Z(s)=z\right),
\end{aligned}
$$


where we define

$$
\psi(s, z, \mu)=E_{z}\left(e^{H \circ\left(\mu+\ell_{T-s}\right)} 1\left\{\frac{1}{T}\left(\mu+\ell_{T-s}\right) \in \mathcal{A}\right\}\right)
$$

for $0 \leq s \leq t, z \in \partial^{+} T_{R}$ and $\mu \in \mathcal{P}_{s}\left(T_{R}\right)$. Our choice of $\delta_{0}$ guarantees that $\frac{1}{T}\left(\mu+\ell_{T-s}\right) \in \mathcal{A}$ implies $\sigma \leq T-s$ for $s \in[0, t]$, where $\sigma$ again denotes the first hitting time of $\Lambda$.

2. By assertion (b) in Lemma 6 with $f \equiv 1$ we know that

$$
\psi(s, z, \mu) \leq A e^{-\alpha|z|} \psi(s, 0, \mu) \text { for all } 0 \leq s \leq t \text { and } \mu \in \mathcal{P}_{s}\left(T_{R}\right) .
$$

Combining this with (3.84) we have

$$
\begin{array}{r}
\text { l.h.s.(3.84) } \leq A e^{-\alpha R} \sum_{z \in \partial^{+} T_{R}} \int_{0}^{t} P_{x}\left(\sigma_{R} \in d s, Z(s)=z\right) \\
\quad \times E_{x}\left(\psi\left(s, 0, \ell_{s}\right) \mid \sigma_{R}=s, Z(s)=z\right) .
\end{array}
$$

3. Now apply Fubini to write

$$
E_{x}\left(\psi\left(s, 0, \ell_{s}\right) \mid \sigma_{R}=s, Z(s)=z\right)=E_{0}\left(\phi\left(s, x, z, \ell_{T-s}\right)\right),
$$

where we define

$$
\phi(s, x, z, \mu)=E_{x}\left(e^{H \circ\left(\mu+\ell_{s}\right)} 1\left\{\frac{1}{T}\left(\mu+\ell_{s}\right) \in \mathcal{A}\right\} \mid \sigma_{R}=s, Z(s)=z\right) .
$$

for $0 \leq s \leq t, z \in \partial^{+} T_{R}$ and $\mu \in \mathcal{P}_{T-s}\left(\mathbb{Z}^{d}\right)$.

4. Next, do a time reversal on the random walk over the time interval $[0, s]$. Let $z^{-}$be the unique site in $T_{R}$ that neighbors $z \in \partial^{+} T_{R}$. Then

$$
\begin{aligned}
& \phi(s, x, z, \mu) \\
& \quad=\frac{1}{2 d} E_{z^{-}}\left(e^{H \circ\left(\mu+\ell_{s}\right)} 1\left\{\frac{1}{T}\left(\mu+\ell_{s}\right) \in \mathcal{A}\right\} \mid \sigma_{R}>s, Z(s)=x, Z(s+) \neq x\right) \\
& P_{x}\left(\sigma_{R} \in d s, Z(s)=z\right) \\
& \quad=\frac{1}{2 d} P_{z^{-}}\left(\sigma_{R}>s, Z(s)=x\right) 2 d \kappa d s .
\end{aligned}
$$

Here the jump away from $z$ to $z^{-}$at time $s$ is replaced by a jump away from $x$ at time $s$ in the time reversed random walk. The factor $2 d$ counts the number of ways this last jump can occur. The local times are invariant under the time reversal.

5. Combining (3.87-3.90) we obtain

$$
\text { l.h.s.(3.84) } \leq A e^{-\alpha R} \sum_{z \in \partial^{+} T_{R}} \int_{0}^{t} 2 d \kappa d s P_{z^{-}}\left(\sigma_{R}>s, Z(s)=x\right)
$$




$$
\times E_{0}\left(\left.E_{z^{-}}\left(e^{H \circ\left(\mu+\ell_{s}\right)} 1\left\{\frac{1}{T}\left(\mu+\ell_{s}\right) \in \mathcal{A}\right\} \mid \sigma_{R}>s, Z(s)=x, Z(s+) \neq x\right)\right|_{\mu=\ell_{T-s}}\right) .
$$

6. Again apply Fubini. After that we can write

$$
\text { r.h.s. }(3.91)=A e^{-\alpha R} \sum_{z \in \partial^{+} T_{R}} \int_{0}^{t} d s E_{0}\left(\xi\left(s, x, z^{-}, \ell_{T-s}\right)\right) \text {, }
$$

where we define

$$
\xi\left(s, x, z^{-}, \mu\right)=E_{z^{-}}\left(e^{H \circ\left(\mu+\ell_{s}\right)} 1\left\{\frac{1}{T}\left(\mu+\ell_{s}\right) \in \mathcal{A}\right\} 1\left\{\sigma_{R}>s, Z(s)=x\right\}\right) .
$$

7. Next, $Z(s)=x$ implies $\sigma \leq s$ because $x \in \Lambda$. We may therefore apply assertion (b) in Lemma 6 with $f(z, p)=\delta_{x}(z) 1\left\{p\left(T_{R}\right)=1\right\}$, to obtain

$$
\xi\left(s, x, z^{-}, \mu\right) \leq A e^{-\alpha R} \xi(s, x, 0, \mu) .
$$

Combining (3.91-3.94) we arrive at

$$
\text { l.h.s.(3.84) } \leq A^{2} e^{-2 \alpha R} \sum_{z \in \partial+T_{R}} \int_{0}^{t} d s E_{0}\left(\xi\left(s, x, 0, \ell_{T-s}\right)\right) \text {. }
$$

However, using the strong Markov property at time $s$ and doing once more a time reversal of the random walk over the time interval $[0, s]$, we may write

$$
E_{0}\left(\xi\left(s, x, 0, \ell_{T-s}\right)\right)=E_{x}\left(e^{H \circ \ell_{T}} 1\left\{\frac{1}{T} \ell_{T} \in \mathcal{A}\right\} 1\left\{\sigma_{R}>s, Z(s)=0\right\}\right) .
$$

8. Finally, drop the last indicator to get

$$
\text { l.h.s.(3.84) } \leq A^{2} e^{-2 \alpha R}\left|\partial^{+} T_{R}\right| t E_{x}\left(e^{H \circ \ell_{T}} 1\left\{\frac{1}{T} \ell_{T} \in \mathcal{A}\right\}\right) .
$$

This completes the proof of (3.82).

The proof of (3.83) goes along the same lines. (Compare with the proof of assertion (b) in Lemma 6.)

The analogue of Lemma 9 for two random walks is similar. Namely, using (3.83) we get the estimate

$$
\begin{aligned}
& E_{x, y}\left(e^{H \circ\left(\ell_{T}^{1}+\ell_{T}^{2}\right)} 1\left\{\frac{1}{2 T}\left(\ell_{T}^{1}+\ell_{T}^{2}\right) \in \mathcal{A}\right\}\right. \\
& \left.\times\left[1\left\{\sigma_{R}^{1} \leq t\right\} 1\left\{\tau_{R}^{1} \leq T\right\}+1\left\{\sigma_{R}^{2} \leq t\right\} 1\left\{\tau_{R}^{2} \leq T\right\}\right]\right) \\
& \quad \leq 2 A^{2} e^{-2 \alpha R}\left|\partial^{+} T_{R}\right| t E_{x, y}\left(e^{H \circ\left(\ell_{T}^{1}+\ell_{T}^{2}\right)} 1\left\{\frac{1}{2 T}\left(\ell_{T}^{1}+\ell_{T}^{2}\right) \in \mathcal{A}\right\}\right)
\end{aligned}
$$

(compare with the proof of Lemma 7). 
For the final step in the proof of Proposition 6 , we recall that $\mathcal{U}_{\epsilon}\left(p_{\rho}\right) \subseteq \mathcal{A}$ for $0<\epsilon \leq \epsilon_{0}$ (see the proof of Lemma 8) and that $\hat{\sigma}=\min \left\{\sigma_{R}^{1}, \sigma_{R}^{2}\right\}$ is the stopping time defined in (2.7). We choose $\gamma_{0}$ so large that

$$
p_{\rho}\left(\Lambda_{\gamma_{0}}\right)>\frac{1}{2}\left(1+\delta_{0}\right)
$$

(recall that $\delta_{0}=\beta<1$ ). Then the same inequality holds for all measures in $\mathcal{U}_{\epsilon}\left(p_{\rho}\right)$, provided $\epsilon \leq \epsilon_{0}$ and $\epsilon_{0}$ is sufficiently small. But now we note that

$$
\frac{1}{2 T}\left(\ell_{T}^{1}+\ell_{T}^{2}\right) \in \mathcal{U}_{\epsilon}\left(p_{\rho}\right), \frac{t}{T} \leq \delta_{0}, \sigma_{R}^{i} \leq t \Longrightarrow \tau_{R}^{i} \leq T \quad(i=1,2) .
$$

Hence we can apply (3.83) and get the claim in Proposition 6.

\section{Proof of Propositions 7-9}

\subsection{Proof of Proposition 7}

Let $u_{\rho}^{2}=p_{\rho}=w_{\rho}^{2} /\left\|w_{\rho}\right\|_{\ell^{2}}^{2}=\left(v_{\rho} /\left\|v_{\rho}\right\|_{\ell^{2}}\right)^{\otimes d}$ be the unique centered minimizer of $(* *)$ in Section 0.4. To ease the notation we shall write $u$ instead of $u_{\rho}$.

Lemma 10 The semigroup $S_{p}=\left(S_{p}(t): t \geq 0\right)$ associated with the generator $G_{p}$ in (1.10) is given by

$$
\left(S_{\rho}(t) f\right)(x)=\frac{1}{u(x)} E_{x}\left(\exp \left[-\int_{0}^{t} d s \kappa \frac{\Delta u}{u}(Z(s))\right] u(Z(t)) f(Z(t))\right)
$$

and is a strongly continuous contraction semigroup on $\ell^{2}\left(\mathbb{Z}^{d} ; u^{2}\right)$.

Proof. Elementary. The r.h.s. of (4.1) is well defined because $u$ is strictly positive everywhere (see Lemma 13 in Section 5.1) and $(\Delta u) / u$ is bounded from below (see (4.6) below). The semigroup $S=(S(t): t \geq 0)$ associated with $\kappa \Delta$ (the generator of our reference random walk) is given by $(S(t) f)(x)=E_{x}(f(Z(t)))$ and is a strongly continuous contraction semigroup on $\ell^{2}\left(\mathbb{Z}^{d}\right)$. We compute with the help of (4.1)

$$
\begin{aligned}
& \left(G_{\rho} f\right)(x)=\lim _{t \downarrow 0} \frac{1}{t}\left(S_{\rho}(t) f-f\right)(x) \\
& =\frac{1}{u(x)}\left\{-\kappa(\Delta u)(x) f(x)+\lim _{t \downarrow 0} \frac{1}{t}(S(t)[u f]-[u f])(x)\right\} \\
& =\frac{1}{u(x)}\{-\kappa(\Delta u)(x) f(x)+\kappa \Delta(u f)(x)\} \\
& =\frac{1}{u(x)} \kappa \sum_{y:|y-x|=1}\{-[u(y)-u(x)] f(x)+[u(y) f(y)-u(x) f(x)]\} \\
& =\frac{1}{u(x)} \kappa \sum_{y:|y-x|=1} u(y)[f(y)-f(x)] .
\end{aligned}
$$


Indeed, this coincides with (1.10). Next, the semigroup property $S_{\rho}(s+t)=S_{\rho}(s) S_{\rho}(t)$ follows from (4.1) by using the Markov property of the reference random walk at time $s$. The strong continuity of $S_{p}$ follows from the strong continuity of $S$ and the boundedness of the exponential in (4.1). The contraction property of $S_{p}$ follows from the inequality

$$
\left\langle f, G_{\rho} f\right\rangle_{\ell^{2}\left(\mathbb{Z}^{d} ; u^{2}\right)}=-\sum_{\{x, y\}:|x-y|=1} u(x) u(y)[f(x)-f(y)]^{2} \leq 0 .
$$

The above representation leads us to the following.

Lemma 11 Let $P_{x, y}=P_{x} \otimes P_{y}$ and $P_{x, y}^{\rho}=P_{x}^{\rho} \otimes P_{y}^{\rho}$. Then for any $T \geq 0$

$$
\begin{aligned}
& \frac{d P_{x, y}^{\rho}}{d P_{x, y}}\left(\left(Z^{1}(s), Z^{2}(s)\right)_{s \in[0, T]}\right) \\
& \quad=\frac{u\left(Z^{1}(T)\right) u\left(Z^{2}(T)\right)}{u(x) u(y)} \exp \left[-\int_{0}^{T} d s \kappa\left\{\frac{\Delta u}{u}\left(Z^{1}(s)\right)+\frac{\Delta u}{u}\left(Z^{2}(s)\right)\right\}\right] .
\end{aligned}
$$

Proof. Immediate from (4.1).

Using Lemma 11 we can now do the absolute continuous transformation in the expectation appearing in the 1.h.s. of (2.9) in Proposition 7. Indeed, recalling that $\ell_{T}^{i}(x)=$ $\int_{0}^{T} d s 1_{\left\{Z^{i}(s)=x\right\}}(i=1,2)$, we obtain

$$
\begin{aligned}
E_{x, y}\left(\exp \left[H \circ \hat{\ell}_{T}\right] 1\left\{\frac{1}{2 T} \hat{\ell}_{T} \in \mathcal{U}_{\epsilon}\left(p_{\rho}\right)\right\} 1\left\{\hat{\sigma}_{R}>t\right\}\right) \\
=u(x) u(y) E_{x, y}^{\rho}\left(\exp \left[H \circ \hat{\ell}_{T}\right] \exp \left[\sum_{z} \hat{\ell}_{T}(z)\left\{\kappa \frac{\Delta u}{u}(z)\right\}\right]\right. \\
\left.\times \frac{1}{u\left(Z^{1}(T)\right) u\left(Z^{2}(T)\right)} 1\left\{\frac{1}{2 T} \hat{\ell}_{T} \in \mathcal{U}_{\epsilon}\left(p_{\rho}\right)\right\} 1\left\{\hat{\sigma}_{R}>t\right\}\right) .
\end{aligned}
$$

To complete the proof of Proposition 7, we simply note that

$$
\frac{\Delta u}{u}(z)=-2 \rho \log u(z)-2 d \chi(\rho)
$$

as follows from (*) in Section 0.3 and Proposition 3 via the relation $u=\left(v_{\rho} /\left\|v_{\rho}\right\|_{\ell^{2}}\right)^{\otimes d}$. After substituting (4.6) into the r.h.s. of (4.5) and using the relations $u^{2}=p_{\rho}, \rho=\theta / \kappa$, $\hat{L}_{T}=\hat{\ell}_{T} / 2 T$ and $\sum_{z} \hat{L}_{T}(z)=1$, we obtain the r.h.s. of $(2.9)$.

We conclude this section with the following observation.

Lemma 12 The random walk driven by $G_{\rho}$ is ergodic with $u^{2}$ as the reversible equilibrium. 
Proof. Elementary. To prove that $u^{2}$ is a reversible equilibrium, we compute for any $f, g$ with the help of $(1.10)$

$$
\begin{aligned}
\sum_{x}[u(x)]^{2} f(x)\left(G_{\rho} g\right)(x) & =\sum_{x} \sum_{y:|y-x|=1} \kappa u(x) u(y) f(x)[g(y)-g(x)] \\
& =\sum_{y} \sum_{x:|x-y|=1} \kappa u(x) u(y) g(y)[f(x)-f(y)] \\
& =\sum_{y}[u(y)]^{2} g(y)\left(G_{\rho} f\right)(y) .
\end{aligned}
$$

The ergodicity of the transition probabilities immediately follows from (4.10) and (4.12) below, which makes that $u^{2}$ is the unique equilibrium.

\subsection{Proof of Proposition 8}

Proof. Consider the 1.h.s. of (2.17). First bound $1\left\{\hat{L}_{T} \in \mathcal{U}_{\epsilon}\left(p_{\rho}\right)\right\}$ from below and above by $1\left\{\hat{L}_{t, T} \in \mathcal{U}_{\epsilon_{1}(\delta, \epsilon)}\left(p_{\rho}\right)\right\}$ resp. $1\left\{\hat{L}_{t, T} \in \mathcal{U}_{\epsilon_{2}(\delta, \epsilon)}\left(p_{\rho}\right)\right\}$ using (2.16). Next substitute (2.12), as

well as (2.14) with $\lambda=\frac{T-t}{T} \mu+\frac{t}{T} \hat{L}_{t}$ and $\mu=\hat{L}_{t, T}$, and write $\left\{\hat{\sigma}_{R}>t\right\}=\left\{\operatorname{supp}\left(\hat{L}_{t}\right) \subseteq T_{R}\right\}$. Next, let $\mathcal{F}_{t, T}$ denote the $\sigma$-field generated by the two random walks on the time interval $[t, T]$. We can take the conditional expectation over the two random walks on the time interval $[0, t]$ given $\mathcal{F}_{t, T}$. Since $\hat{L}_{t, T}$ is $\mathcal{F}_{t, T}$-measurable, this produces the two transition kernels as well as the product under the expectation in the r.h.s. of (2.17). Finally, take the expectation over $\mathcal{F}_{t, T}$ using the Markov property at time $t$ and shift $[t, T]$ to $[0, T-t]$.

\subsection{Proof of Proposition 9}

Proof. (1) Fix $t \geq 0$ and $R>0$. Note first that $\epsilon_{R}>0$ because $p_{\rho}>0$ everywhere (see Lemma 13 in Section 5.1). Next, we have $\lim _{T \rightarrow \infty} V_{T}\left(\zeta_{T}\right)=0$ as long as $\zeta_{T}$ is bounded away from 0 and $\infty$. This easily follows from (0.15) and (2.15). Next, if $0<\epsilon<\epsilon_{R}$ then $\mu \in \mathcal{U}_{\epsilon}\left(p_{\rho}\right)$ guarantees that $\inf _{z \in T_{R}} \mu(z)>0$. Together with $\operatorname{supp}\left(\hat{L}_{t}\right) \subseteq T_{R}$ we therefore have that, for $t$ fixed and $T \rightarrow \infty$, the first part of the inner product in the definition of $\psi_{R}(x, y ; \tilde{x}, \tilde{y} ; \mu ; t, T)$ in (2.18) vanishes uniformly in $\tilde{x}, \tilde{y} \in T_{R}$ and $\mu \in \mathcal{U}_{\epsilon}\left(p_{\rho}\right)$. The bounds in (2.19) are now easily obtained from the second part of the inner product by using that $\left|\mu(z)-p_{\rho}(z)\right|<\epsilon$ for all $z \in \mathbb{Z}^{d}$ when $\mu \in \mathcal{U}_{\epsilon}\left(p_{\rho}\right)$.

(2) By (4.1) and (4.6)

$$
\begin{aligned}
P_{t}^{\rho}(x, \tilde{x}) & =\left(S_{\rho}(t) \delta_{\tilde{x}}\right)(x) \\
& =\frac{u(\tilde{x})}{u(x)} E_{x}\left(\exp \left[-\int_{0}^{t} \kappa \frac{\Delta u}{u}(Z(s)) d s\right] 1\{Z(t)=\tilde{x}\}\right) \\
& =\frac{u(\tilde{x})}{u(x)} E_{x}\left(\exp \left[-\int_{0}^{t} V(Z(s)) d s\right] 1\{Z(t)=\tilde{x}\}\right)
\end{aligned}
$$


with $V: \mathbb{Z}^{d} \rightarrow \mathbb{R}$ the potential (recall (4.6) and Proposition 3)

$$
V(x)=2 \theta \log u(x)+2 d \kappa \chi(\rho)=2 \theta \sum_{i=1}^{d} \log v_{\rho}\left(x^{i}\right) .
$$

Now, let $\left(S_{V}(t): t \geq 0\right)$ be the semigroup associated with the generator $G_{V}=\kappa \Delta+V$. Then, using the Feynman-Kac formula, we have

$$
P_{t}^{\rho}(x, \tilde{x})=\frac{u(\tilde{x})}{u(x)}\left(S_{V}(t) \delta_{\tilde{x}}\right)(x)=\frac{u(\tilde{x})}{u(x)}\left\langle\delta_{x}, S_{V}(t) \delta_{\tilde{x}}\right\rangle,
$$

and so

$$
\frac{P_{t}^{\rho}(x, \tilde{x})}{P_{t}^{\rho}(y, \tilde{x})}=\frac{\frac{1}{u(x)}\left\langle\delta_{x}, S_{V}(t) \delta_{\tilde{x}}\right\rangle}{\frac{1}{u(y)}\left\langle\delta_{y}, S_{V}(t) \delta_{\tilde{x}}\right\rangle}
$$

with $\langle\cdot, \cdot\rangle$ the standard inner product.

The generator $G_{V}$ is self-adjoint and $G_{V} u=0$. Because $V$ is bounded from above and $\lim _{|x| \rightarrow \infty} V(x)=-\infty$, we know that $G_{V}$ has a compact resolvent $R(\lambda)=\left(\lambda-G_{V}\right)^{-1}$ in $\ell^{2}\left(\mathbb{Z}^{d}\right)$. From the semigroup representation of $R(\lambda)$ (which holds for $\lambda$ sufficiently large) it is also clear that $R(\lambda)$ is a positive operator. Therefore, by the strict positivity of $u$, we see that 0 is the largest eigenvalue of $G_{V}$ and that this eigenvalue is simple. Moreover, the compactness of $R(\lambda)$ tells us that the rest of the spectrum lies in $\left(-\infty,-\lambda_{0}\right]$ for some $\lambda_{0}>0$, the spectral gap.

Next, let $\Pi$ denote the projection onto $u$, i.e., $\Pi f=\langle u, f\rangle u /\langle u, u\rangle$. Then, by the spectral theorem, we have

$$
\begin{aligned}
\left\langle\delta_{x}, S_{V}(t) \delta_{\tilde{x}}\right\rangle & =\left\langle\delta_{x}, \Pi \delta_{\tilde{x}}\right\rangle+\left\langle\delta_{x},\left[S_{V}(t)-\Pi\right] \delta_{\tilde{x}}\right\rangle \\
& =\frac{u(x) u(\tilde{x})}{\langle u, u\rangle}+O\left(e^{-\lambda_{0} t}\right) \quad(t \rightarrow \infty) .
\end{aligned}
$$

Combining (4.11-4.12) we find

$$
\frac{P_{t}^{\rho}(x, \tilde{x})}{P_{t}^{\rho}(y, \tilde{x})}=\frac{1+\frac{\langle u, u\rangle}{u(x)} O\left(\frac{1}{u(\tilde{x})} e^{-\lambda_{0} t}\right)}{1+\frac{\langle u, u\rangle}{u(y)} O\left(\frac{1}{u(\tilde{x})} e^{-\lambda_{0} t}\right)} \quad(t \rightarrow \infty) .
$$

Thus the ratio tends to 1 when the order term tends to zero. But, by $(0.14)$ and the fact that $u(\tilde{x})=\left[\prod_{i=1}^{d} v_{\rho}\left(\tilde{x}_{i}\right)\right] / \exp [d \chi(\rho) / \rho]$ (recall Proposition 3$)$, this will be the case when $\left|\tilde{x}^{i}\right| \log \left|\tilde{x}^{i}\right|=o(t)$ for $i=1, \ldots, d$. Hence we have proved the claim in $(2.20)$.

(3) Because of the product property of the transition kernel

$$
P_{t}^{\rho, d}(x, y)=\prod_{i=1}^{d} P_{t}^{\rho, 1}\left(x^{i}, y^{i}\right) \text { for all } x, y \in \mathbb{Z}^{d},
$$


it suffices to give the proof of (2.21) for $d=1$. Moreover, because the two random walks are independent, it suffices to prove the analogue statement for one random walk. Thus, letting

$$
\sigma_{R}=\inf \{s \geq 0: Z(s) \notin[-R, R]\},
$$

we must show that

$$
\lim _{t \rightarrow \infty} \inf _{\substack{t / \log \log t=o(R) \\ R=o(t / \log t)}} \inf _{\tilde{x} \in[-R, R]} P_{x}^{\rho}\left(\sigma_{R}>t \mid Z(t)=\tilde{x}\right)=1 .
$$

Fix $x \in \mathbb{Z}$ and $\tilde{x} \in[-R, R]$. By time reversal we have

$$
P_{x}^{\rho}\left(\sigma_{R} \leq t \mid Z(t)=\tilde{x}\right)=P_{\tilde{x}}^{\rho}\left(\sigma_{R} \leq t \mid Z(t)=x\right)=\frac{P_{\tilde{x}}^{\rho}\left(\sigma_{R} \leq t, Z(t)=x\right)}{P_{\tilde{x}}^{\rho}(Z(t)=x)} .
$$

The numerator equals

$$
P_{\tilde{x}}^{\rho}\left(\sigma_{R} \leq t, Z(t)=x\right)=E_{\tilde{x}}^{\rho}\left(\left.1\left\{\sigma_{R} \leq t\right\} P_{Z\left(\sigma_{R}\right)}^{\rho}(Z(t-s)=x)\right|_{s=\sigma_{R}}\right) .
$$

Since

$$
P_{z}^{\rho}(Z(t)=x) \geq P_{z}^{\rho}(Z(t-s)=x) P_{x}^{\rho}(Z(s)=x) \text { for all } z \in \mathbb{Z} \text { and } 0 \leq s \leq t,
$$

and since by ergodicity

$$
\inf _{s \geq 0} P_{x}^{\rho}(Z(s)=x)=c>0
$$

we obtain via (4.18) that

$$
P_{\tilde{x}}^{\rho}\left(\sigma_{R} \leq t \mid Z(t)=x\right) \leq \frac{1}{c} P_{\tilde{x}}^{\rho}\left(\sigma_{R} \leq t\right) \frac{P_{R+1}(Z(t)=x)+P_{-R-1}(Z(t)=x)}{P_{\tilde{x}}^{\rho}(Z(t)=x)} .
$$

The quotient in the r.h.s. of (4.21) tends to 1 uniformly in $\tilde{x} \in[-R, R]$ when $t \rightarrow \infty$ and

$$
R \log R=o(t),
$$

as can be seen from (4.13) (use that $u$ is unimodal and centered at 0 ). Hence, to prove the claim in (4.16) it remains to show that $P_{\tilde{x}}^{\rho}\left(\sigma_{R} \leq t\right)$ tends to zero uniformly in $\tilde{x} \in[-R, R]$. For this we shall want to let $R$ grow sufficiently fast with $t$, but it will turn out that (4.22) can still be met.

Let

$$
\eta_{z}=\inf \{s \geq 0: Z(s)=z\} .
$$

Then

$$
\begin{aligned}
P_{\tilde{x}}^{\rho}\left(\sigma_{R} \leq t\right) & \leq P_{\tilde{x}}^{\rho}\left(\eta_{R+1} \leq t\right)+P_{\tilde{x}}^{\rho}\left(\eta_{-R-1} \leq t\right) \\
& \leq P_{R}^{\rho}\left(\eta_{R+1} \leq t\right)+P_{-R}^{\rho}\left(\eta_{-R-1} \leq t\right) \quad(\tilde{x} \in[-R, R]) .
\end{aligned}
$$


We shall only give the argument for the first term in the r.h.s. of (4.24), the second term being similar.

For $0 \leq n \leq R$, define the event

$$
A_{R, n}=\text { the first } R-n \text { steps of the random walk go to the left. }
$$

Then we can estimate

$$
P_{R}^{\rho}\left(\eta_{R+1} \leq t\right)=P_{R}^{\rho}\left(\left[A_{R, n}\right]^{c}\right)+P_{R}^{\rho}\left(\eta_{R+1} \leq t, A_{R, n}\right) .
$$

We begin by looking at the first term in the r.h.s. of (4.26). Let $r(x)$ be the probability that a step from $x$ goes to the right. Then, by Theorem $2 \mathrm{I}(3)$ (iii),

$$
r(x)=\frac{v(x+1)}{v(x)}\left[\frac{v(x-1)}{v(x)}+\frac{v(x+1)}{v(x)}\right]^{-1} \sim \frac{1}{(2 \rho x \log x)^{2}} \quad(x \rightarrow \infty) .
$$

(Recall that $u$ and $v$ are linked as $u=v /\|v\|_{\ell^{2}}$; the $\rho$-dependence is suppressed from the notation.) Therefore for $n \rightarrow \infty$

$$
P_{R}^{\rho}\left(A_{R, n}\right)=\prod_{x=n+1}^{R}(1-r(x))=\exp \left[-\frac{1}{4 \rho^{2}}(1+o(1)) \sum_{x=n+1}^{R} \frac{1}{(x \log x)^{2}}\right]
$$

and it follows that

$$
\lim _{n \rightarrow \infty} \inf _{R \geq n} P_{R}^{\rho}\left(A_{R, n}\right)=1 .
$$

Thus we have proved that the first term in the r.h.s. of (4.26) tends to zero as $n \rightarrow \infty$ uniformly in $R \geq n$.

Let us now turn to the second term in the r.h.s. of (4.26). Because

$$
P_{R}^{\rho}\left(\eta_{R+1} \leq t \mid A_{R, n}\right) \leq P_{n}^{\rho}\left(\eta_{R+1} \leq t\right)
$$

we see from (4.29) that it suffices to show that the r.h.s. of (4.30) tends to zero. By Markov's inequality

$$
P_{n}^{\rho}\left(\eta_{R+1} \leq t\right) \leq \inf _{\gamma>0} e^{\gamma t} \prod_{x=n}^{R} E_{x}^{\rho}\left(e^{-\gamma \eta_{x+1}}\right)
$$

Next, starting from $x$ the time $\eta_{x+1}$ to reach $x+1$ is bounded from below by

$$
\eta_{x+1} \geq \sum_{k=1}^{\nu_{x}} \xi_{x, k}
$$

where $\xi_{x, k}$ is the sojourn time at $x$ prior to the $k$-th jump from $x$ and $\nu_{x}$ is the number of jumps from $x$ going to the left before hitting $x+1$. Now, the $\xi_{x, k}$ 's are i.i.d. exponentially 
distributed with mean given by the second factor in the r.h.s. of (4.27), while $\nu_{x}$ is geometrically distributed with mean $1 / r(x)$. Hence the r.h.s. of (4.32) is exponentially distributed with mean $v(x) / v(x+1)$. Therefore

$$
E_{x}^{\rho}\left(e^{-\gamma \eta_{x+1}}\right) \leq \frac{1}{1+\gamma \frac{v(x)}{v(x+1)}} \leq \frac{1}{\gamma} \frac{v(x+1)}{v(x)} .
$$

Substitute (4.33) into (4.31), pick $\gamma=1 / R$ and $n=\lfloor R / 2\rfloor$, and use that $v(x+1) / v(x) \sim$ $2 p x \log x(x \rightarrow \infty)$, to arrive at

$$
P_{\lfloor R / 2\rfloor}^{\rho}\left(\eta_{R+1} \leq t\right) \leq \exp \left[\frac{t}{R}-(1+o(1)) \frac{R}{2} \log \log R\right]
$$

where $o(1)$ holds for $R \rightarrow \infty$ uniformly in $t$. The r.h.s. tends to zero as $R \rightarrow \infty$ when

$$
t=o\left(R^{2} \log \log R\right)
$$

Combining (4.24), (4.26), (4.29-4.30) and (4.34), we have proved that the 1.h.s. of (4.24) tends to zero, provided (4.22) and (4.35) are met. The latter are exactly what determines the restrictions on $R$ and $t$ in (4.16).

\section{$5 \quad$ Functional analysis}

In this section we analyze the variational problem $(* *)$ of Section 0.4 and its relation to the nonlinear difference equation $(*)$ of Section 0.3. Proposition 3 will be proved in Section 5.1, Theorem 2 in Section 5.2. Section 5.3 contains Lemma 16 and its proof. This lemma was already used in Section 3.1. Throughout most of this section $\rho$ will be suppressed from the notation.

\subsection{Proof of Proposition 3}

Fix $\rho \in(0, \infty)$ and let $F_{d}: \mathcal{P}_{d} \rightarrow[0, \infty]$ be the functional

$$
F_{d}(p)=I_{d}(p)+\rho J_{d}(p)
$$

with $I_{d}, J_{d}$ defined in $(0.16-0.17)$ and $\mathcal{P}_{d}=\mathcal{P}\left(\mathbb{Z}^{d}\right)$. Then (**) reads

$$
\chi(\rho)=\frac{1}{2 d} \inf _{p \in \mathcal{P}_{d}} F_{d}(p) .
$$

$F_{d}$ is lower semicontinuous in the weak topology. $\mathcal{P}_{d}$ is not compact in the weak topology, but with an easy argument we shall be able to show existence of a minimum. However, the trouble with $(5.2)$ is that $F_{d}$ is the sum of a convex part, $I_{d}$, and a concave part, $\rho J_{d}$. Therefore uniqueness of the minimum is a more subtle problem. 


\subsubsection{Analysis of (**)}

Lemma $13(a) \inf _{p \in \mathcal{P}_{d}} F_{d}(p)=\operatorname{dinf}_{p \in \mathcal{P}_{1}} F_{1}(p)$.

(b) Let $\mathcal{M}_{d} \subseteq \mathcal{P}_{d}$ denote the set of minimizers of $F_{d}$. Then $\mathcal{M}_{1} \neq \emptyset$ and $\mathcal{M}_{d}=\left(\mathcal{M}_{1}\right)^{\otimes d}$.

(c) All $p \in \mathcal{M}_{1}$ are strictly positive.

Proof. (a) The proof is by induction on the dimension $d$. The claim is obviously true for $d=1$. Suppose that it holds for all dimensions $\leq d$. Pick any $p \in \mathcal{P}_{d+1}$. Let $p_{d} \in \mathcal{P}_{d}$ and $p_{1} \in \mathcal{P}_{1}$ be the marginals of $p$ on the coordinates numbered $1, \ldots, d$ resp. $d+1$, i.e.,

$$
\begin{array}{ll}
p_{d}(x)=\sum_{z \in \mathbb{Z}} p(x, z) & \left(x \in \mathbb{Z}^{d}\right) \\
p_{1}(z)=\sum_{x \in \mathbb{Z}^{d}} p(x, z) & (z \in \mathbb{Z}) .
\end{array}
$$

Define the conditional probability measures

$$
\begin{aligned}
& q_{1}(z \mid x)=p(x, z) / p_{d}(x) \\
& q_{d}(x \mid z)=p(x, z) / p_{1}(z) .
\end{aligned}
$$

(If $p_{d}(x)=0$ then set $q_{1}(z \mid x)=0$ for all $z$, etc.) One easily checks from $(0.16-0.17)$ that

$$
\begin{aligned}
I_{d+1}(p(\cdot))= & \sum_{x} p_{d}(x) I_{1}\left(q_{1}(\cdot \mid x)\right)+\sum_{z} p_{1}(z) I_{d}\left(q_{d}(\cdot \mid z)\right) \\
J_{d+1}(p(\cdot))= & \sum_{x} p_{d}(x) J_{1}\left(q_{1}(\cdot \mid x)\right)+\sum_{z} p_{1}(z) J_{d}\left(q_{d}(\cdot \mid z)\right) \\
& +\left\{\sum_{x} p_{d}(x)\left[\sum_{z} q_{1}(z \mid x) \log q_{1}(z \mid x)\right]-\sum_{z} p_{1}(z) \log p_{1}(z)\right\} .
\end{aligned}
$$

Because $q \rightarrow q \log q(q \geq 0)$ is strictly convex and $\sum_{x} p_{d}(x) q_{1}(z \mid x)=p_{1}(z)$, it follows from Jensen's inequality that the term between braces in (5.5) is $\geq 0$ with equality iff $q_{1}(z \mid x)$ is constant in $x$ for all $z$, i.e.,

$$
p=p_{d} \otimes p_{1} .
$$

By combining (5.1) and (5.5) we get

$$
F_{d+1}(p) \geq \sum_{x} p_{d}(x) F_{1}\left(q_{1}(\cdot \mid x)\right)+\sum_{z} p_{1}(z) F_{d}\left(q_{d}(\cdot \mid z)\right)
$$

Varying over $p$ we obtain

$$
\inf _{p \in \mathcal{P}_{d+1}} F_{d+1}(p) \geq \inf _{p \in \mathcal{P}_{1}} F_{1}(p)+\inf _{p \in \mathcal{P}_{d}} F_{d}(p) .
$$

Since $F_{d+1}(p)=F_{1}\left(p_{1}\right)+F_{d}\left(p_{d}\right)$ for all $p$ of the form (5.6), we have proved that the claim holds for dimension $d+1$ and therefore completed the induction step.

(b) The argument in (a) shows that $\mathcal{M}_{d}=\left(\mathcal{M}_{1}\right)^{\otimes d}$. We next prove that $\mathcal{M}_{1} \neq \emptyset$. For ease of notation we shall henceforth suppress the dimension index 1 . The proof comes in 2 Steps. 
Step 1: For every $p \in \mathcal{P}$ with $F(p)<\infty$ there exists a $\tilde{p} \in \mathcal{P}$ such that:

(i) $F(\hat{p}) \leq F(p)$, with strict inequality when $p$ is not unimodal.

(ii) $\tilde{p}$ is unimodal.

(iii) $\tilde{p}$ is a permutation of $p$ (i.e., $\tilde{p}(x)=p(\pi(x))$ for some permutation $\pi$ of $\mathbb{Z}$ ).

Proof. The proof is by induction. We shall show how to construct a sequence $\left(p_{n}\right)_{n \geq 1}$ in $\mathcal{P}$ satisfying $p_{1}=p$ and the following properties:

(i') $F\left(p_{n+1}\right) \leq F\left(p_{n}\right)(n \geq 1)$.

(i") For every $n \geq 1$ and $1 \leq m \leq n$ : the positions of the first $m$ 'record values' (i.e., largest values) of $p_{n}$ form a cluster.

(iii') For every $n \geq 1: p_{n+1}$ is a permutation of $p_{n}$ attaching the $(n+1)$-st record value of $p_{n}$ to the cluster consisting of the previous record values.

The construction goes as follows. Pick any sequence $\left(x_{n}\right)_{n \geq 1}$ along which the values of $p$ are arranged in decreasing order. Given $p_{n}$, let $\left[u_{n}, v_{n}\right]$ be the cluster consisting of the positions of the first $n$ record values. Without loss of generality we may assume that $x_{n+1}>v_{n}$ (the case $x_{n+1}<u_{n}$ being similar). If $x_{n+1}=v_{n}+1$ then put $p_{n+1}=p_{n}$. Otherwise put

$$
\begin{array}{rlrl}
p_{n+1}(y) & =p_{n}(y) & & \text { for } y \leq v_{n} \text { and } y>x_{n+1} \\
& =p_{n}\left(x_{n+1}\right) & & \text { for } y=v_{n}+1 \\
& =p_{n}(y+1) & \text { for } v_{n}<y<x_{n+1}
\end{array}
$$

i.e., attach the $(n+1)$-st record value to the cluster $\left[u_{n}, v_{n}\right]$ and close up the hole it leaves behind. It is clear from (5.9) that the sequence $\left(p_{n}\right)_{n>1}$ constructed in this way satisfies (ii') and (iii'). To prove (i') we recall that $F=I+\rho J$ with $I, J$ given by $(0.16-0.17)$. Now, $J\left(p_{n+1}\right)=J\left(p_{n}\right)(n \geq 1)$ because $J$ is invariant under permutations. Thus it suffices to show that $I\left(p_{n+1}\right) \leq I\left(p_{n}\right)(n \geq 1)$.

Recall that $I$ sums the square of the increments of $\sqrt{p}$ along the bonds of $\mathbb{Z}$. The only bonds where something changes in $(5.9)$ are $\left(v_{n}, v_{n}+1\right),\left(x_{n+1}-1, x_{n+1}\right)$ and $\left(x_{n+1}, x_{n+1}+1\right)$. Abbreviate

$$
\begin{aligned}
& a=\sqrt{p_{n}\left(v_{n}\right)}, b=\sqrt{p_{n}\left(x_{n+1}\right)}, c=\sqrt{p_{n}\left(v_{n}+1\right)}, \\
& d=\sqrt{p_{n}\left(x_{n+1}-1\right)}, e=\sqrt{p_{n}\left(x_{n+1}+1\right)} \\
& (a \geq b \geq c, d, e) .
\end{aligned}
$$

Then we easily compute

$$
\begin{aligned}
& I\left(p_{n}\right)-I\left(p_{n+1}\right) \\
& \quad=\left\{(a-c)^{2}+(d-b)^{2}+(b-e)^{2}\right\}-\left\{(a-b)^{2}+(b-c)^{2}+(d-e)^{2}\right\} \\
& \quad=2(a-b)(b-c)+2(b-d)(b-e) \geq 0 .
\end{aligned}
$$

Thus we have proved ( $\left.i^{\prime}\right)$. It is easily checked that if $p$ is not unimodal, then in (i') strict inequality holds for at least one $n \geq 1$ in the above iterative construction.

Finally, $\left(p_{n}\right)_{n \geq 1}$ is obviously pointwise convergent. The limit we call $\tilde{p}$, which obviously satisfies the claims because of ('iii') (recall that $F$ is lower semicontinuous). 
Step 2: $\inf F=\min F$.

Proof. Let $\left(q_{n}\right)$ be a minimizing sequence in $\mathcal{P}$, i.e., $\lim _{n \rightarrow \infty} F\left(q_{n}\right)=\inf _{p \in \mathcal{P}} F(p)$. Let $\tilde{q}_{n}$ be the permutation of $q_{n}$ obtained as in Step 1. Then also $\left(\tilde{q}_{n}\right)$ is a minimizing sequence. We shall prove that this sequence is tight modulo shifts. For ease of notation we drop the tilde.

Without loss of generality we may assume that the first record value of $q_{n}$ sits at $x=0$ for all $n$. Since $q_{n}$ has the cluster property (see Step 1(ii)), its first $m$ record values lie inside the interval $[-m, m]$. Since there can be at most $m$ record values larger than $1 / m$, it follows that

$$
\sup _{x \notin[-m, m]} q_{n}(x) \leq \frac{1}{m} \text { for all } n, m .
$$

Now, there exists some $K<\infty$ such that $F\left(q_{n}\right) \leq K$ for all $n$ sufficiently large. Therefore, since all summands of $F$ are nonnegative (recall $(0.16-0.17)$ ), we must have

$$
-\rho \sum_{x \notin[-m, m]} q_{n}(x) \log q_{n}(x) \leq K .
$$

But from (5.12) follows

$$
-\rho \sum_{x \notin[-m, m]} q_{n}(x) \log q_{n}(x) \geq \rho \log m \sum_{x \notin[-m, m]} q_{n}(x) .
$$

Combining (5.13-5.14) we obtain

$$
\sum_{x \notin[-m, m]} q_{n}(x) \leq \frac{K}{\rho \log m} .
$$

Since this bound is uniform in $n$, we have proved tightness.

Thus $q_{n}$ converges to some $q \in \mathcal{P}$ along some subsequence. Now note that $F(q) \leq$ $\lim _{n \rightarrow \infty} F\left(q_{n}\right)=\inf F$ because $F$ is lower semicontinuous and $\left(q_{n}\right)$ is a minimizing sequence. Hence $q$ is a minimizer.

(c) The proof is by contradiction. Suppose that $p \in \mathcal{P}$ is not strictly positive. Then there exists some $x_{0}$ such that $p\left(x_{0}\right)=0$ and $p\left(x_{0}+1\right)+p\left(x_{0}-1\right)>0$. For $\epsilon \in(0,1)$, define $p_{\epsilon} \in \mathcal{P}$ as

$$
p_{\epsilon}(x)= \begin{cases}(1-\epsilon) p(x) & x \neq x_{0} \\ \epsilon & x=x_{0} .\end{cases}
$$

One easily deduces from $(0.16-0.17)$ and (5.1) that

$$
\begin{gathered}
F\left(p_{\epsilon}\right)=(1-\epsilon) F(p)+2\left\{\epsilon-\sqrt{\epsilon(1-\epsilon)}\left[\sqrt{p\left(x_{0}-1\right)}+\sqrt{p\left(x_{0}+1\right)}\right]\right\} \\
-\rho\{\epsilon \log \epsilon+(1-\epsilon) \log (1-\epsilon)\} .
\end{gathered}
$$

As $\epsilon \rightarrow 0$, the term with $\sqrt{\epsilon(1-\epsilon)}$ is dominant. Hence $F\left(p_{\epsilon}\right)<F(p)$ for all $\epsilon$ sufficiently small, so $p$ is not a minimizer.

This completes the proof of Lemma 13. 


\subsubsection{The link between $(* *)$ and $(*)$}

Let

$$
\mathcal{V}=\left\{v: \mathbb{Z} \rightarrow \mathbb{R}^{+}: v \text { solves }(*)\right\} .
$$

Lemma 14 (a) $\mathcal{V} \neq \emptyset$ and $\inf _{v \in \mathcal{V}}\|v\|_{\ell^{2}}=\min _{v \in \mathcal{V}}\|v\|_{\ell^{2}}$.

(b) Let $\mathcal{V}$ be the set of minimizers in (a). Then

$$
\begin{aligned}
\mathcal{M} & =\left\{v^{2} /\|v\|_{\ell^{2}}^{2}: v \in \mathcal{V}\right\} \\
F\left(v^{2} /\|v\|_{\ell^{2}}^{2}\right) & =2 \rho \log \|v\|_{\ell^{2}} \quad(v \in \mathcal{V}) .
\end{aligned}
$$

Proof. (a-b) Let $p \in \mathcal{M}$ be any minimizer of $F$. Since $p$ is strictly positive by Lemma $13(\mathrm{c})$, we can do a standard variational argument. Indeed, pick any $h: \mathbb{Z} \rightarrow \mathbb{R}$ with finite support and $\sum_{z} h(z)=0$. Since $p+\epsilon h \in \mathcal{P}_{1}$ for $\epsilon$ small enough, we compute from $(0.16-0.17)$ and (5.1)

$$
\begin{aligned}
0 & \leq \lim _{\epsilon \rightarrow 0} \frac{1}{\epsilon}[F(p+\epsilon h)-F(p)] \\
& =\sum_{z}(\sqrt{p(z+1)}-\sqrt{p(z)})\left(\frac{h(z+1)}{\sqrt{p(z+1)}}-\frac{h(z)}{\sqrt{p(z)}}\right)-\rho \sum_{z} h(z)(1+\log p(z)) \\
& =\sum_{z} h(z)\left\{-\sqrt{\frac{p(z+1)}{p(z)}}-\sqrt{\frac{p(z-1)}{p(z)}}+2-\rho \log p(z)\right\} .
\end{aligned}
$$

Hence, $h$ being arbitrary, there exists a constant $\lambda$ such that

$$
\left\{-\sqrt{\frac{p(z+1)}{p(z)}}-\sqrt{\frac{p(z-1)}{p(z)}}+2-\rho \log p(z)\right\}=\lambda \quad(z \in \mathbb{Z}) .
$$

Put

$$
v(z)=e^{\lambda / 2 p} \sqrt{p(z)}
$$

Then (5.22) transforms into

$$
\frac{v(z+1)}{v(z)}+\frac{v(z-1)}{v(z)}-2+2 p \log v(z)=0
$$

which is $(*)$. Moreover, via (5.22-5.23) and the definition of $F$,

$$
\begin{aligned}
F(p) & =\sum_{z}(\sqrt{p(z+1)}-\sqrt{p(z)})^{2}-\rho \sum_{z} p(z) \log p(z) \\
& =\sum_{z} \lambda p(z)=\lambda=2 \rho \log \|v\|_{\ell^{2}}
\end{aligned}
$$

Thus, with each $p \in \mathcal{M}$ corresponds a solution of $(*)$ given by $v^{2}=p \exp (\min F / \rho)$ (or $\min F=2 \rho \log \|v\|_{\ell^{2}}$. Hence $\mathcal{M} \subseteq\left\{v^{2} /\|v\|_{\ell^{2}}^{2}: v \in \mathcal{V}\right\}$. Since we know from Lemma 13(b) that $\mathcal{M} \neq \emptyset$, this implies that $\mathcal{V} \neq \emptyset$.

Reversely, given any $v \in \mathcal{V}$, one easily checks that $p$ defined by $p=v^{2} /\|v\|_{\ell^{2}}^{2}$ satisfies $F(p)=2 \rho \log \|v\|_{\ell^{2}}$. Hence, only the solutions $v \in \mathcal{V}$ correspond to the minimizers $p \in$ $\mathcal{M}$.

Lemmas 13-14 prove Proposition 3. 


\subsection{Proof of Theorem 2}

\subsubsection{Parts $(1-2)$ and $(3)($ ii - iii $)$}

We already know from Lemmas 13-14 that (*) has a ground state, so Part (1) is covered. Part (2) is immediate from Lemma $14(\mathrm{~b})$ and the fact that $v^{2} /\|v\|_{\ell^{2}}^{2}$ satisfies the tightness property of (5.15).

Lemma 15 Any $v \in \mathcal{V}$ satisfies:

(a) $1 \leq\|v\|_{\ell^{2}} \leq \exp (1 / \rho)$.

(b) If $v(x) \geq v(y)$ for $y=x+1, x-1$ with at most equality at one point, then $v(x)>1$. Similarly with both inequalities reversed.

Proof. (a) By Lemma 14(b)

$$
2 \rho \log \|v\|_{\ell^{2}}=\inf _{p \in \mathcal{P}_{1}} F(p) .
$$

The lower bound follows because $F \geq 0$. The upper bound follows by picking the trial function $p=\delta_{0}$, for which $F\left(\delta_{0}\right)=I\left(\delta_{0}\right)+\rho J\left(\delta_{0}\right)=2+\rho \cdot 0=2$.

(b) If $x$ is a local maximum of $v$ then $\Delta v(x)<0$. Hence $2 \rho v(x) \log v(x)>0$. Similarly for a local minimum.

We know from Step 1 in the proof of Lemma 13 that $v$ has the cluster property, i.e., $v$ is unimodal. A maximum of three or more points is not possible, since (**) would give that $v \equiv C>0$, which is not in $l^{2}(\mathbb{Z})$. Thus we have proved Part (3)(i). Part (3)(ii) now follows from Lemma 15(b). Indeed, if there were an $x$ such that $v(x)<v(x+1)=$ $v(x+2)<v(x+3)$, then this would contradict $v(x+1)>1>v(x+2)$. Similarly with the inequalities reversed.

\subsubsection{Parts (4) and (5)}

We shall prove Part (4) for large $\rho$ by contradiction. Suppose that $(*)$ has two ground states, $v_{1}, v_{2} \in \mathcal{V}$, which are not translates of each other. By shifting them we can always arrange that $v_{i}(0)=\max _{x} v_{i}(x)>1(i=1,2)$. Without loss of generality we may assume $v_{1}(0) \geq v_{2}(0)$.

Define $w$ and $v_{1,2}$ by

$$
\begin{array}{ll}
w & =v_{1}-v_{2} \\
1+\log v_{1,2} & =\frac{v_{1} \log v_{1}-v_{2} \log v_{2}}{v_{1}-v_{2}} .
\end{array}
$$

Since $v_{1}, v_{2}$ both solve $(*)$, we have

$$
\Delta w+2 \rho w\left(1+\log v_{1,2}\right)=0 .
$$

Next note the following properties:

(i') $v_{1,2}$ lies everywhere inbetween $v_{1}$ and $v_{2}$. 
(ii') $v_{1,2}(0)>1$.

(iii') if $\rho \geq 2 / \log \left(1+e^{-2}\right)$ then $v_{1,2}(x)<e^{-1}$ for all $x \neq 0$.

Indeed, (i') follows from the mean value theorem, (ii') follows from (i') and $v_{i}(0)>1(i=$ 1,2), while (iii') follows from (i'-ii') and Lemma 15(a) giving $\sum_{x \neq 0} v_{i}^{2}(x) \leq \exp (2 / \rho)-v_{i}^{2}(0)$ $<\exp (2 / \rho)-1 \leq 1 / e^{2}(i=1,2)$.

Now argue as follows. From (iii') together with (5.28) we get

$$
w(x) \text { and } \Delta w(x) \text { have the same sign for all } x \neq 0 \text {. }
$$

At $x=0$, on the other hand, (5.28) can be written as

$$
w(1)+w(-1)=2 w(0)\left\{1-\rho\left(1+\log v_{1,2}(0)\right)\right\} .
$$

Suppose that $w(0)>0$ (the case $w(0)=0$ will be handled later). Then (5.30) and (ii') imply $w(1)+w(-1)<0$ (note that $\rho \geq 2 / \log \left(1+e^{-2}\right)>1$ ). Without loss of generality we may assume $w(1)<0$. Writing $\nabla w(x)=w(x)-w(x+1)$ and using (5.29), we deduce

$$
\left\{\begin{array} { l } 
{ \nabla w ( 0 ) > 0 } \\
{ w ( 1 ) < 0 }
\end{array} \Longrightarrow \left\{\begin{array}{l}
\nabla w(1)>\nabla w(0)>0 \\
w(2)<w(1)<0
\end{array}\right.\right.
$$

This implication can be iterated to yield that $x \rightarrow \nabla w(x)$ is strictly increasing for all $x \geq 0$. This in turn implies that $w(x)<w(0)-x \nabla w(0)(x \geq 2)$ and hence $\lim _{x \rightarrow \infty} w(x)=-\infty$. But now we have a contradiction because $v_{1}, v_{2} \in l^{2}(\mathbb{Z})$.

Finally, if $w(0)=0$ then (5.30) gives $w(1)+w(-1)=0$. It is not possible that $w(1)=w(-1)=0$. Namely, this would imply $v_{1}(x)=v_{2}(x)$ for $x=1,0,-1$ and hence $v_{1} \equiv v_{2}$ because $(*)$ is second order. Again, without loss of generality we may assume $w(1)<0$, and the argument proceeds as before. This completes the proof of Part (4).

If $v$ solves $(*)$ then so does $-v$. Hence the uniqueness of the ground state, proved above, implies that $v$ is symmetric about its maximum at 0 . This completes the proof of Part (5).

\subsubsection{Part (3)(iii)}

Define $r(x)=v(x) / v(x+1)$. This ratio satisfies the equation

$$
\frac{1}{r(x)}-2+r(x-1)=-2 \rho \log v(x),
$$

which can be rewritten in the forward form

$$
\frac{1}{r(x)}=K-r(x-1)+2 \rho \log \left[\prod_{y=0}^{x-1} r(y)\right] \quad(x \geq 1)
$$

with $K=2-2 \rho \log v_{\rho}(0)$. The unimodality of $v_{\rho}$ (Part (3)(ii)) implies that $r(x) \geq 1(x \geq$ $0)$. It therefore follows from (5.33) that

$$
(K-1)+2 \rho \sum_{y=0}^{x-1} \log r(y) \leq r(x-1) \leq K+2 \rho \sum_{y=0}^{x-1} \log r(y) .
$$


By combining upper and lower bound we get

$$
-1+2 \rho \log r(x) \leq r(x)-r(x-1) \leq 1+2 \rho \log r(x) .
$$

We must show that (5.35) implies $r(x) \sim 2 \rho x \log x(x \rightarrow \infty)$, as claimed. We shall do this via a comparison with the continuous equation $f^{\prime}=2 \rho \log f$.

Lower bound: Let $f:\left[x_{0}, \infty\right) \rightarrow \mathbb{R}^{+}$be the solution of the differential equation

$$
\begin{aligned}
& f^{\prime}=-1+2 \rho \log f \\
& f\left(x_{0}\right)=r\left(x_{0}\right),
\end{aligned}
$$

where the starting point $x_{0}$ is to be chosen large enough so that $r\left(x_{0}\right) \geq 2 \rho \vee \exp (1 / 2 \rho)$. Note that such an $x_{0}$ always exists because $\lim _{x \rightarrow \infty} r(x)=\infty$ (as is easily seen from (5.32) using that $\lim _{x \rightarrow \infty} v(x)=0$ and $\left.r(x) \geq 1\right)$. We shall first show that $r(x) \geq f(x)$ for all $x \geq x_{0}$ and then that $f(x) \sim 2 \rho x \log x(x \rightarrow \infty)$.

Since $f\left(x_{0}\right) \geq \exp (1 / 2 \rho)$, it follows from (5.36) that $f$ is increasing. Hence

$$
\begin{aligned}
f(x)-f(x-1) & =\int_{x-1}^{x} d y f^{\prime}(y) \\
& =\int_{x-1}^{x} d y[-1+2 \rho \log f(y)] \\
& \leq-1+2 \rho \log f(x) \quad\left(x \geq x_{0}+1\right) .
\end{aligned}
$$

Define $g: \mathbb{R}^{+} \rightarrow \mathbb{R}$ by $g(u)=u-(-1+2 \rho \log u)$. Then (5.37) can be rewritten as $f(x-1) \geq g(f(x))$. From the lower bound in (5.35), on the other hand, we know that $r(x-1) \leq g(r(x))$. Therefore we obtain

$$
\begin{aligned}
& g^{-1}(f(x-1)) \geq f(x) \\
& g^{-1}(r(x-1)) \leq r(x) \quad\left(x \geq x_{0}+1\right) .
\end{aligned}
$$

Here we have used that $g, g^{-1}$ are strictly increasing on $[2 \rho, \infty)$ and that $f, r \geq 2 \rho$ on $\left[x_{0}, \infty\right)$. The latter holds because $f\left(x_{0}\right)=r\left(x_{0}\right) \geq 2 \rho$ and because $f, r$ are both increasing (for $r$ this follows easily from the lower bound in (5.35) because $r\left(x_{0}\right) \geq \exp (1 / 2 \rho)$ ). From (5.38) we get the implication: $r(x-1) \geq f(x-1) \Longrightarrow r(x) \geq f(x)$, which proves $r(x) \geq f(x)\left(x \geq x_{0}\right)$.

Define $h: \mathbb{R}^{+} \rightarrow \mathbb{R}$ by $h(u)=\int^{u} d v /(-1+2 \rho \log v)$. Then (5.36) gives $h^{\prime}(f) f^{\prime} \equiv 1$. Hence

$$
h(f(x))-h\left(f\left(x_{0}\right)\right)=x-x_{0} \quad\left(x \geq x_{0}\right) .
$$

Since $h(u) \sim u / 2 \rho \log u(u \rightarrow \infty)$, it follows that $f(x) / 2 \rho \log f(x) \sim x(x \rightarrow \infty)$, which is the same as $f(x) \sim 2 \rho x \log x(x \rightarrow \infty)$.

Upper bound: By a similar argument. First rewrite the upper bound in (5.35) as $r(x-1) \geq$ $\overline{\bar{g}}(r(x))$ with $\bar{g}(u)=u-(1+2 \rho \log u)$. Next, define $\bar{f}$ to be the solution of the differential equation

$$
\begin{aligned}
& f^{\prime}=\bar{g}^{-1}(f)-f \\
& f\left(x_{0}\right)=r\left(x_{0}\right) .
\end{aligned}
$$


Since $u \rightarrow \bar{g}^{-1}(u)-u$ is asymptotically increasing and positive, we have

$$
\begin{aligned}
\bar{f}(x)-\bar{f}(x-1) & =\int_{x-1}^{x} d y \bar{f}^{\prime}(y) \\
& =\int_{x-1}^{x} d y\left[\bar{g}^{-1}(\bar{f}(y))-\bar{f}(y)\right] \\
& \geq \bar{g}^{-1}(\bar{f}(x-1))-\bar{f}(x-1) \quad\left(x \geq x_{0}+1\right),
\end{aligned}
$$

provided $x_{0}$ is again chosen large enough so that $r\left(x_{0}\right)$ falls in the asymptotic regime. Thus, we get precisely the reverse of (5.38), namely

$$
\begin{aligned}
& \bar{g}^{-1}(\bar{f}(x-1)) \leq \bar{f}(x) \\
& \bar{g}^{-1}(r(x-1)) \geq r(x) .
\end{aligned}
$$

Hence $r(x) \leq f(x)\left(x \geq x_{0}\right)$. Finally, let $\bar{h}=\int^{u} d v /\left(\bar{g}^{-1}(v)-v\right)$. Then again $\bar{h}^{\prime}(\bar{f}) \bar{f}^{\prime} \equiv 1$. Since $\bar{g}^{-1}(v)-v \sim 2 \rho \log v(v \rightarrow \infty)$, we again find $\bar{f}(x) \sim 2 \rho x \log x(x \rightarrow \infty)$.

\subsubsection{Parts (6) and (7)}

Part (6) is immediate from Lemma 15.

We shall henceforth write $F_{\rho}, p_{\rho}, v_{\rho}$ instead of $F, p, v$ in order to display the $\rho$-dependence. According to Lemma 14(b), the minimizers $p_{\rho}$ of $F_{\rho}$ and the ground states $v_{\rho}$ of $(*)$ are related as $p_{\rho}=v_{\rho}^{2} /\left\|v_{\rho}\right\|_{\ell^{2}}^{2}$ and $v_{\rho}^{2}=p_{\rho} \exp \left[F_{\rho}\left(p_{\rho}\right) / \rho\right]$. The behavior of these quantities as $\rho \rightarrow 0$ comes in 3 steps. The argument below is valid for any minimizer $p_{\rho}$ resp. ground state $v_{\rho}$ assumed to be centered at 0 .

Define

$$
\hat{p}_{\rho}(x)=\frac{1}{\sqrt{\rho}} p_{\rho}(\lfloor x / \sqrt{\rho}\rfloor) \quad(x \in \mathbb{R}),
$$

which is an element of $\mathcal{P}(\mathbb{R})$, the set of probability measures on $\mathbb{R}$.

Step 1: The family $\left(\hat{p}_{\rho}\right)_{\rho \in(0,1)}$ is:

(i) equicontinuous on compacts;

(ii) uniformly integrable;

(iii) uniformly bounded from above.

Proof. (i) Let $\hat{F}_{\rho}: \mathcal{P}(\mathbb{R}) \rightarrow[0, \infty)$ be the functional defined by (compare with $F_{\rho}$ defined in $(5.1))$

$$
\begin{aligned}
& \hat{F}_{\rho}=\hat{I}_{\rho}+\hat{J} \\
& \hat{I}_{\rho}(p)=\int_{\mathbb{R}} d x\left(\frac{1}{\sqrt{\rho}}[\sqrt{p(x+\sqrt{\rho})}-\sqrt{p(x)}]\right)^{2} \\
& \hat{J}(p)=-\int_{\mathbb{R}} d x p(x) \log p(x) .
\end{aligned}
$$


(Note that $\hat{J} \geq 0$ by Jensen's inequality, even though the integrand in $\hat{J}$ is not negative everywhere.) Then we have the relation (recall $(0.16-0.17)$ )

$$
\hat{F}_{\rho}\left(\hat{p}_{\rho}\right)=\frac{1}{\rho}\left[F_{\rho}\left(p_{\rho}\right)+\rho \log \sqrt{\rho}\right] .
$$

Because $p_{\rho}$ is a minimizer of $F_{\rho}$, we have $F_{\rho}\left(p_{\rho}\right) \leq F_{\rho}\left(q_{\rho}\right)$ for any trial function $q_{\rho} \in \mathcal{P}(\mathbb{Z})$. Pick $q_{\rho}(x)=(1-c) c^{|x|} /(1+c)(x \in \mathbb{Z})$ with $c=1-\sqrt{\rho}$. Then an easy computation gives $F_{\rho}\left(q_{\rho}\right)=-\rho \log \sqrt{\rho}+\left(\frac{3}{2}+\log 2\right) \rho+O\left(\rho^{3 / 2}\right)(\rho \rightarrow 0)$. Hence we conclude, using (5.45), that there exists some $K<\infty$ such that

$$
0 \leq \hat{F}_{\rho}\left(\hat{p}_{\rho}\right) \leq K \quad \text { for all } \rho \in(0,1)
$$

This, in turn, yields that for any $x, y \in \sqrt{\rho} \mathbb{Z}$ with $x>y$

$$
\begin{aligned}
\frac{K}{x-y} & \geq \frac{1}{x-y} \int_{y}^{x} d z\left(\frac{1}{\sqrt{\rho}}\left[\sqrt{\hat{p}_{\rho}(z+\sqrt{\rho})}-\sqrt{\hat{p}_{\rho}(z)}\right]\right)^{2} \\
& \geq\left(\frac{1}{x-y} \int_{y}^{x} d z \frac{1}{\sqrt{\rho}}\left[\sqrt{\hat{p}_{\rho}(z+\sqrt{\rho})}-\sqrt{\hat{p}_{\rho}(z)}\right]\right)^{2} \\
& =\left(\frac{1}{x-y}\left[\sqrt{\hat{p}_{\rho}(x)}-\sqrt{\hat{p}_{\rho}(y)}\right]\right)^{2} .
\end{aligned}
$$

The first inequality follows from (5.44) and (5.46), the second inequality from CauchySchwarz, the third equality from the fact that $\hat{p}_{\rho}$ is constant between the points of $\sqrt{\rho} \mathbb{Z}$. The estimate in (5.47) says that

$$
\left|\sqrt{\hat{p}_{\rho}(x)}-\sqrt{\hat{p}_{\rho}(y)}\right| \leq \sqrt{K|x-y|} \text { for all } x, y \in \sqrt{\rho} \mathbb{Z}
$$

which proves the claim.

(ii) By Jensen's inequality,

$$
-\int_{|x| \leq R} d x \hat{p}_{\rho}(x) \log \hat{p}_{\rho}(x) \leq-\left(\int_{|x| \leq R} d x \hat{p}_{\rho}(x)\right) \log \left(\int_{|x| \leq R} d x \hat{p}_{\rho}(x)\right) \leq \frac{1}{e} .
$$

Since $\hat{I}_{\rho}, \hat{J} \geq 0$, it now follows from (5.44) and (5.46) that

$$
-\int_{|x|>R} d x \hat{p}_{\rho}(x) \log \hat{p}_{\rho}(x) \leq K+\frac{1}{e} .
$$

Next, $p_{\rho}$ being unimodal, we have the same bound as in (5.12), namely $\sup _{|x|>m} p_{\rho}(x) \leq$ $1 / m$ for all $m \in \mathbb{N}, \rho>0$. In terms of $\hat{p}_{\rho}$ this bound translates into (pick $m=R / \sqrt{\rho}$ )

$$
\sup _{|x|>R} \hat{p}_{\rho}(x) \leq \frac{1}{R} \quad \text { for all } R \in \sqrt{\rho} \mathbf{N}, \rho>0 .
$$

Combining (5.50-5.51) we get

$$
\int_{|x|>R} d x \hat{p}_{\rho}(x) \leq\left(K+\frac{1}{e}\right) \frac{1}{\log R} \text { for all } R \in \sqrt{\rho} \mathbb{N}
$$


proving the claim.

(iii) Since $\int_{\mathbb{R}} d x \hat{p}_{\rho}(x)=1$ for all $\rho$, it immediately follows from (i) that $\hat{p}_{\rho}$ is bounded from above uniformly in $\rho \in(0,1)$.

Define

$$
\hat{v}_{\rho}(x)=v_{\rho}(\lfloor x / \sqrt{\rho}\rfloor) \quad(x \in \mathbb{R}),
$$

which is an element of $L^{2}(\mathbb{R})$.

Step 2: The family $\left(\hat{v}_{\rho}\right)_{\rho \in(0,1)}$ is:

(i) equicontinuous on compacts;

(ii) uniformly square integrable;

(iii) uniformly bounded from above;

(iv) uniformly bounded from below on compacts.

Proof. (i-iii) By (5.19), (5.43) and (5.45) we have the relation

$$
\hat{v}_{\rho}^{2}(x)=\hat{p}_{\rho} \exp \left[\hat{F}\left(\hat{p}_{\rho}\right)\right] .
$$

Therefore the claims follow from Step 1 via (5.46).

(iv) The proof of the uniform lower bound on compacts is more subtle and requires some work. We shall prove the claim on $\mathbb{R}_{+}$. The proof for $\mathbb{R}_{-}$is similar.

Pick $\rho \in(0,1)$ and let $v_{\rho}$ be any centered solution of $(*)$. We have

$$
\begin{aligned}
& \left(i^{\prime}\right)\left(\Delta v_{\rho}\right)(x)+2 \rho v_{\rho}(x) \log v_{\rho}(x)=0 \quad(x \in \mathbb{Z}) \\
& \left(i i^{\prime}\right) v_{\rho}(0)=\max _{x \in \mathbb{Z}} v_{\rho}(x)>1 \\
& \left(i i i^{\prime}\right) v_{\rho} \text { decreasing on } \mathbb{Z}_{+} .
\end{aligned}
$$

Multiply $(5.55)\left(\mathrm{i}^{\prime}\right)$ by $v_{\rho}(x-1)-v_{\rho}(x+1)$ to obtain

$$
\begin{aligned}
0= & {\left[v_{\rho}(x-1)-v_{\rho}(x+1)\right]\left[\left(\Delta v_{\rho}\right)(x)+2 \rho v_{\rho}(x) \log v_{\rho}(x)\right] } \\
= & {\left[v_{\rho}(x-1)-v_{\rho}(x)\right]^{2}-\left[v_{\rho}(x)-v_{\rho}(x+1)\right]^{2} } \\
& +2 \rho\left[v_{\rho}(x-1) v_{\rho}(x) \log v_{\rho}(x)-v_{\rho}(x+1) v_{\rho}(x) \log v_{\rho}(x)\right] .
\end{aligned}
$$

Define $x_{\rho} \in \mathbb{Z}_{+}$to be the unique point where

$$
v_{\rho}\left(x_{\rho}-1\right) \geq \frac{1}{e}>v_{\rho}\left(x_{\rho}\right) .
$$

Then, because $v \rightarrow v \log v$ is decreasing on the interval $[0,1 / e]$, it follows from (5.55)(iii') that

$$
\begin{aligned}
& v_{\rho}(x) \log v_{\rho}(x) \geq v_{\rho}(x-1) \log v_{\rho}(x-1) \\
& v_{\rho}(x) \log v_{\rho}(x) \leq v_{\rho}(x+1) \log v_{\rho}(x+1) .
\end{aligned}
$$


Substitution into $(5.56)$ gives

$$
\begin{aligned}
0 \geq & {\left[v_{\rho}(x-1)-v_{\rho}(x)\right]^{2}-\left[v_{\rho}(x)-v_{\rho}(x+1)\right]^{2} } \\
& +2 \rho\left[v_{\rho}^{2}(x-1) \log v_{\rho}(x-1)-v_{\rho}^{2}(x+1) \log v_{\rho}(x+1)\right] \quad\left(x \geq x_{\rho}+1\right) .
\end{aligned}
$$

Next, pick $y \geq x_{\rho}+1$ and $\operatorname{sum}(5.59)$ over all $x \geq y$. Then we get

$$
\begin{aligned}
0 \geq & {\left[v_{\rho}(y-1)-v_{\rho}(y)\right]^{2} } \\
& +2 \rho\left[v_{\rho}^{2}(y-1) \log v_{\rho}(y-1)+v_{\rho}^{2}(y) \log v_{\rho}(y)\right] \quad\left(y \geq x_{\rho}+1\right),
\end{aligned}
$$

where we use that $\lim _{x \rightarrow \infty} v_{\rho}(x)=0$. Bring the 2 under the logarithm and use once more the monotonicity, to obtain

$$
0 \geq\left[v_{\rho}(y-1)-v_{\rho}(y)\right]^{2}+2 \rho v_{\rho}^{2}(y-1) \log v_{\rho}^{2}(y-1) .
$$

Putting $y=x+1$ we thus arrive at

$$
v_{\rho}(x+1) \geq v_{\rho}(x)\left[1-2 \sqrt{\rho \log \left(\frac{1}{v_{\rho}(x)}\right)}\right] \quad\left(x \geq x_{\rho}\right) .
$$

This is a forward iterative inequality.

We shall iterate (5.62) until $v_{\rho}(x)$ drops below a threshold $\delta>0$. Later we shall see how to manipulate $\delta$. As part of the argument we shall need the following property:

$$
\inf _{\rho \in(0,1)} v_{\rho}\left(x_{\rho}\right)=\epsilon>0
$$

The proof of (5.63) comes at the end.

As long as $v_{\rho}(x) \geq \delta$, the term between square brackets in (5.62) is bounded from below by $1-2 \sqrt{\rho \log (1 / \delta)}$. Hence, by $(5.63)$,

$$
v_{\rho}(x) \geq \epsilon\left[1-2 \sqrt{\rho \log \left(\frac{1}{\delta}\right)}\right]^{x-x_{\rho}}
$$

for all $x \geq x_{\rho}$ such that the r.h.s. is $\geq \delta$. Now, since $1-u \geq e^{-2 u}$ for $u \in[0,1 / 2]$, we obtain (recall (5.55)(iii') and (5.63))

$$
v_{\rho}(x) \geq \epsilon \exp \left[-4 \sqrt{\rho \log \left(\frac{1}{\delta}\right)} x\right]
$$

for all $x \geq x_{\rho}$ such that the r.h.s. is $\geq \delta$, provided

$$
2 \sqrt{\rho \log \left(\frac{1}{\delta}\right)} \leq \frac{1}{2}
$$

Next, (5.65) is trivially true for $0 \leq x<x_{\rho}$ by (5.55)(iii'), (5.57) and (5.63). Therefore we can now scale $x$ to $\lfloor x / \sqrt{\rho}\rfloor$ to arrive at the lower bound

$$
v_{\rho}(\lfloor x / \sqrt{\rho}\rfloor) \geq \epsilon \exp \left[-4 \sqrt{\log \left(\frac{1}{\delta}\right)} x\right]
$$


for $x \geq 0$, still subject to (5.66) and to the requirement that the r.h.s. be $\geq \delta$. Now, for any $\delta>0$ the r.h.s. of (5.67) is bounded from below on compacts. Thus, all we have to check is that it is $\geq \delta$ on an interval $[0, x(\delta)]$ with $\lim _{\delta \rightarrow 0} x(\delta)=\infty$. But we in fact have $x(\delta)=\log (\epsilon / \delta) / 4 \sqrt{\log (1 / \delta)}$ and so this is indeed the case (irrespective of $\epsilon>0$ ). The condition (5.66) holds for any $\delta>0$ when $\rho$ is sufficiently small, so our proof of the uniform lower bound on compacts is complete.

It remains to check (5.63). For this we shall need the following fact, which follows from Step 2(iii) already proved:

$$
\sup _{\rho \in(0,1)} v_{\rho}(0)=c<\infty .
$$

It follows from (5.55)(ii'-iii') and (5.68) that $2 v_{\rho}(x) \log v_{\rho}(x) \leq C$ uniformly in $x$ with $C=2 c \log c$. Hence

$$
v_{\rho}(x-1)-v_{\rho}(x) \geq v_{\rho}(x)-v_{\rho}(x+1)-\rho C .
$$

Iteration from $x=0$ to $x=x_{\rho}$ gives (recall (5.55)(ii'))

$$
0 \geq v_{\rho}(-1)-v_{\rho}(0) \geq v_{\rho}\left(x_{\rho}-1\right)-v_{\rho}\left(x_{\rho}\right)-x_{\rho} \rho C
$$

or

$$
x_{\rho} \geq \frac{1}{\rho C}\left[v_{\rho}\left(x_{\rho}-1\right)-v_{\rho}\left(x_{\rho}\right)\right] .
$$

Now, suppose that there exists a $\delta>0$ and a sequence $\left(\rho_{k}\right)$ tending to zero such that

$$
v_{\rho_{k}}\left(x_{\rho_{k}}-1\right)-v_{\rho_{k}}\left(x_{\rho_{k}}\right) \geq \delta \text { for all } k \text {. }
$$

Then $x_{\rho_{k}} \geq\left\lfloor\delta / \rho_{k} C\right\rfloor$. It follows from (5.72) and iteration of (5.69) that

$$
v_{\rho_{k}}(x-1)-v_{\rho_{k}}(x) \geq \delta-\left(x_{\rho_{k}}-x\right) \rho_{k} C \quad\left(x \leq x_{\rho_{k}}\right)
$$

and hence

$$
v_{\rho_{k}}\left(x_{\rho_{k}}-l-1\right) \geq v_{\rho_{k}}\left(x_{\rho_{k}}-1\right)+\sum_{m=1}^{l}\left(\delta-m \rho_{k} C\right) \quad(l \geq 0) .
$$

Using (5.55)(ii') and picking $l=\left\lfloor\delta / \rho_{k} C\right\rfloor-1$, we arrive at

$$
v_{\rho_{k}}(0) \geq v_{\rho_{k}}\left(x_{\rho_{k}}-\left\lfloor\frac{\delta}{\rho_{k} C}\right\rfloor\right) \geq \frac{\delta}{2}\left(\left\lfloor\frac{\delta}{\rho_{k} C}\right\rfloor-1\right) .
$$

But clearly this contradicts (5.68). Hence (5.72) must fail and so we conclude that

$$
\lim _{\rho \rightarrow 0}\left[v_{\rho}\left(x_{\rho}-1\right)-v_{\rho}\left(x_{\rho}\right)\right]=0 \text {. }
$$

But, because of (5.57), this implies that

$$
\lim _{\rho \rightarrow 0} v_{\rho}\left(x_{\rho}\right)=\frac{1}{e}>0,
$$

which in turn implies (5.63). 


\section{Define}

$$
\hat{\mathcal{V}}=\left\{\hat{v} \in L^{2}(\mathbb{R}): \hat{v} \text { is a weak limit point of } \hat{v}_{\rho} \text { as } \rho \rightarrow 0\right\} .
$$

Step 3: (i) $\hat{\mathcal{V}} \neq \emptyset$.

(ii) For each $\hat{v} \in \hat{\mathcal{V}}$ the convergence is uniform on compacts in $\mathbb{R}$.

(iii) All $\hat{v} \in \hat{\mathcal{V}}$ are solutions of the differential equation $\hat{v}^{\prime \prime}+2 \hat{v} \log \hat{v}=0$.

Proof. (i) Step 2(i) implies that $\left(\hat{v}_{\rho}\right)_{\rho \in(0,1)}$ is relatively compact (in the set of continuous functions on $[-R, R]$ for arbitrary $R>0$ ).

(ii) Arzela-Ascoli.

(iii) Substitution of (5.53) into $(*)$ shows that $\hat{v}_{p}$ satisfies the equation

$$
\Delta_{\sqrt{\rho}} \hat{v}_{\rho}+2 \hat{v}_{\rho} \log \hat{v}_{\rho}=0
$$

with $\Delta_{\sqrt{\rho}}$ defined by

$$
\left(\Delta_{\sqrt{\rho}} f\right)(x)=\frac{1}{\rho}[f(x+\sqrt{\rho})-2 f(x)+f(x-\sqrt{\rho})] \quad(x \in \mathbb{R}) .
$$

Now, $\Delta_{\sqrt{\rho}}$ is the generator of simple random walk on $\sqrt{\rho} \mathbb{Z}$ with jump rate $2 / \rho$. Let us write $\hat{Z}_{\rho}=\left\{\hat{Z}_{\rho}(t): t \geq 0\right\}$ to denote this process and $P_{x}^{\Delta \sqrt{\rho}}, E_{x}^{\Delta} \sqrt{\rho}$ to denote its probability law and expectation. Then, using the Feynman-Kac formula, we have the representation

$$
\begin{gathered}
\hat{v}_{\rho}(x)=E_{x}^{\Delta \sqrt{\rho}}\left(\exp \left[\int_{0}^{\tau_{\rho}, R} d t \log \hat{v}_{\rho}\left(\hat{Z}_{\rho}(t)\right)\right] \hat{v}_{\rho}\left(\hat{Z}_{\rho}\left(\tau_{\rho, R}\right)\right)\right) \\
(x \in \sqrt{\rho} \mathbb{Z}, R \in \sqrt{\rho} \mathbb{N},|x|<R),
\end{gathered}
$$

where

$$
\tau_{\rho, R}=\inf \left\{t \geq 0:\left|\hat{Z}_{\rho}(t)\right|=R\right\} \quad(R \in \sqrt{\rho} \mathbb{Z}) .
$$

Next, let $B=\{B(t): t \geq 0\}$ be standard Brownian motion on $\mathbb{R}$, which is the Markov process with generator $\Delta$ (the Laplacian on $\mathbb{R}$ ). Then it is well known that there exists a coupling of $\left(\hat{Z}_{\rho}\right)_{\rho \in(0,1)}$ and $B$ such that

$$
\lim _{\rho \rightarrow 0} \sup _{t \in[0, T]}\left|\hat{Z}_{\rho}(t)-B(t)\right|=0 \quad \text { in probability for any } T>0 .
$$

Combining (5.81-5.83) with Step 3 we find that any $\hat{v} \in \hat{\mathcal{V}}$ must satisfy

$$
\hat{v}(x)=E_{x}^{\Delta}\left(\exp \left[\int_{0}^{\tau_{R}} d t \log \hat{v}(B(t))\right] \hat{v}\left(B\left(\tau_{R}\right)\right)\right) \quad\left(x \in \mathbb{R}, R \in \mathbb{R}^{+},|x|<R\right),
$$

where

$$
\tau_{R}=\inf \{t \geq 0:|B(t)|=R\} \quad(R \in \mathbb{R}) .
$$

To derive (5.84) from (5.83) we have used the following facts: 
(i') $\hat{v}_{\rho} \rightarrow \hat{v}$ uniformly on $[-R, R]$ as $\rho \rightarrow 0$ (by Step 3(ii)).

(ii') $x \rightarrow 2 \log \hat{v}_{\rho}(x)$ is bounded away from 0 and $\infty$ on $[-R, R]$ uniformly in $\rho \in(0,1)$ (by Step 2(iii-iv)).

(iii') $\left|\tau_{\rho, R}-\tau_{R}\right| \rightarrow 0$ as $\rho \rightarrow 0$ in probability (by (5.82),(5.83),(5.85)).

But (5.84) is the Feynman-Kac representation for the solution of $\hat{v}^{\prime \prime}+2 \hat{v} \log \hat{v}=0$.

To conclude the proof of Part (7), all that we need to do is recall footnote 4, which says that the solution of the limiting equation in Step 3(iii) is unique (modulo shifts) and is given by the Gaussian $\hat{v}(x)=\exp \left[\frac{1}{2}\left(1-x^{2}\right)\right]$. Thus $\hat{\mathcal{V}}$ is a singleton, and any centered ground state of $(*)$ converges to this Gaussian.

\subsection{Finite approximation of $(* *)$}

Lemma 16 below compares the variational problem $(* *)$ on $\mathbb{Z}^{d}$ with its restriction to $T_{N}=(-N, N]^{d} \cap \mathbb{Z}^{d}$ (with periodic boundary conditions). Recall Section 0.4 . Let $I, J$ be the functionals on $\mathcal{P}\left(\mathbb{Z}^{d}\right)$ defined in $(0.16-0.17)$. Let $I^{N}, J^{N}$ be their analogues on $\mathcal{P}\left(T_{N}\right)$. Put $F=I+\rho J$ and $F^{N}=I^{N}+\rho J^{N}$. Write $\mathcal{E}: \mathcal{P}\left(T_{N}\right) \rightarrow \mathcal{P}\left(\mathbb{Z}^{d}\right)$ to denote the canonical embedding defined by $\mathcal{E} p=p$ on $T_{N}$ and $\mathcal{E} p=0$ on $\mathbb{Z}^{d} \backslash T_{N}$.

Let $\mathcal{M}^{N} \subseteq \mathcal{P}\left(T_{N}\right)$ and $\mathcal{M} \subseteq \mathcal{P}\left(\mathbb{Z}^{d}\right)$ be the sets of minimizers of $F^{N}$ rsp. $F$. By compactness, $\mathcal{M}^{N}$ is non-empty. By assumptions $\mathrm{A} 1-\mathrm{A} 2$ in Theorem $1, \mathcal{M}$ is non-empty and is a singleton modulo shifts. In the following we shall write $\bar{p}^{N}$ to denote an arbitrary centered element of $\mathcal{M}^{N}$ and $\bar{p}$ to denote the unique centered element of $\mathcal{M}$. Let $\mathcal{U}_{\epsilon}^{N}, \mathcal{U}_{\epsilon}$ be the $\epsilon$-neighborhoods of $\mathcal{M}^{N}, \mathcal{M}$ in the $\ell^{1}$-metric. Define

$$
\begin{aligned}
\chi_{\epsilon}^{N}(\rho) & =\min _{p^{N} \notin \mathcal{U}_{\epsilon}^{N}} F^{N}\left(p^{N}\right) \\
\chi_{\epsilon}(\rho) & =\inf _{p \notin \mathcal{U}_{\epsilon}} F(p)
\end{aligned}
$$

and write $\chi^{N}(\rho), \chi(\rho)$ when $\epsilon=0$.

Lemma 16 Fix $\rho \in(0, \infty)$.

(a) $\lim _{N \rightarrow \infty} \chi^{N}(\rho)=\chi(\rho)$.

(b) $\lim _{N \rightarrow \infty}\left\|\mathcal{E} \bar{p}^{N}-\bar{p}\right\|_{\ell^{1}}=0$ for any $\left(\bar{p}^{N}\right)_{N \geq 1}$.

(c) $\mathcal{E U}_{\epsilon^{\prime}}^{N} \subseteq \mathcal{U}_{\epsilon}$ for all $0 \leq \epsilon^{\prime}<\epsilon$ and $N \geq N_{0}\left(\epsilon-\epsilon^{\prime}\right)$.

(d) $\mathcal{E}\left[\mathcal{U}_{\epsilon^{\prime \prime}}^{N}\right]^{c} \subseteq\left[\mathcal{U}_{\epsilon}\right]^{c}$ for all $0 \leq \epsilon<\epsilon^{\prime \prime}$ and $N \geq N_{0}\left(\epsilon^{\prime \prime}-\epsilon\right)$.

(e) $\lim \sup _{N \rightarrow \infty} \chi_{\epsilon^{\prime}}^{N}(\rho) \leq \chi_{\epsilon}(\rho)$ for all $0 \leq \epsilon^{\prime}<\epsilon$.

(f) $\liminf _{N \rightarrow \infty} \chi_{\epsilon^{\prime \prime}}^{N}(\rho) \geq \chi_{\epsilon}(\rho)$ for all $0 \leq \epsilon<\epsilon^{\prime \prime}$.

(g) $\chi_{\epsilon}(\rho)>\chi(\rho)$ for all $\epsilon>0$.

(h) For $p \in \mathcal{P}\left(\mathbb{Z}^{d}\right)$ and $S \subseteq \mathbb{Z}^{d}$, define $p(S)=\sum_{z \in S} p(z)$. Then for an arbitrary partition $\{A, B\}$ of $\mathbb{Z}^{d}$

$$
F(p) \geq \chi(\rho)-2 d p(\partial A \cup \partial B)-\rho[p(A) \log p(A)+p(B) \log p(B)] .
$$

Similarly on $T_{N}$ for any $N \geq 1$. 
Proof. Suppress $\rho$ from the notation.

(a) $\chi^{N} \leq \chi$ for all $N$ : For $p \in \mathcal{P}\left(\mathbb{Z}^{d}\right)$ let $\pi^{N} p \in \mathcal{P}\left(T_{N}\right)$ denote the periodization of $p$ w.r.t. $T_{N}$. Then $J^{N}\left(\pi^{N} p\right) \leq J(p)$ by concavity. Moreover, by the contraction principle,

$$
I^{N}\left(\pi^{N} p\right)=\inf _{q \in \mathcal{P}\left(\mathbb{Z}^{d}\right): \pi^{N} q=\pi^{N} p} I(q) .
$$

Hence

$$
\chi^{N}=\inf _{p \in \mathcal{P}\left(\mathbb{Z}^{d}\right)}\left[I^{N}\left(\pi^{N} p\right)+\rho J^{N}\left(\pi^{N} p\right)\right] \leq \inf _{p \in \mathcal{P}\left(\mathbb{Z}^{d}\right)}[I(p)+\rho J(p)]=\chi .
$$

$\underline{\liminf _{N \rightarrow \infty} \chi^{N} \geq \chi}$ : For all $p^{N} \in \mathcal{P}\left(T_{N}\right)$ we have

$$
\begin{aligned}
& 0 \leq I\left(\mathcal{E} p^{N}\right)-I^{N}\left(p^{N}\right) \leq d \sum_{z \in \partial T_{N}} p^{N}(z) \\
& J\left(\mathcal{E} p^{N}\right)=J^{N}\left(p^{N}\right),
\end{aligned}
$$

as is easily deduced from $(0.17-0.18)$. (The upper bound estimates the sum of $p(x)+p(y)$ over all $x, y$ connected by a bond that is 'cut open'.) Hence

$$
0 \leq F\left(\mathcal{E} p^{N}\right)-F^{N}\left(p^{N}\right) \leq d \sum_{z \in \partial T_{N}} p^{N}(z)
$$

We have proved in Section 5.1.1 that $\bar{p}$ is a product measure with all its marginals unimodal. The same argument works for $\bar{p}^{N}$ without modification. Thus we know, in particular, that

$$
\sum_{z \in \partial T_{N}} \bar{p}^{N}(z) \leq\left|\partial T_{N}\right| /\left|T_{N}\right|
$$

It therefore follows that

$$
\chi^{N}=F^{N}\left(\bar{p}^{N}\right) \geq F\left(\mathcal{E} \bar{p}^{N}\right)-d\left|\partial T_{N}\right| /\left|T_{N}\right| \geq \chi-d\left|\partial T_{N}\right| /\left|T_{N}\right| .
$$

Take the limit $N \rightarrow \infty$ to get the claim.

(b) The unimodality of $\bar{p}^{N}$ implies that $\left(\mathcal{E} \bar{p}^{N}\right)_{N \geq 1}$ is tight. Let $\left(N_{k}\right)$ be any subsequence such that $\mathcal{E} \bar{p}^{N_{k}} \rightarrow \tilde{p}$ in $\ell^{1}$ for some $\tilde{p} \in \mathcal{P}\left(\mathbb{Z}^{d}\right)$ as $\bar{k} \rightarrow \infty$. With the help of (5.92-5.93) and the lower semicontinuity of $F$, we get

$$
\liminf _{k \rightarrow \infty} F^{N_{k}}\left(\bar{p}^{N_{k}}\right)=\liminf _{k \rightarrow \infty} F\left(\mathcal{E} \bar{p}^{N_{k}}\right) \geq F(\hat{p}) .
$$

Since the 1.h.s. is $\chi$ by (a), it follows that $\tilde{p}$ is a minimizer of $F$. Hence $\tilde{p}=\bar{p}$, proving the claim.

(c) For $x \in \mathbb{Z}^{d}$, let $\theta_{x}: \mathcal{P}\left(\mathbb{Z}^{d}\right) \rightarrow \mathcal{P}\left(\mathbb{Z}^{d}\right)$ denote the $x$-shift defined by $\left(\theta_{x} p\right)(y)=p(x+y)$. For every $p \in \mathcal{P}\left(\mathbb{Z}^{d}\right)$ we have

$$
\left\|\theta_{x} p-\bar{p}\right\|_{\ell^{1}} \leq\left\|\theta_{x} p-\mathcal{E} \bar{p}^{N}\right\|_{\ell^{1}}+\left\|\mathcal{E} \bar{p}^{N}-\bar{p}\right\|_{\ell^{1}} .
$$

Take the infimum over $x$ on both sides to obtain that $p \notin \mathcal{U}_{\epsilon} \Longrightarrow p \notin \mathcal{E} \mathcal{U}_{\epsilon-\delta_{N}}^{N}$ with $\delta_{N}=\left\|\mathcal{E} \bar{p}^{N}-\bar{p}\right\|_{\ell^{1}}$. The claim now follows from (b). 
(d) For $x \in \mathbb{Z}^{d}$, let $\theta_{x}^{N}: \mathcal{P}\left(T_{N}\right) \rightarrow \mathcal{P}\left(T_{N}\right)$ and $\hat{\theta}_{x}^{N}: \mathcal{P}\left(\mathbb{Z}^{d}\right) \rightarrow \mathcal{P}\left(\mathbb{Z}^{d}\right)$ denote the $N$-periodic $x$-shifts defined by

$$
\begin{aligned}
& \left(\theta_{x}^{N} p^{N}\right)(y)=p^{N}\left(x+y\left(\bmod T_{N}\right)\right) \\
& \left(\hat{\theta}_{x}^{N} p\right)(y)= \begin{cases}p\left(x+y\left(\bmod T_{N}\right)\right) & y \in T_{N} \\
p(y) & y \in \mathbb{Z}^{d} \backslash T_{N}\end{cases}
\end{aligned}
$$

We obviously have

$$
\mathcal{E} \cdot \theta_{x}^{N}=\hat{\theta}_{x}^{N} \cdot \mathcal{E} \text { on } \mathcal{P}\left(T_{N}\right)
$$

Moreover, it is easy to see that for any $x \in \mathbb{Z}^{d}$ and for any $p, q \in \mathcal{P}\left(\mathbb{Z}^{d}\right)$ with support in $T_{N}$

$$
\left\|\theta_{x} p-q\right\|_{\ell^{1}} \geq\left\|\hat{\theta}_{x}^{N} p-q\right\|_{\ell^{1}} .
$$

Combining (5.98-5.99), we get that for any $p^{N} \in \mathcal{P}\left(T_{N}\right)$

$$
\left\|\theta_{x} \mathcal{E} p^{N}-\mathcal{E} \bar{p}^{N}\right\|_{\ell^{1}} \geq\left\|\hat{\theta}_{x}^{N} \mathcal{E} p^{N}-\mathcal{E} \bar{p}^{N}\right\|_{\ell^{1}}=\left\|\mathcal{E} \theta_{x}^{N} p^{N}-\mathcal{E} \bar{p}^{N}\right\|_{\ell^{1}}
$$

and hence

$$
\left\|\theta_{x} \mathcal{E} p^{N}-\bar{p}\right\|_{\ell^{1}} \geq\left\|\mathcal{E} \theta_{x}^{N} p^{N}-\mathcal{E} \bar{p}^{N}\right\|_{\ell^{1}}-\delta_{N}
$$

with $\delta_{N}=\left\|\mathcal{E} \bar{p}^{N}-\bar{p}\right\|_{\ell^{1}}$. Take the infimum over $x$ on both sides to obtain that $\mathcal{E} p^{N} \in$ $\mathcal{E}\left[\mathcal{U}_{\epsilon^{\prime \prime}}^{N}\right]^{c} \Longrightarrow \mathcal{E} p^{N} \in\left[\mathcal{U}_{\epsilon^{\prime \prime}-\delta_{N}}\right]^{c}$. The claim now follows from (b).

(e) From (c) and the inequality $F^{N}\left(p^{N}\right) \leq F\left(\mathcal{E} p^{N}\right)$ (recall (5.92)) we get

$$
\chi_{\epsilon^{\prime}}^{N}=\min _{p^{N} \notin \mathcal{U}_{\epsilon^{\prime}}^{N}} F^{N}\left(p^{N}\right) \leq \min _{p^{N} \notin \mathcal{U}_{\epsilon^{\prime}}^{N}} F\left(\mathcal{E} p^{N}\right) \leq \inf _{p \notin \mathcal{U}_{\epsilon}} F(p)=\chi_{\epsilon} .
$$

(f) Let $\bar{p}_{\epsilon^{\prime \prime}}^{N}$ denote an arbitrary centered minimizer for $\chi_{\epsilon^{\prime \prime}}^{N}=\min _{p^{N} \notin \mathcal{U}_{\epsilon \prime \prime}^{N}} F^{N}\left(p^{N}\right.$ ) (which exists by compactness). Then there exists some $y=y\left(\bar{p}_{\epsilon^{\prime \prime}}^{N}\right) \in T_{N}$ such that

$$
\sum_{z \in \partial T_{N}}\left(\theta_{y}^{N} \bar{p}_{\epsilon^{\prime \prime}}^{N}\right)(z) \leq\left|\partial T_{N}\right| /\left|T_{N}\right|
$$

and hence

$$
\chi_{\epsilon^{\prime \prime}}^{N}=F^{N}\left(\bar{p}_{\epsilon^{\prime \prime}}^{N}\right)=F^{N}\left(\theta_{y}^{N} \bar{p}_{\epsilon^{\prime \prime}}^{N}\right) \geq F\left(\mathcal{E} \theta_{y}^{N} \bar{p}_{\epsilon^{\prime \prime}}^{N}\right)-d\left|\partial T_{N}\right| /\left|T_{N}\right|
$$

(compare with (5.93-5.94)). Because $\theta_{y}^{N} \bar{p}_{\epsilon^{\prime \prime}}^{N} \notin \mathcal{U}_{\epsilon^{\prime \prime}}^{N}$, it follows from (d) that for $N$ sufficiently large

$$
F\left(\mathcal{E} \theta_{y}^{N} \bar{p}_{\epsilon^{\prime \prime}}^{N}\right) \geq \chi_{\epsilon} .
$$

Now combine (5.104-5.105) and let $N \rightarrow \infty$, to get the claim.

(g) We shall need the following property, which will be proved at the end:

$$
\text { Any centered minimizing sequence for } \chi=\min _{p \in \mathcal{P}\left(\mathbb{Z}^{d}\right)} F(p) \text { is tight. }
$$


Suppose that $\chi=\chi_{\epsilon}$ for some $\epsilon>0$. Let $\left(p_{\epsilon, n}\right)$ be any centered minimizing sequence for (5.87). Then, by (5.106), this sequence is tight. Hence $p_{\epsilon, n}$ converges to some $p_{\epsilon} \notin \mathcal{U}_{\epsilon}$ along some subsequence. Because $F$ is lower semicontinuous, it follows that $\chi=\chi_{\epsilon} \geq F\left(p_{\epsilon}\right)$. But this in turn implies that $p_{\epsilon}$ is some shift of $\bar{p}$, which contradicts $p_{\epsilon} \notin \mathcal{U}_{\epsilon}$. Thus we must have $\chi_{\epsilon}>\chi$ for all $\epsilon>0$, as claimed.

It remains to prove (5.106). Let $\left(p_{n}\right)$ be any centered sequence that is not tight. Then there exist sequences $\left(n_{k}\right),\left(N_{k}\right)$ and some $\delta>0$ such that

$$
\begin{array}{ll}
\sum_{z \in T_{N_{k}}} p_{n_{k}}(z) & =a_{k} \geq \delta \quad \text { for all } k \\
\sum_{z \in \mathbb{Z}^{d} \backslash T_{N_{k}}} p_{n_{k}}(z) & =b_{k} \geq \delta \quad \text { for all } k \\
\sum_{z \in \partial T_{N_{k}} \cup \partial\left(\mathbb{Z}^{d} \backslash T_{N_{k}}\right)} p_{n_{k}}(z) & =c_{k} \rightarrow 0 \quad \text { as } k \rightarrow \infty .
\end{array}
$$

Define

$$
\begin{aligned}
& p_{k}^{\prime}=\frac{1}{a_{k}} p_{n_{k}} 1_{T_{N_{k}}} \\
& p_{k}^{\prime \prime}=\frac{1}{b_{k}} p_{n_{k}} 1_{\mathbb{Z}^{d} \backslash T_{N_{k}}} .
\end{aligned}
$$

Then we have (compare with (5.91))

$$
\begin{aligned}
& I\left(p_{n_{k}}^{\prime}\right)=I\left(a_{k} p_{k}^{\prime}+b_{k} p_{k}^{\prime \prime}\right) \geq a_{k} I\left(p_{k}^{\prime}\right)+b_{k} I\left(p_{k}^{\prime \prime}\right)-d c_{k} \\
& J\left(p_{n_{k}}^{\prime}\right)=J\left(a_{k} p_{k}^{\prime}+b_{k} p_{k}^{\prime \prime}\right)=a_{k} J\left(p_{k}^{\prime}\right)+b_{k} J\left(p_{k}^{\prime \prime}\right)-a_{k} \log a_{k}-b_{k} \log b_{k} .
\end{aligned}
$$

Hence

$$
\begin{aligned}
F\left(p_{n_{k}}\right) & =I\left(p_{n_{k}}^{\prime}\right)+\rho J\left(p_{n_{k}}^{\prime}\right) \\
& \geq a_{k} F\left(p_{k}^{\prime}\right)+b_{k} F\left(p_{k}^{\prime \prime}\right)-d c_{k}-\rho\left[a_{k} \log a_{k}+b_{k} \log b_{k}\right] \\
& \geq \chi-d c_{k}-\rho\left[a_{k} \log a_{k}+b_{k} \log b_{k}\right]
\end{aligned}
$$

$\left(a_{k}+b_{k}=1\right)$. But $c_{k} \rightarrow 0$ and both $a_{k}$ and $b_{k}$ are bounded away from 0 and 1 . Therefore $\liminf \inf _{k \rightarrow \infty} F\left(p_{n_{k}}\right)>\chi$, and so we conclude that $\left(p_{n}\right)$ is not minimizing.

(h) Same as the argument in (5.107-5.110).

Acknowledgment The authors thank E. Bolthausen and J.-D. Deuschel for discussions on some of the technical points in Section 3. 


\section{References}

[1] P.W. Anderson, Absence of diffusion in certain random lattices. Phys. Rev. 109 (1958) 1492-1505.

[2] P. Antal, Enlargement of obstacles for the simple random walk. Ann. Probab. 23 (1995) 1061-1101.

[3] E. Bolthausen and U. Schmock, On self-attracting $d$-dimensional random walks. Preprint 1994. To appear in Ann. Probab.

[4] R.A. Carmona and S.A. Molchanov, Parabolic Anderson Problem and Intermittency, AMS Memoir 518, American Mathematical Society, Providence RI 1994.

[5] D.A. Dawson and G. Ivanoff, Branching diffusions and random measures. Adv. Probab. Relat. Top. 5 (1978) 61-104.

[6] J.-D. Deuschel and D.W. Stroock, Large Deviations, Academic Press, Boston 1989.

[7] W. Ebeling, A. Engel, B. Esser and R. Feistel, Diffusion and reaction in random media and models of evolution processes. J. Stat. Phys. 37 (1984) 369-385.

[8] J. Fröhlich, F. Martinelli, E. Scoppola and T. Spencer, Constructive proof of localization in the Anderson tight binding model. Commun. Math. Phys. 101 (1985) 21-46.

[9] J. Gärtner and S.A. Molchanov, Parabolic problems for the Anderson Hamiltonian. I. Intermittency and related topics. Commun. Math. Phys. 132 (1990) 613-655.

[10] J. Gärtner and S.A. Molchanov, Parabolic problems for the Anderson Hamiltonian. II. Structure of high peaks and Lifshitz tails. Preprint 1996.

[11] A. Greven and F. den Hollander, Branching random walk in random environment: phase transitions for local and global growth rates, Probab. Theory Relat. Fields 91 (1992) 195-249.

[12] A.-S. Sznitman, Brownian motion with a drift in a Poissonian potential. Comm. Pure Appl. Math. 47 (1994) 1283-1318.

[13] Ya.B. Zel'dovich, Selected Papers. Chemical Physics and Hydrodynamics (in russian), Nauka, Moscow 1984. 